University of Louisville

ThinkIR: The University of Louisville's Institutional Repository

8-2014

\title{
Numerical investigation of hydrodynamic focusing and coulter principle in a microfluidic device
}

Muheng Zhang

University of Louisville

Follow this and additional works at: https://ir.library.louisville.edu/etd

Part of the Mechanical Engineering Commons

\section{Recommended Citation}

Zhang, Muheng, "Numerical investigation of hydrodynamic focusing and coulter principle in a microfluidic device" (2014). Electronic Theses and Dissertations. Paper 1644.

https://doi.org/10.18297/etd/1644

This Doctoral Dissertation is brought to you for free and open access by ThinkIR: The University of Louisville's Institutional Repository. It has been accepted for inclusion in Electronic Theses and Dissertations by an authorized administrator of ThinkIR: The University of Louisville's Institutional Repository. This title appears here courtesy of the author, who has retained all other copyrights. For more information, please contact thinkir@louisville.edu. 


\title{
NUMERICAL INVESTIGATION OF
}

\section{HYDRODYNAMIC FOCUSING AND COULTER PRINCIPLE IN A MICROFLUIDIC DEVICE}

\author{
By Muheng Zhang \\ A Dissertation \\ Submitted to the Faculty of the \\ in Partial Fulfillment of the Requirements \\ for the Degree of \\ Doctor of Philosophy \\ Department of Mechanical Engineering \\ University of Louisville \\ Louisville, Kentucky
}

J. B. Speed School of Engineering of the University of Louisville

August 2014 



\title{
NUMERICAL INVESTIGATION OF
}

\section{HYDRODYNAMIC FOCUSING AND COULTER PRINCIPLE IN A MICROFLUIDIC DEVICE}

\author{
By Muheng Zhang \\ A Dissertation Approved on
}

April 232014

by the following Dissertation Committee:

Dr. Yongsheng Lian: Dissertation Director

Dr. Ellen G Brehob

Dr. Cindy Harnett

Dr. Michael K Sharp

Dr. Stuart Williams 


\section{ACKNOWLEDGMENTS}

I would like to thank Professor Yongsheng Lian for giving me this opportunity to research in such a fantastic area. His perseverance, knowledge and enthusiasm made me to become independent and optimistic to face the problems. I would also like to thanks

my advisor Dr. Brehob, Dr. Williams, Dr. Harnett and Dr. Sharp for their supports and many stimulating suggestions.

I would also like to acknowledge funding from the Department of Mechanical Engineering and Speed School. A special acknowledgement goes to Dr. Chaolei Zhang who I owe my deepest respect for helping me the basics of the numerical analysis and simulation software; I am also thankful to the people who helped me a lot during my lab work: Timothy M Broering, Kyle Hord, Guibo Li, Haishui Jin, Guojie Song, Dongsheng Yang, Yan Chen, Russel Prater, Han Li, Andrew Work, Stephen Zwanzig, Matt Fuller and Kyle Schmidt. 


\section{ABSTRACT \\ NUMERICALINVESTIGATION OF HYDRODYNAMIC \\ FOCUSING AND COULTER PRINCIPLE IN A \\ MICROFLUIDIC DEVICE}

\section{Muheng Zhang}

\section{April 23, 2014}

Particle information such as particle size and amount is significant to many fields such as chemical engineering, biological research, medical analysis, and environment detection. Microfluidic Coulter counter is suitable for this task because it can get fast and accurate analysis. However, the fundamental principle and several design strategies details are still needed to be analyzed and modeled in order to improve its performance.

This dissertation focused on several challenges from the numerical simulation aspects. First one is the analysis for the hydrodynamic focusing in microfluidic Coulter Counter. In recent years, many microfluidic Coulter Counter designs have utilized a technique termed "hydrodynamic focusing" to control the particle's trajectory. 
Hydrodynamic focusing uses two sheath flows (low concentration) to squeeze the sample flow (high concentration) so it can concentrate the ion distribution on the region where the designer wants. There are large concentration difference between the sample stream and sheath stream. However, few published papers mentioned the ion concentration distribution under the impacts of hydrodynamic focusing. This is difficult to deal withbecause there are conductivity differences at the interface of the sheath/sample streams and the interface of the sample stream/particle. A very fine grid mesh is needed to capture the ion concentration distribution. Meanwhile the simulation which runs at low Reynolds number requires a rigorously smaller than usual time interval to satisfy the stability condition. Another challenge which this dissertation faced is the particle motion. The key point for particle motion simulation is the grid regeneration since the grid needs to be adaptive with the particle movement. So far many simulation solvers choose to regenerate the whole grid domain after each time step. This strategy takes a huge amount of time. The third challenge is the simulation for the electrical potential by considering the significant conductivity difference between the particle and medium. The numerical simulation process gets unstable when the conductivity distribution is not uniform and becomes worse if there is a sharp conductivity variation in the distribution. A new mathematical difference method is needed to model the potential distribution.

In order to overcome these challenges, this dissertation used an "overlap mapping" grid generation method to capture the ion concentration distribution along the interfaces of sheath/sample stream and sample stream/particle. The grid regeneration was only 
needed for the moving object in order to save computing time. A new center difference method was applied on the electrical potential equation to solve its stability problem.

The numerical simulation studied the impacts of hydrodynamic focusing with considering the ion convection-diffusion phenomenon. We found that the fluid viscosity does not play a significant role in the concentration distribution. The max concentration relative error is no more than $2.5 \%$ on the peak of the selected cross section. Also, the diffusion phenomenon can dilute the ion concentration focusing ability by hydrodynamic focusing while larger sample/sheath stream flow rates ratio can improve the concentration value. The hydrodynamic focusing can decrease the vertical gap between the particles while increasing their horizontal gap. After that the current signal variation were simulated and it showed that the diffusion phenomenon can decrease the signal sensitivity. For the particle we choose to simulate, the current sensitivity decreases $29 \%$ in $2 \mathrm{D}$ simulation. Fluid viscosity does not play a significant role in the current signal variation if we choose the Reynolds number between one and ten (The max current variation is $3.6 \%$ for the $3 \mathrm{~d}$ simulation). The electrodes length/position, particle size and particle position also affected the current signal. The numerical simulation approaches and results from this dissertation updated the understanding of hydrodynamic focusing and Coulter Counter principle. Also this dissertation showed the feasibility to study the Coulter counter principle numerically by considering the diffusion phenomenon and particle motion. 


\section{TABLE OF CONTENTS}

\section{PAGE}

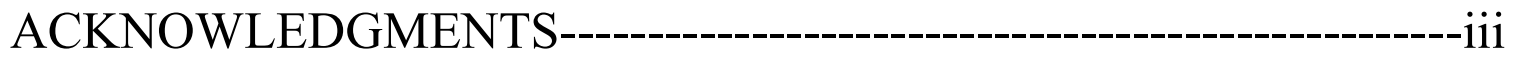

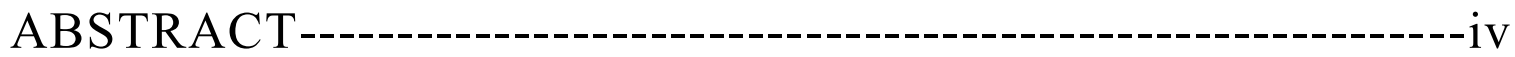

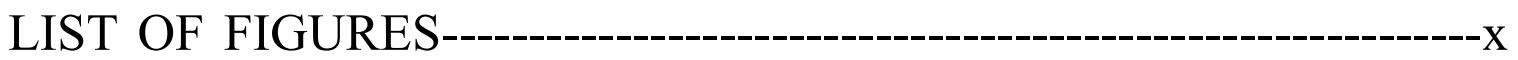

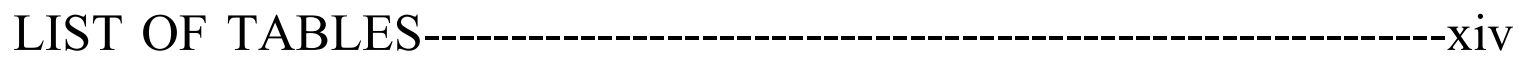

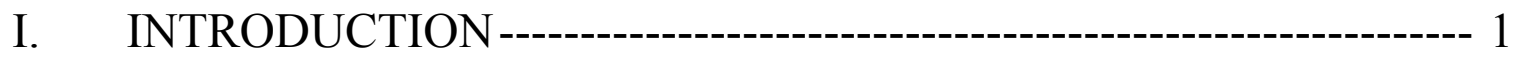

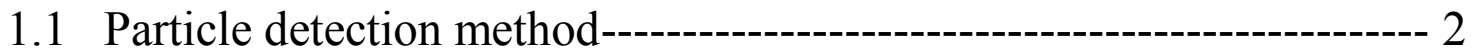

1.2 Previous work of microfluidic Coulter Counter--------------------- 8

1.3 Challenges in the numerical study of Coulter Counter ---------------28

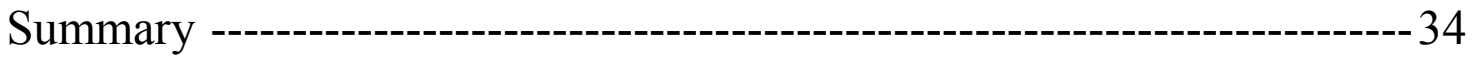

II. EQUATIONS AND NUMERICAL METHODS -------------------- 35

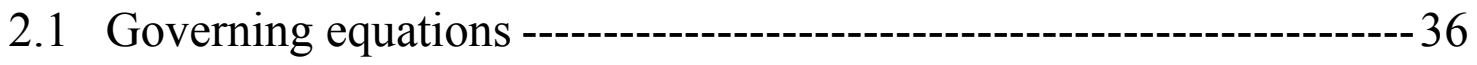

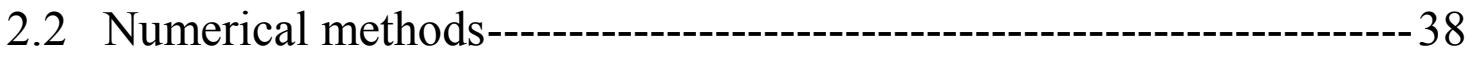

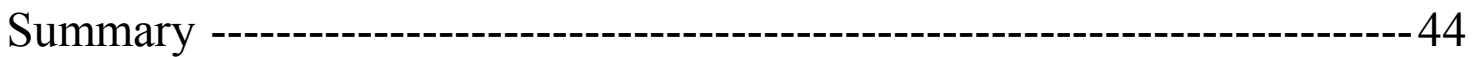

III. CODE VALIDATION -- 45

3.1 Steady, laminar flow between infinite fixed parallel plates-----------46

3.2 Simulation of hydrodynamic focusing------------------------48

3.3 Velocity and ion concentration distribution on 3D micro channel --50 


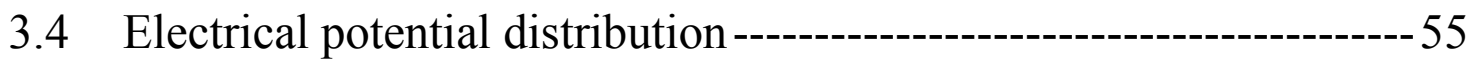

Summary --------------------------------------------------------------------------------60

IV. SIMULATION FOR HYDRODYNAMIC FOCUSING -------------- 61

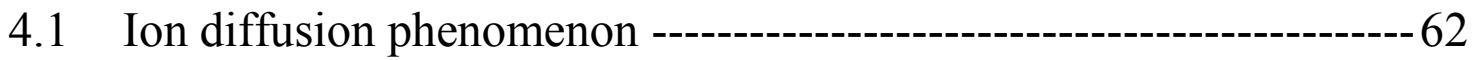

4.2 The impacts of Reynolds number -----------------------------------63

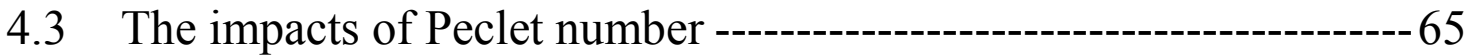

4.4 The impacts of relative sample and sheath flow rate -----------------66

4.5 Three dimensional simulation for the hydrodynamic focusing-------71

4.6 Particle motion study --------------------------------------------------------- 83

4.7 Three-inlets/Three outlets micro channel study ----------------------- 88

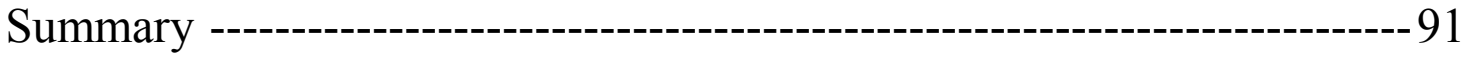

V. TWO DIMENSIONAL SIMULATION FOR COULTER

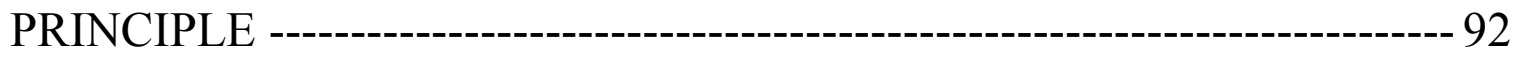

5.1 The impacts of molecular diffusion phenomenon--------------------93

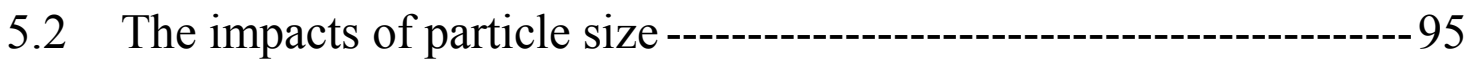

5.3 The impacts of electrode length and gap between the electrodes ---96

5.4 The impacts of electrode position ----------------------------------97

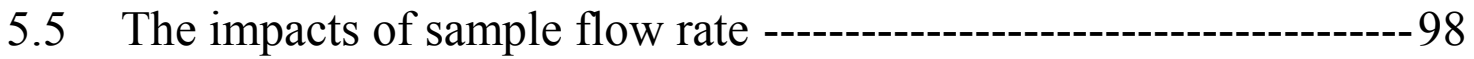

5.6 The impacts of parallel electrodes distribution ------------------------99

Summary --------------------------------------------------------------------------- 101 


\section{THREE DIMENSIONAL SIMULATION FOR COULTER}

PRINCIPLE ----------------------------------------------------------------------- 102

6.1 The schematic of the simulation domain ---------------------------- 103

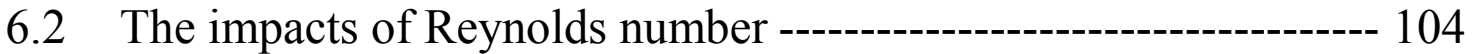

6.3 The impacts of the Peclet number----------------------------------- 105

6.4 The impacts of the particle size------------------------------------ 107

6.5 The impacts of the particle position------------------------------ 107

6.6 The impacts of the sample stream flow rate -------------------- 108

6.7 The impacts of the electrodes length/gap --------------------------- 109

6.8 The impacts of the electrodes position -------------------------- 110

6.9 The impacts of parallel electrodes distribution -------------------- 112

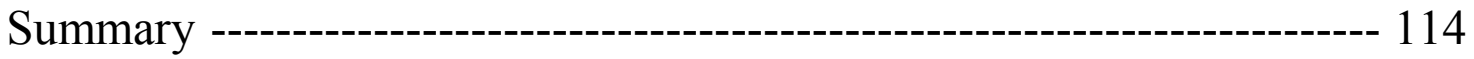

VII. CONCLUSION AND FUTURE WORK ---------------------------- 115

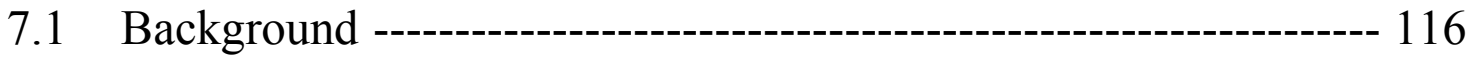

7.2 Governing equation and numerical method -------------------------- 116

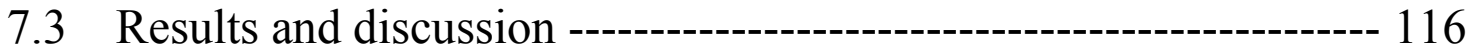

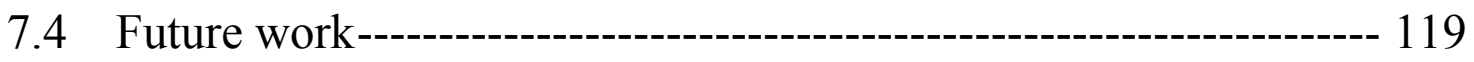

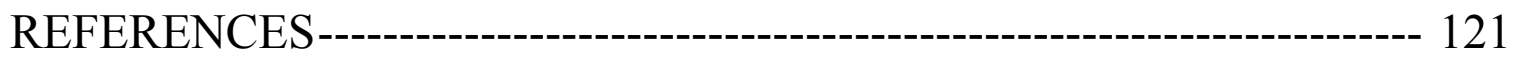

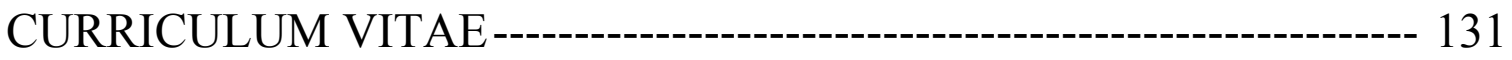




\section{LIST OF FIGURES}

Figure I-1 Microfluidic particle detection chip (Saleh and Sohn (2002)). ....................... 2

Figure I-2 Schematic representation light scattering (eventsHerzenberg et al. (2002)).... 3

Figure I-3 Light-blocking technique (Zhang et al. (2009))............................................ 4

Figure I-4 Basic schematic of a traditional Coulter Counter. ........................................ 5

Figure I-5 The simplified electrical model for Coulter Counter..................................... 6

Figure I-6 The front view of a multi-aperture Coulter Counter (Ashish (2006)).............. 8

Figure I-7 Hydrodynamic focusing for detection (Wang et al. (2005))......................... 10

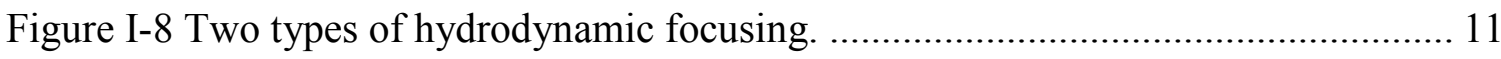

Figure I-9 Designs of 2D/3D hydrodynamic focusing (Scott et al. (2008a)). ................. 12

Figure I-10 Chevron-based sheath flow design. (Howell et al. (2008)) ......................... 13

Figure I-11 3D hydrodynamic focusing with one sheath stream (Lee et al. (2009))....... 13

Figure I-12 The concentration distribution function $(\mathrm{Wu}(2005))$................................ 15

Figure I-13 The asymmetric hydrodynamic focusing (Lee (2006)). ............................ 17

Figure I-14 Distribution of the focused sample stream for three different Reynolds

number and four different focusing ratios (Blonski et al. (2011)) ................................ 18

Figure I-15 Focused sample stream width considering the Reynolds number variation

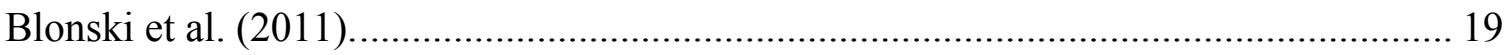

Figure I-16 The sample stream is focused by three sheath flows (Nieuwenhuis et al., 2004).

Figure I-17 The schematic for electrical detection method configuration under the

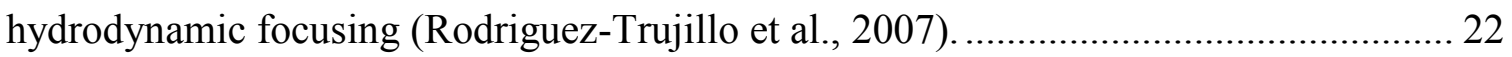

Figure I-18 A microfluidic Coulter Counter by choosing oil as the sheath stream

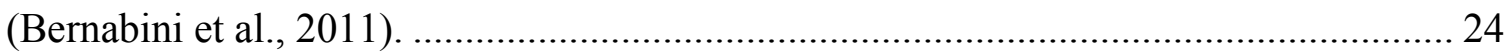

Figure I-19 The schematic of the test points by marking with red circles..................... 31 
Figure I-20 Simulation potential fields with three different conductivities of the tested points

Figure II-1 The correlation between the electrical conductivity and the ion concentration.

Figure II-2 The current integration through certain cross section. ............................... 38

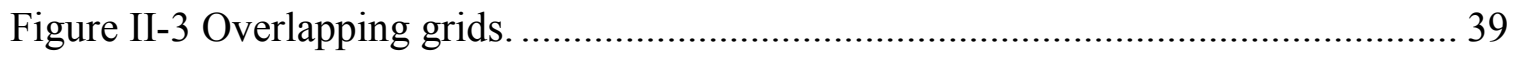

Figure III-1 The schematic of the channel and its dimensions. ................................... 46

Figure III-2 Comparison of downstream velocity distribution at the fully developed

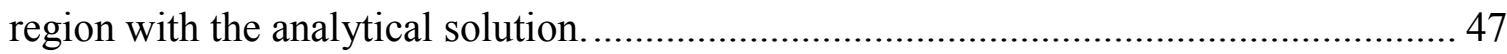

Figure III-3 Concentration for a sample stream injected into a 2D channel................... 48

Figure III-4 Simulation results of ion concentration distribution at different downstream locations.

Figure III-5 The simulation domain of the three dimensional micro channel. ................ 50

Figure III-6 Analytical solution about the velocity distribution and simulation results... 52 Figure III-7 Ion concentration distribution. Left one is the mesh (half of the domain) by Comsol, right one is the simulation results including Overture and Comsol. .................. 52

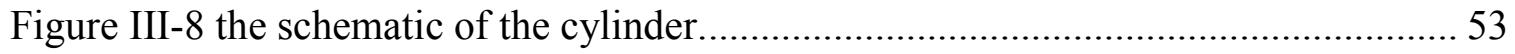

Figure III-9 Comparison with the analytical and numerical solution for the convection only situation.

Figure III-10 Schematic of the one-dimensional conductive bars. Top: one-dimensional bar with uniform electrical conductivity; bottom: one-dimensional bar with non-uniform conductivity, the red region has low conductivity. $V(x)$ is the potential distribution along the one dimensional bar. 56 Figure III-11 Comparison of electric potential distribution along a bar with different conductivities. Top one is the case with uniform conductivity, bottom one is the case with non-uniform conductivity. 56 Figure III-12 The coplanar electrodes distribution and SCM transfer mapping (Sun et al.(2007)) 57

Figure III-13 Schematic of a finite length channel with electrodes mounted on the bottom. 
Figure III-14 Comparison of the electrical magnitude between numerical and analytical

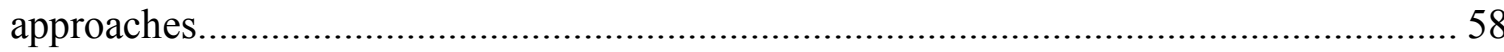

Figure III-15 Potential distribution in a 2D channel................................................... 59

Figure IV-1 Sample flow concentration due to hydrodynamic focusing......................... 62

Figure IV-2 The concentration profiles at different locations. .............................................6 63

Figure IV-3 The concentration profiles at different Reynolds numbers by fixing Peclet number.

Figure IV-4 The concentration profiles at different Reynolds numbers by fixing Schmidt number. 65

Figure IV-5 The concentration profiles at different $\mathrm{Pe}$ with $\mathrm{Re}=1$............................... 66

Figure IV-6 Concentration along the center line for varied relative flow rates................ 67

Figure IV-7 Concentration profiles for varied relative flow rate..................................... 68

Figure IV-8 The micro channel with the mark of interface.............................................. 69

Figure IV-9 Concentration distribution with different sheath flow rates differences....... 70

Figure IV-10 Ion concentration distribution for hydrodynamic focusing. ....................... 72

Figure IV-11 Contours of ion concentration distribution at different downstream positions

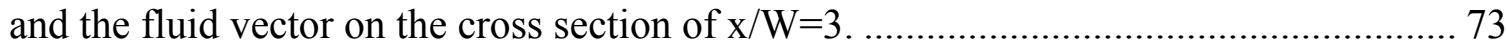

Figure IV-12 Lines of the ion concentration distribution at different positions............... 74 Figure IV-13 Cross sections of ion concentration distribution at the different $Z$ positions.

Figure IV-14 The concentration profiles at different Reynolds numbers by fixing the Peclet number.

Figure IV-15 The concentration profiles at different Reynolds numbers by fixing the

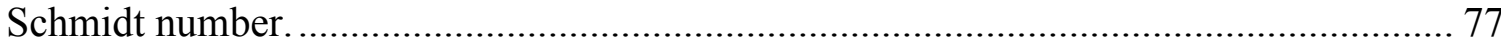

Figure IV-16 Concentration along the center line for varied Peclet number.................... 78

Figure IV-17 Impacts of the Peclet number variation. ................................................. 78

Figure IV-18 Concentration along the center line for varied relative flow rates.............. 79

Figure IV-19 Concentration along the fixed line for varied relative flow rates. .............. 79

Figure IV-20 ion concentration distribution under the different asymmetric situation.... 80 
Figure IV-21 Definition of the concentration peak bias under the asymmetry phenomenon.

Figure IV-22 Correlation between the concentration peak bias and sheath stream flow rates difference.

Figure IV-23 Correlation between the concentration peak bias and sample stream flow rates.

Figure IV-24 The correlation between the concentration peak bias and micro channel

height.

Figure IV-25 The motion of a particle under hydrodynamic focusing (single particle)... 84

Figure IV-26 The grids for the simulation of two particles in a micro channel. ............. 84

Figure IV-27 Snapshots of the particle motion in a micro-channel............................... 85

Figure IV-28 Distance between the two particles as a function of time......................... 86

Figure IV-29 multiple particles moving in the micro channel................................... 86

Figure IV-30 Snapshots of the particle motion in a micro-channel by 3D simulation..... 87

Figure IV-31 Distance between the two particles as a function of time by 3D simulation.

Figure IV-32 The streamlines in a three-inlet/three-outlet micro-channel device.

Figure IV-33 Concentration contours with different sample stream flow rates.

Figure V-1 Conductivity distribution in a micro fluid Coulter counter. Sheath flows are injected from top/bottom inlets and conductive flow is injected from middle inlet.

Figure V-2 Comparison of conductivity distribution of sample flows with and without molecular diffusivity at $\mathrm{x} / \mathrm{W}=7$

Figure V-3 Comparison of current sensitivity due to the presence of a low-conductivity particle with and without molecular diffusivity.

Figure V-4 Impact of particle size on the current sensitivity. A larger particle results in higher sensitivity.

Figure V-5 Variation of current sensitivity with electrode length and gap. Top one is the impact of electrodes gap, bottom one is the impact of electrodes length. 96

Figure V-6 Three different electrode locations along the channel. 97 
Figure V-7 Cross sectional conductivity distribution at the centers of region A, B and C.

Figure V-8 Impact of electrode position on the current sensitivity. ............................. 98

Figure V-9 Impact of the sheath stream flow rate on the current sensitivity.................. 99

Figure V-10 Electric potential distribution under the parallel electrodes distribution. .... 99

Figure V-11 Current sensitivity under the parallel electrodes distribution................... 100

Figure VI-1 The three dimensional simulation domain........................................... 103

Figure VI-2 The size of the simulation and the electrodes configuration...................... 104

Figure VI-3 Impact of Reynolds number from one to ten on the current sensitivity...... 105

Figure VI-4 Impact of particle size on the current sensitivity. A larger particle results in

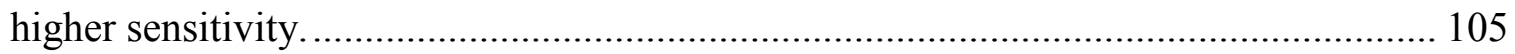

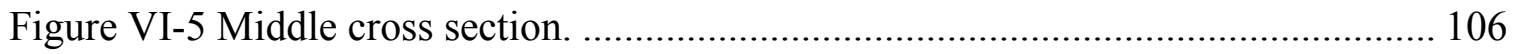

Figure VI-6 Current density distribution on the middle cross section........................ 106

Figure VI-7 Impact of particle size on the current sensitivity. .................................. 107

Figure VI-8 Impact of particle height on the current sensitivity. ............................... 108

Figure VI-9 Impact of sample stream flow rates on the current sensitivity.................. 109

Figure VI-10 Impact of electrodes length and gap between the electrodes.................. 110

Figure VI-11 Three different positions of simulation domain.................................. 111

Figure VI-12 Impact of electrodes position translation. ....................................... 112

Figure VI-13 Two different electrode distributions.............................................. 112

Figure VI-14 The comparison of two different electrodes distributions. ..................... 113 


\section{LIST OF TABLES}

Table I-1 Several particle detection methods................................................................ 7

Table I-2 Research situation for aperture design. .................................................. 10

Table I-3 Research situation for hydrodynamic focusing from experiment aspect......... 14

Table I-4 Research for hydrodynamic focusing from analytical/numerical aspects........ 21

Table I-5 Published papers about the electrical field configuration and signal variation. 23

Table I-6 Published papers about the signal variation from analytical/simulation aspects.

Table III-1 Grid sensitivity test for the single channel. ................................................... 46

Table III-2 Relative max error in concentration using different grid spacing. ................ 49

Table III-3 Two different grid density cases............................................................. 51

Table IV-1 Comparison of displacement from analytical and numerical simulations. .... 71 


\section{INTRODUCTION}

This chapter first has a brief introduction of the different kinds of particle detection techniques. Then the Coulter Counter principle is introduced. Following that is a detailed literature review including typical experimental design, theoretical development, and numerical simulations results. After that the numerical simulation challenges for micro Coulter Counter are introduced. Finally, the objectives of this dissertation are stated. 


\subsection{Particle detection method}

Particle detection is a very useful technique which is widely used in chemistry, biology and medicine (Ito et al. (2004), Tan et al. (2003), Rawstron et al. (2013)). Due to the huge progress of micro fabrication in the recent two decades, the microfluidic particle detection devices have shown excellent performance in many aspects such as detection speed, detection accuracy and detection range. Figure I-1 shows one typical microfluidic device that can be used to detect the existence of particles. The two reservoirs are connected by a small pore and it is fabricated by sealing PDMS to glass. Particles in the reservoirs are pushed through the tiny pore, partially blocking the ion motion when the electrical field is applied in the device.

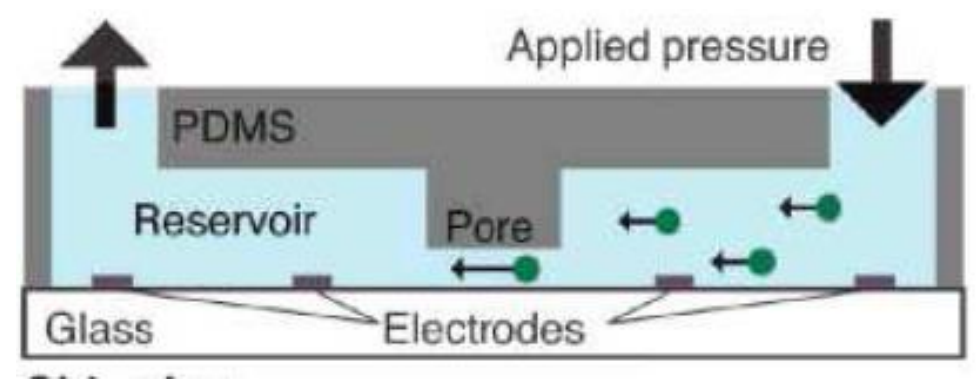

Figure I-1 Microfluidic particle detection chip (Saleh and Sohn (2002)).

Several methods are used to detect the particle size and position based on the application of the device. One is called light scattering. As shown in Figure I-2, when light shines on a particle that is transparent, the transmitted light coming out of the particle can be divided into two types. One is at a small angle with the light emission direction (between $0.5^{\circ}$ and $5^{\circ}$ ) which is called Forward Scattered (FSC); the other one is at a large angle with the light source direction (between $15^{\circ}$ and $150^{\circ}$ ) and it is called Side Scatter (SSC). 
It was pointed out by Kerker (1969) that as particle radius increases, the intensity of FSC is larger and increases faster than light scattered in other directions. Also, SSC is affected by the internal structure of a particle. It is confirmed that a particle with complicated structure would have high side scatter signals. In a word, the FSC factor is correlated with the particle's size and that SSC factor depends on the distribution of the particle structure.

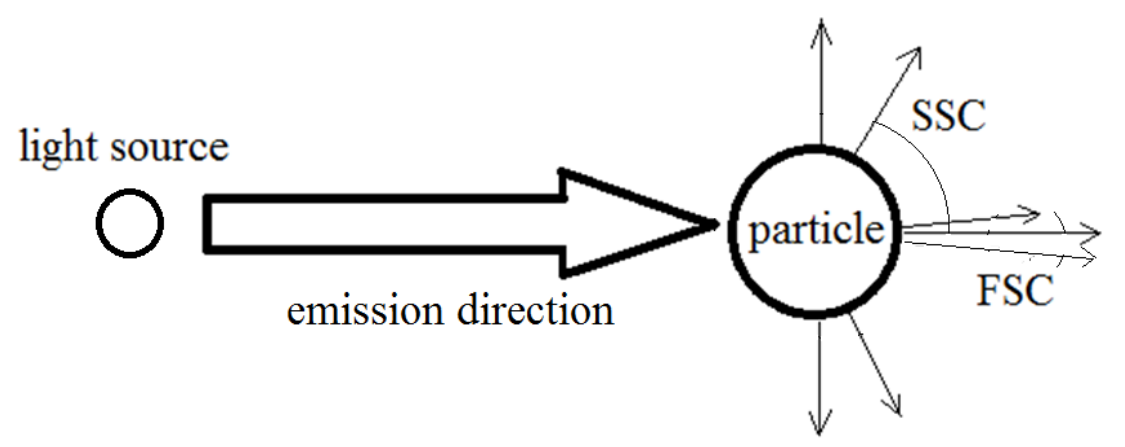

Figure I-2 Schematic representation light scattering (eventsHerzenberg et al. (2002)).

Light-blocking technique is another optical method also used in particle detection. When light strikes a particle, besides the reflected components, there is also one part of the light which is absorbed by the particle and one part which pass through the particle. The portion of each component is determined by the material light properties of the sample particle. Light-blocking technique considers the absorbed and reflected light away from the detector. The basic schematic is shown on Figure I-3. The photo-detector detects the sudden the light intensity variation from the detection region when a particle blocks the light. 
There are several papers which discussed the use of scattered light or light-blocking to obtain information about the size and shape of particles (Dubelaar et al. (1999), Cunningham and Buonnacorsi (1992), and Kerker (1983), Xiang et al. (2005)). It can be understood that scattered light method is more sensitive than light-blocking method because scattered light method detects the reflected light while light-blocking method detects the darkness. However, Howard (2005) mentioned that light scattering is a relatively complex phenomenon and the present mathematical correlation function which correlates FSC to particle size is not always correct.

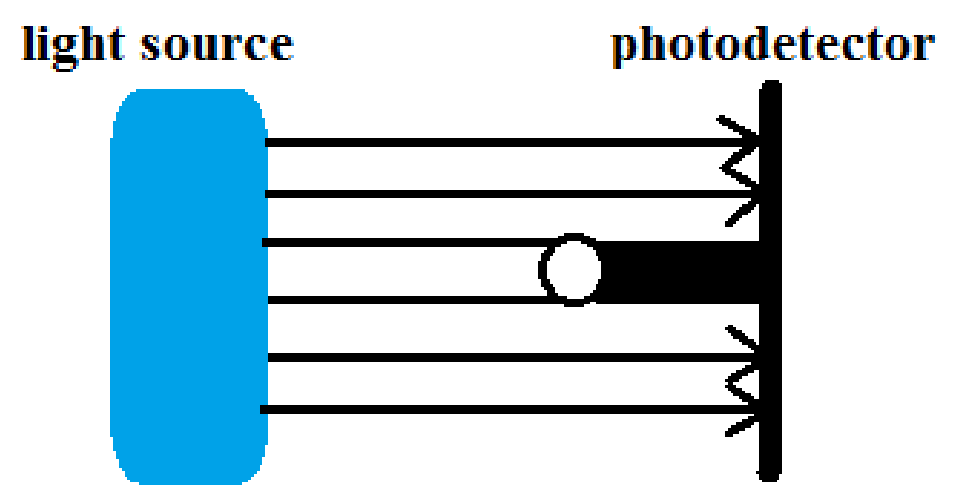

Figure I-3 Light-blocking technique (Zhang et al. (2009)).

Another physical mechanism that is used to detect the particles is called Coulter principle. In 1947 Wallace H. Coulter first discovered the so called Coulter principle when he conducted an electrical experiment. The traditional Coulter Counters include two containers of solution with particles and a single pore or a micro channel which is used to connect the containers. Coulter found that the electrical resistance variation can be directly connected to the existence of particles (which had lower electrical conductivities than the medium). He concluded that the electrical property variation could be used to 
determine the size and number of particles suspended in the electrolyte. This phenomenon is called the Coulter Counter Principle, and this kind of device is called Coulter Counter. Figure I-4 shows the schematic of the traditional Coulter Counter. It consists of two containers which are connected by an aperture. The left container has a higher fluid level than the right one. The difference in fluid level is used to keep the electrolyte moving from left to right. Several particles with low conductivities are suspended in conductive fluids. When particles move through the small aperture, the existence of the particles increases the total electrical resistance and therefore the current value drops.

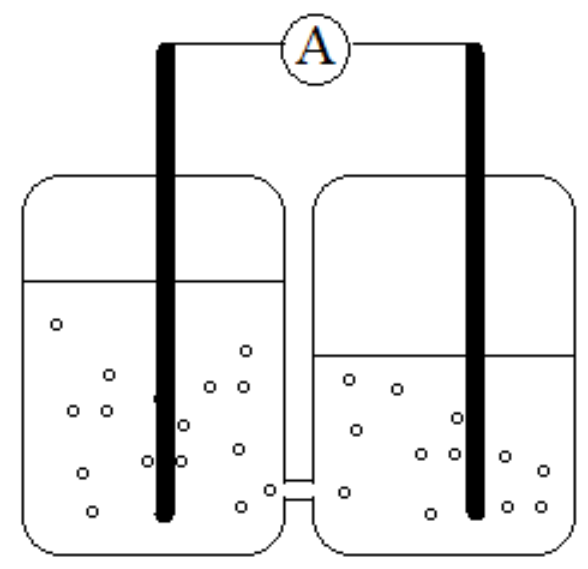

Figure I-4 Basic schematic of a traditional Coulter Counter.

The design of the aperture (or short micro channel) is the key idea of the traditional Coulter Counter because the conductivity of the detection region must be able to change obviously due to the movement of the particles. The current signal variation is tiny if the particles move just in the two containers. There are two reasons that cause the tiny variation. First, the particle size is really small compared with the size of the two 
containers; second, the electric field line distribution is coarse in the two containers except in the aperture region, so the small particles can only block few electric field lines. The electric resistance model of the Coulter Counter can be simplified to the model shown in Figure I-5. The red line on the left represents the high potential electrode. The blue line on the right represents the low potential electrode. The electric field lines distribution is shown in Figure I-5. Sun and Morgan (2010) pointed out that only particles with size close to that of the aperture diameter can be detected. We can see that a small particle in either of the containers would block a relatively small proportion of the electric field lines, so does not have a strong impact on current between the electrodes. However, when a particle with size close to that of the aperture is in the aperture, a large fraction of the electric field lines is blocked, causing a dramatic loss of current.
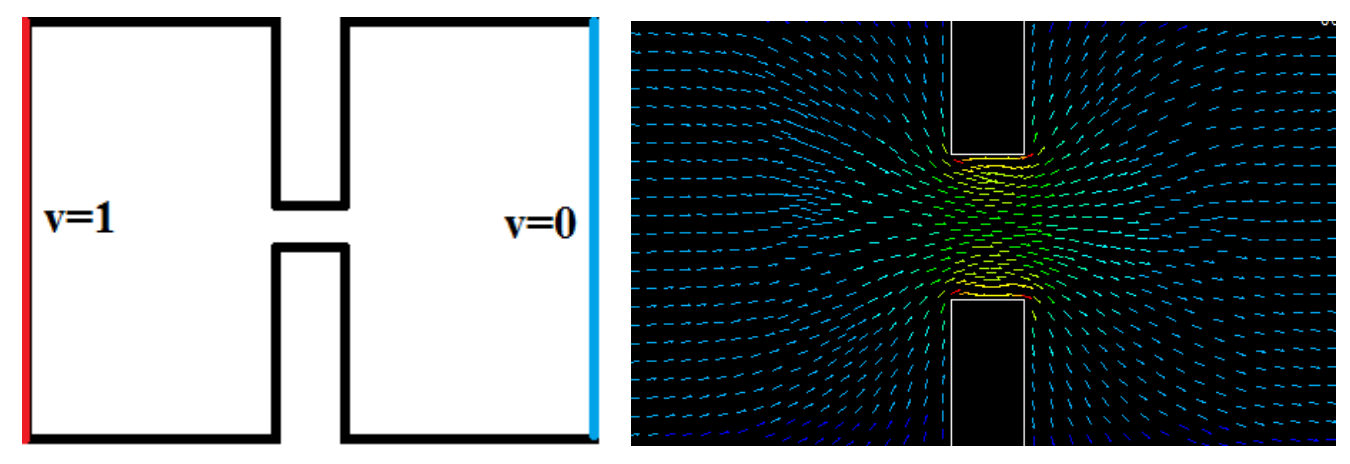

Figure I-5 The simplified electrical model for Coulter Counter.

Besides these three methods, there are also some other methods which are used to detect particles. For example, capacitance detection method (it measure the capacitance variation by apply the AC power. It can be used to qualify the polarization influence of DNA and detect the nucleus of some cells) is also widely used in detecting the cell 
structure. Sohn et al. (2000) reported its application in the field of DNA content of eukaryotic cells. Another method is called fluorescence method. The basic principle of this method is: some particles can launch a light when they are excited to higher electronic states. Some papers also called this method as Fluorescence-activated cell sorting (FACS). The device can recognize the particle information based on the quantitative fluorescent signals. Limited by the dissertation length, other detection methods are summarized on Table I-1.

\begin{tabular}{|c|c|c|}
\hline Method & Principle & Advantages/disadvantages \\
\hline Light scattering & $\begin{array}{l}\text { Detect the reflected light by the } \\
\text { particle. }\end{array}$ & $\begin{array}{l}\text { Mathematical correlation function } \\
\text { is not always correct. }\end{array}$ \\
\hline Light blocking & $\begin{array}{l}\text { Detect the darkness by the } \\
\text { particle. }\end{array}$ & $\begin{array}{l}\text { Less sensitive than light scattering } \\
\text { method but cheaper. }\end{array}$ \\
\hline Coulter Counter & $\begin{array}{l}\text { Detect the current variation } \\
\text { which is caused by the particle. }\end{array}$ & $\begin{array}{l}\text { Easy to fabricate, but only particle } \\
\text { size can be got. }\end{array}$ \\
\hline $\begin{array}{l}\text { Capacitance } \\
\text { detection }\end{array}$ & $\begin{array}{l}\text { Detect the capacitance variation } \\
\text { which is caused by the particle. }\end{array}$ & $\begin{array}{l}\text { Frequency modulation controller is } \\
\text { necessary. Be sensitive to probe } \\
\text { the polarization response. }\end{array}$ \\
\hline Fluorescence & $\begin{array}{l}\text { Detect the fluorescence which } \\
\text { is stimulated by photon } \\
\text { absorption. }\end{array}$ & $\begin{array}{l}\text { Particles must have fluorescent } \\
\text { characteristics. }\end{array}$ \\
\hline
\end{tabular}

Table I-1 Several particle detection methods. 


\subsection{Previous work of microfluidic Coulter Counter}

The advancement of micro technology has made it possible to design portable Coulter Counters which are smaller than the size of a fingertip. In this dissertation, there are two aspects that are worthy to be our research targets: (1) how to control the movement of the particles by pushing them into the detection region; (2) The electrical field configuration and the signal response based on the selected configuration. In the following parts the dissertation will illustrate the two aspects individually.

As we mentioned above, the particle trajectory is one research target. Several techniques are used to control the particle trajectory. Using an aperture (or orifice) is an available method to control the particle trajectory. Table I-2 shows several papers which studied this kind of design. One schematic of the aperture design by Ashish (2006) is shown in Figure I-6. The aperture was fabricated by two Polymer membranes. The two electrodes were inserted through two holes which were located on both sides of the micro

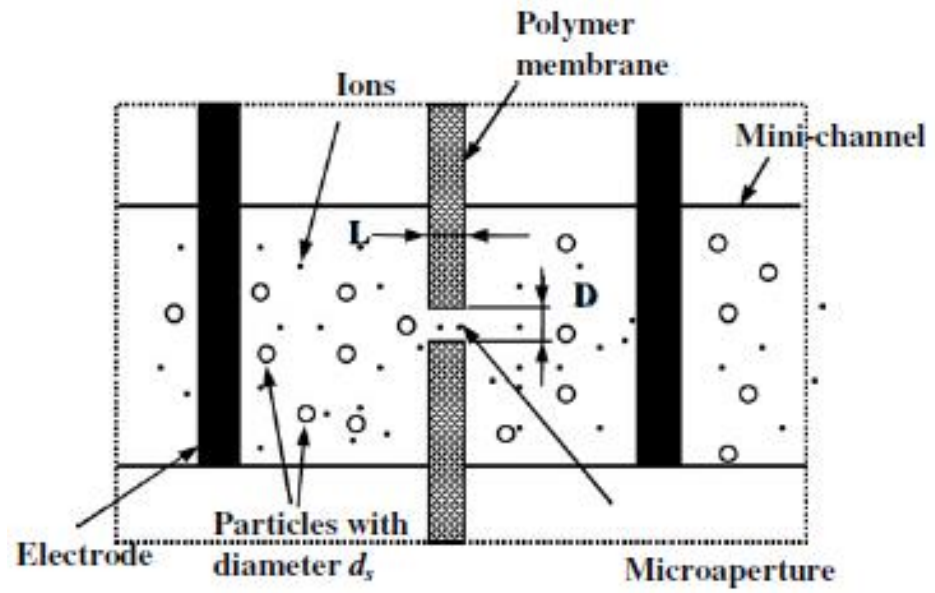

Figure I-6 The front view of a multi-aperture Coulter Counter (Ashish (2006)). 
aperture. The whole device had four parts which were similar to what Figure I-6 shows. This design can improve the particle detection range (the size of the four apertures can be different) and rate.

However, there are some disadvantages and risks for the aperture design. Scott et al. (2008b) illustrated a phenomenon that the particles maybe adhere to the walls of the aperture or even block the aperture because the size of the aperture is designed to get close to the size of the sample particles. It adds undesirable background noise in the electrical signals. What's more, it was mentioned by Howard (2005) that the particle could clog the channel. Even an 80-micron diameter orifice will be clogged several times a day by particles with 10-20 micron meters diameter. Also, the detection range of the particle size is limited since it is not easy to change the aperture size once the device is fabricated. Some designs integrate several apertures with different sizes into one microchip, this can improve the detection range a little bit but it cannot solve the problem fundamentally.

\begin{tabular}{|l|l|c|}
\hline $\begin{array}{c}\text { Research } \\
\text { method }\end{array}$ & \multicolumn{1}{|c|}{ Design/conclusion } & Refs \\
\hline Experiment & $\begin{array}{l}\text { An aperture was designed by a short micro channel } \\
\text { which was connected at the bottom of two large } \\
\text { reservoirs. }\end{array}$ & $\begin{array}{c}\text { Saleh and } \\
\text { Sohn } \\
(2003)\end{array}$ \\
\hline Experiment & $\begin{array}{l}\text { Two large micro channels were connected by a a } \\
\text { short micro channel. Particle moved in the short } \\
\text { micro channel to generate the resistive pulses }\end{array}$ & $\begin{array}{c}\text { Song et al. } \\
(2010)\end{array}$ \\
\hline Experiment & $\begin{array}{l}\text { A micro aperture was formed by two polymer } \\
\text { membranes. Two vertical electrodes were } \\
\text { surrounded by the solution. }\end{array}$ & $\begin{array}{c}\text { Ashish } \\
(2006)\end{array}$ \\
\hline Experiment & $\begin{array}{l}\text { A nano aperture was fabricated by introducing a } \\
\text { nanotubule. The detection size can get 2nm. }\end{array}$ & $\begin{array}{l}\text { Kobayashi } \\
\text { and Martin } \\
(1997)\end{array}$ \\
\hline Experiment & Test the signal response and confirmed that the & Cowan \\
\hline
\end{tabular}




\begin{tabular}{|l|l|c|}
\hline & $\begin{array}{l}\text { aperture can be used to detect the particles which } \\
\text { size are over a range of } 2 \% \text { to } 60 \% \text { of the aperture } \\
\text { diameter. }\end{array}$ & $\begin{array}{c}\text { and } \\
\text { Harfield } \\
(1990)\end{array}$ \\
\hline
\end{tabular}

\section{Table I-2 Research situation for aperture design.}

Another technique to control the particle trajectory is called hydrodynamic focusing method. The working principle of this technique can be explained by Figure I-7. It shows a Coulter Counter which uses two dimensional hydrodynamic focusing. In this design two sheath streams coming from the top and bottom inlets to focus the sample stream which comes from the middle inlet. Table I-3 shows several published papers which introduced the design for hydrodynamic focusing.

The hydrodynamic focusing technology has two sub-types. One type is called two dimensional hydrodynamic focusing. Figure I-7 shows its basic schematic. The sample stream under the impact of this kind of hydrodynamic focusing can only be adjusted in one direction. Several published papers have talked this issue (Pamme (2007), KersaudyKerhoas et al. (2008), Kulrattanarak et al. (2008), Tsutsui and Ho (2009)).

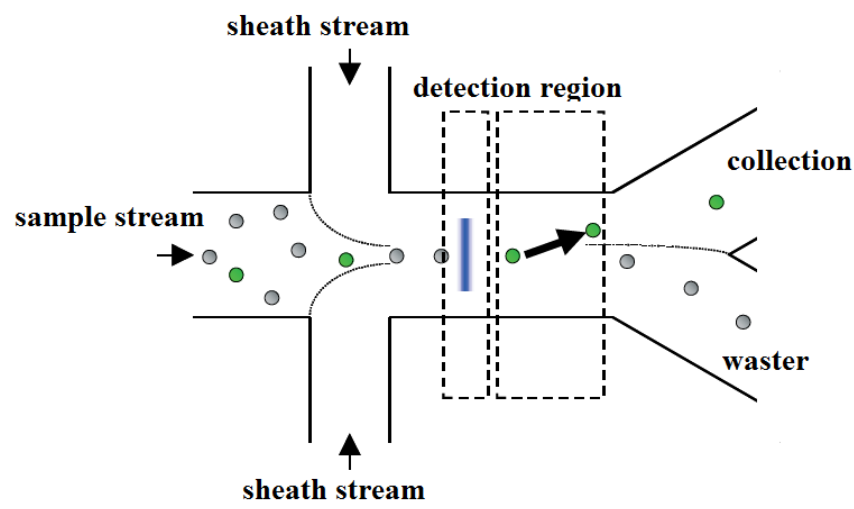

Figure I-7 Hydrodynamic focusing for detection (Wang et al. (2005)). 
However, there are also some disadvantages for the two dimensional hydrodynamic focusing. Considering the schematic as Figure I-8 shows, the two dimensional hydrodynamic focusing only focuses on one direction of the micro channel. This will lead some signal variation because the sample stream is not focused on the vertical direction. The particle can still move far away from the detection region by staying at different heights (Huh et al. (2005), Chung and Kim (2007), Ateya et al. (2008)). In order to overcome this drawback, another sub-type (called three dimensional hydrodynamic focusing) is created. The sample stream under the impact of the three dimensional hydrodynamic focusing can be adjusted in two directions. The difference of these two kinds of focusing methods is shown in Figure I-8. For the three dimensional hydrodynamic focusing, the size of the sample stream can be controlled by adjusting the sheath and sample stream flow rates from two directions. (close to the particle size). This will increase the signal sensitivity.

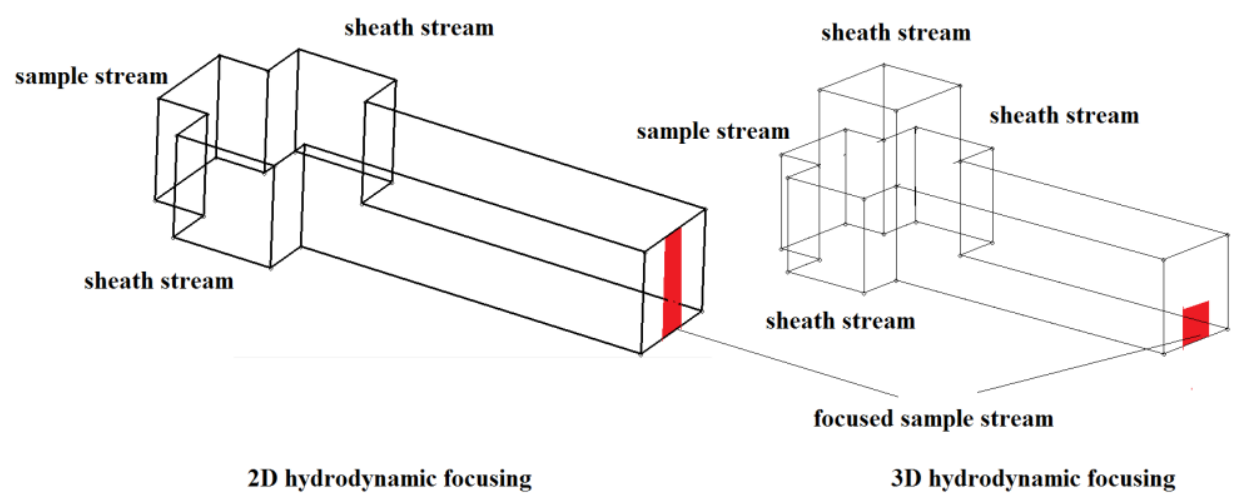

Figure I-8 Two types of hydrodynamic focusing.

Table I-3 lists several papers which focus on two-dimensional and three-dimensional hydrodynamic focusing. Scott et al. (2008a) designed a micro channel network which is 
shown in Figure I-9. Design (b) just used two sheath flows to focus on one direction. In order to get the three dimensional hydrodynamic focusing, design (c) decreased the height of the sample stream inlet so the sheath stream can flow around the sample stream. Design (d) used a stair-stepping geometry to focus the sample stream close to the bottom surface of the downstream channel.

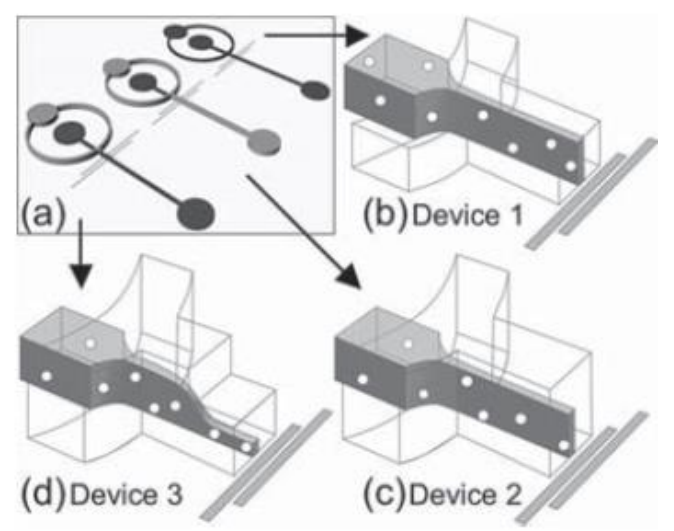

Figure I-9 Designs of 2D/3D hydrodynamic focusing (Scott et al. (2008a)).

Meanwhile there are also some novel designs which achieve the three dimensional hydrodynamic focusing by some unusual channel configurations. Howell et al. (2008) showed a three dimensional hydrodynamic focusing by using chevrons-based micro channel, as it is shown in Figure I-10. In this design, there were also two sheath flows which were used to focus the sample stream. Besides that, the top and bottom surfaces of the channel were cut by a pair of chevrons. These chevrons pushed the sheath flow from the side to the top and bottom regions of the channel; as a result, the sample stream was focused vertically. The similar design was found inTsai et al. (2008) and Sato et al. (2007). 


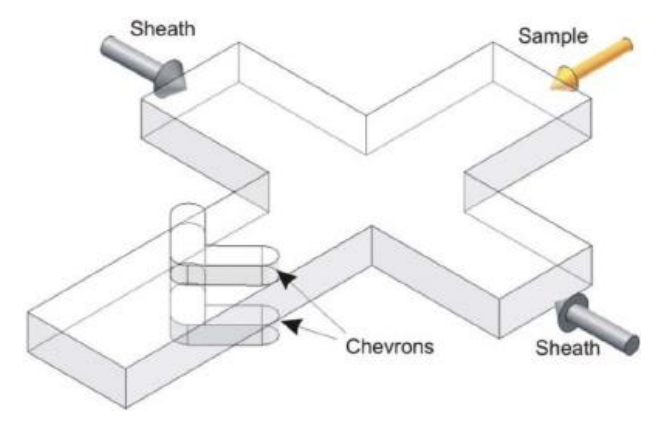

Figure I-10 Chevron-based sheath flow design. (Howell et al. (2008)).

Another special design was introduced recently by Lee et al. (2009). He reported a three dimensional hydrodynamic focusing with only one sheath stream. He extracted the idea from Dean-Flow phenomenon which was mentioned by Berger (1983) on his device. An array of contraction and expansion regions were designed in the micro channel in order to generate Dean-like Flow, as shown schematically in Figure I-11. Dean vortices were

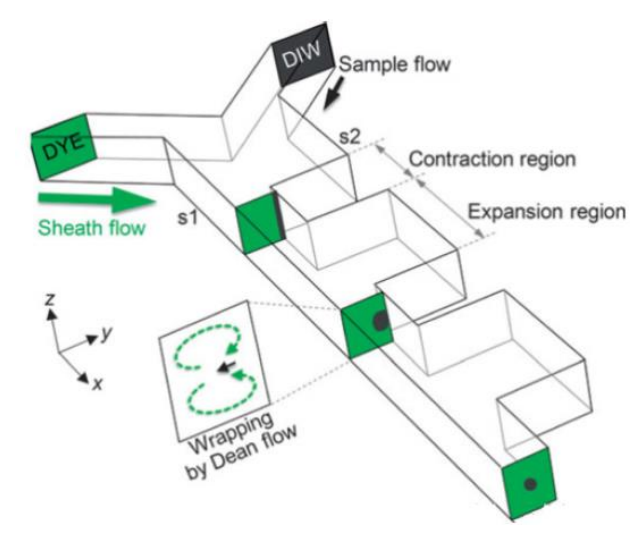

Figure I-11 3D hydrodynamic focusing with one sheath stream (Lee et al. (2009)). created at the contraction areas and it could influence the sheath and sample streams in the expansion regions. As a result, the sheath stream vertically focused the sample stream. Also, the Dean-Flow phenomenon can push the focused sample stream drifts to the outer 
corner of contraction area; this could focus the sample stream horizontally. The similar designs can be found in Mao et al. (2007).

\begin{tabular}{|c|l|c|}
\hline $\begin{array}{c}\text { Research } \\
\text { method }\end{array}$ & \multicolumn{1}{|c|}{ Design/conclusion } & Refs \\
\hline Experiment & $\begin{array}{l}\text { Design A: The sample stream was only focused by } \\
\text { the two sheath streams. Design B: The sample } \\
\text { stream came from a chimney which was set at the } \\
\text { bottom of the micro channel. The sample stream } \\
\text { was surrounded by the sheath stream. }\end{array}$ & $\begin{array}{c}\text { Wolff et } \\
\text { al. (2003) }\end{array}$ \\
\hline Experiment & $\begin{array}{l}\text { A sample stream was injected from the center } \\
\text { position (port II) of the micro channel and was } \\
\text { focused by multiple sheath streams (port I, III and } \\
\text { IV). A stepped channel was used to focus the } \\
\text { collected stream in order to push the stream close to } \\
\text { the detected region. }\end{array}$ & $\begin{array}{c}\text { Simonnet } \\
\text { and } \\
\text { Groisman } \\
(2006)\end{array}$ \\
\hline Experiment & $\begin{array}{l}\text { Three different designs were tested in order to } \\
\text { investigate the impacts of the two dimensional/three } \\
\text { dimensional hydrodynamic focusing and their } \\
\text { differences. }\end{array}$ & $\begin{array}{c}\text { Scott et al. } \\
(2008 \mathrm{a})\end{array}$ \\
\hline Experiment & $\begin{array}{l}\text { There were four sheath streams that were used to } \\
\text { focus the sample stream twice. This "double } \\
\text { focusing" technique can give a relative more } \\
\text { precisely control of the focused sample stream size. }\end{array}$ & $\begin{array}{c}\text { Kummrow } \\
\text { et al. } \\
(2009)\end{array}$ \\
\hline Experiment & $\begin{array}{l}\text { There were two sheath flows which were used to } \\
\text { focus the sample stream. Besides that, the top and } \\
\text { bottom surfaces of the channel were cut by a pair of } \\
\text { chevrons. This design can push the sheath flow } \\
\text { flowing from the side to the top and bottom region } \\
\text { of the channel, as a result, the sample stream was } \\
\text { focused vertically. }\end{array}$ & $\begin{array}{l}\text { Howell et } \\
\text { al. (2008) }\end{array}$ \\
\hline $\begin{array}{l}\text { An array of contraction and expansion regions were } \\
\text { designed in the micro channel in order to generate } \\
\text { the Dean-Flow. This can be treated as a horizontal } \\
\text { and a vertical focusing. }\end{array}$ & $\begin{array}{c}\text { Lee et al. } \\
\text { (2009) }\end{array}$ \\
\hline
\end{tabular}

Table I-3 Research situation for hydrodynamic focusing from experiment aspect.

Besides these results that mentioned above, several theorems and numerical simulations were reported during the last decades in this area. Table I-4 shows parts of them. Wu 
(2005) presented an analytical solution about the concentration distribution by assuming the velocity is uniform distribution along the whole fluid field. (Figure I-12). They also performed a numerical simulation for the concentration distribution at the certain cross section positions. In his dimensionless analytical solution, $x^{*}$ is the ratio of $x / W$ ( $W$ is the width of the $2 \mathrm{D}$ channel $), y^{*}$ is the ratio of $y / W$ and $c^{*}$ is the ratio of $c / C_{0}\left(C_{0}\right.$ is the concentration value of the sample stream in the middle). $P e$ is the Peclet number (characteristic length multiples velocity and divides the diffusion coefficient). ChihChang (2007) simulated the impacts of the main channel size to the focused sample stream width. The results confirmed that the sample stream can be focused successfully in the lower height/width aspect ratio of the main channel size. Hou et al. (2009) considered the three dimensional hydrodynamic focusing in a cytometer.

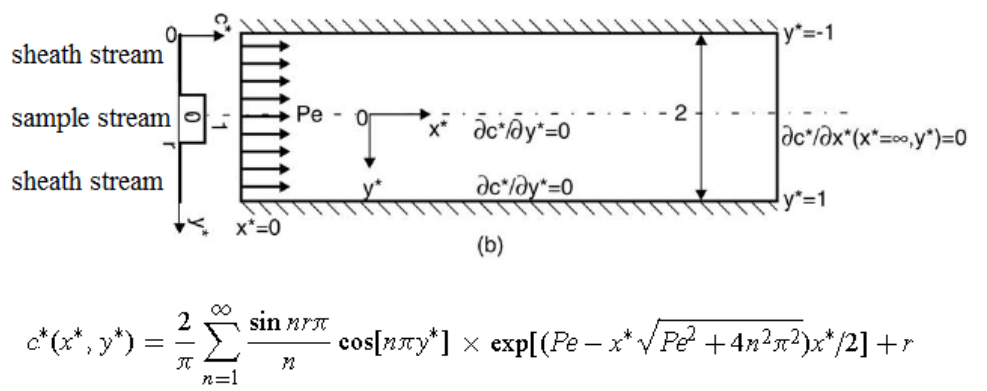

Figure I-12 The concentration distribution function (Wu (2005)).

The simulation gave a correlation about the relative focused width (focused sample stream width over main channel width) to the horizontal focusing ratio which is horizontal sheath stream flow rate over sample stream flow rate; the same thing was considered again about the correlation between relative focused height to the vertical focusing ratio. Both the two simulation results showed that when the focusing ratio 
increased, the relative focused width (or height) decreased. Lee (2001) proposed a three dimensional hydrodynamic focusing by just using one sheath inlet and gave an analytical formula about the focused sample stream width under the circular cross section situation. Yang and $\mathrm{Wu}(2006)$ showed the numerical simulation results about the focused sample stream width. A three dimensional grid was used and the solution showed that the simulated data agreed well with Lee (2001)'s work in a large range (velocity ratio about the sheath stream over the sample stream from 5 to 70). Ivorra et al. (2013) studied the ion concentration distribution under the impact of two dimensional hydrodynamic focusing. They found that the focusing effect was increased when the angle between the sheath flow and sample flow increased from zero to ninety degrees. Zhuang (2008) and Kunstmann-Olsen et al. (2012) used COMSOL to simulate a three dimensional hydrodynamic focusing case with three sheath streams and one sample stream. They showed the velocity contour profile of the cross section after the focused region and compared it with the experiment data. The agreement was good when the Reynolds number was no less than 0.5. Wu et al. (2012) and Guo et al. (2012) used COMSOL to simulate the impedance change in a microfluidic Coulter Counter. The simulation results showed that the resistance variation affected by the particle size and particle location. Lee (2004) showed a simulation for a two dimensional hydrodynamic focusing. The simulation work mainly focused on the variation of the flow rate ratio, sheath flow rate, sample flow width and the concentration distribution on the detection position, it confirmed that the hydrodynamic focusing technique can make the sample stream stay on a desired position and have a proper width at the detection position. Lee (2006) gave a 
mathematical expression for the ratio (the average focused stream velocity to the average stream velocity). They also studied the asymmetric hydrodynamic focusing effect

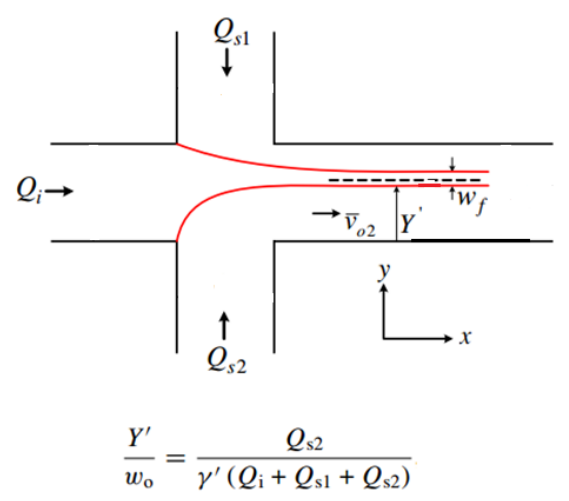

Figure I-13 The asymmetric hydrodynamic focusing (Lee (2006)).

and proposed a formula to calculate the ratio under the assumption of the case with two infinite parallel plates. In his analytical solution, $Y^{\prime}$ is the displacement from the bottom interface of the focused sample stream to the bottom channel boundary. $w_{0}$ is the width of the channel at the downstream. $Q_{s 1}$ and $Q_{s 2}$ is the fluid volume rate from the top and bottom inlets. $Q_{i}$ is the fluid volume rate from the center sample stream volume rate. $\gamma$ ' is the ratio between the mean rate from the bottom inlet to the total mean rate at the downstream from all the three inlets. Wang (2008) numerically studied the three dimensional hydrodynamic focusing technique by Fluent by considering the ion diffusion phenomenon. Their results showed clearly that the ion diffusion phenomenon happened at the interface between the sheath stream and the sample stream. In their study they also considered the factor of the rate ratio between the side stream and the sample stream. The results showed the concentration contour plot at different rate ratios. Lin et al. (2012) introduced a microfluidic device. It applied three dimensional hydrodynamic focusing by 
a single sheath stream. The sheath stream flowed from the vertical direction and it pushed the sample stream on the horizontal direction. Numerical simulations were processed to investigate the three dimensional hydrodynamic focusing performances. The results showed two different cases by changing the total flow volume rate and the ratio of the sheath /sample stream flow rates. It confirmed that this device can achieve the focusing goal by adjusting different flow volume rates and the ratio of the sheath/sample stream flow rates. Blonski et al. (2011) showed the three dimensional hydrodynamic focusing on a more fundamental level. A detailed study of the three-dimensional hydrodynamic focusing was performed by numerical simulation. They chose a three-inlets and oneoutlet micro channel with cross section of width $300 \mu m$ and height $400 \mu m$. The sample/sheath stream flow rates ratio and fluid viscosity were the two parameters they focused. The study showed the ratio between the sample stream rate and sheath stream rates $(1: 5,1: 10,1: 20,1: 40)$ and Reynolds number (Figure I-14 and Figure I-15, the color represents the percent of the focused sample stream). It showed when the Reynolds
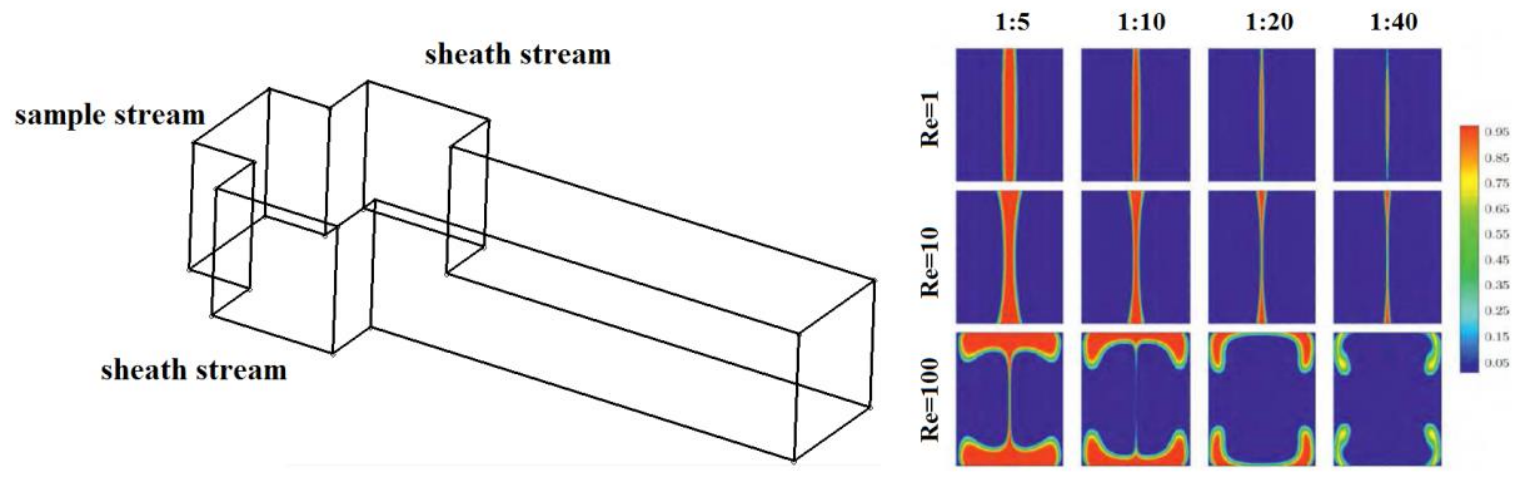

Figure I-14 Distribution of the focused sample stream for three different Reynolds number and four different focusing ratios (Blonski et al. (2011)). 
number was close to 1 . The focused sample stream width was near constant or has a convex shape. When the Reynolds number increased to 10 , the focused sample stream width had a concave shape and this phenomenon became more obvious as the Reynolds number increased. However, this conclusion did not consider the ion distribution phenomenon.

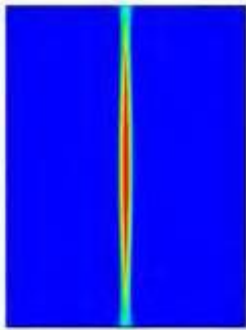

$\mathrm{Re}=0.1$

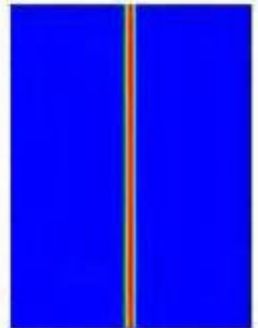

$\operatorname{Re}=5$

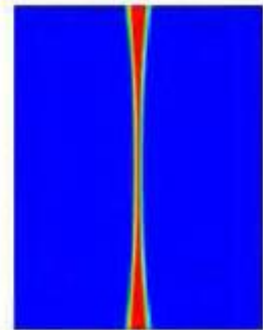

$\operatorname{Re}=10$

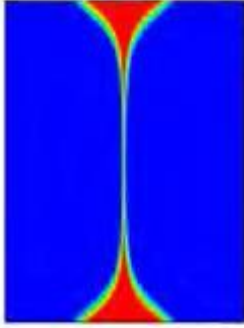

$\operatorname{Re}=25$

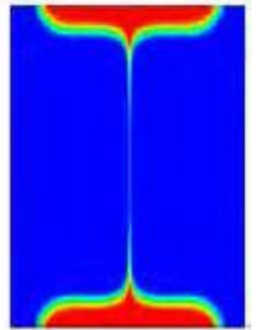

$\operatorname{Re}=50$

Figure I-15 Focused sample stream width considering the Reynolds number variation Blonski et al. (2011).

Ivorra et al. (2013) studied the ion concentration distribution under the impact of two dimensional hydrodynamic focusing. The paper studied the mixing time predicted both by two dimensional simulation and three dimensional simulation results. Jiang et al. (2013) conducted a three-dimensional simulation using COMSOL. Their study confirmed the impacts of the hydrodynamic focusing on the focused sample stream and the solvent concentration variation. Testa and Bernini (2012) simulated the cross-sectional fluorescence image by applying hydrodynamic focusing affect. Their results were in good agreement with experimental results. Wang et al. (2012) studied the performances of hydrodynamic focusing with two different fluids by considering the variation of the sheath/sample stream flow rate, the relative angle about sheath/sample stream direction and the micro channel height. 


\begin{tabular}{|c|l|c|}
\hline $\begin{array}{c}\text { Research } \\
\text { method }\end{array}$ & \multicolumn{1}{|c|}{ Design/conclusion } & Refs \\
\hline Analytical/ & $\begin{array}{l}\text { An analytical solution of the sample stream } \\
\text { concentration was introduced under the assumption } \\
\text { that the rate in the micro channel is uniform. They } \\
\text { also performed the numerical simulation for the } \\
\text { concentration distribution. }\end{array}$ & Wu (2005) \\
\hline Simulation & $\begin{array}{l}\text { A numerical simulation of the cross-sectional } \\
\text { profiles from the vertical focused stream was } \\
\text { shown. Also the impacts of the main channel cross } \\
\text { section geometry were simulated. }\end{array}$ & $\begin{array}{c}\text { Chih- } \\
\text { Chang } \\
\text { (2007) }\end{array}$ \\
\hline Simulation & $\begin{array}{l}\text { Simulated the focused sample stream width under } \\
\text { the impacts of the variation from the } \\
\text { vertical/horizontal stream flow rates. }\end{array}$ & $\begin{array}{c}\text { Hou et al. } \\
\text { (2009) }\end{array}$ \\
\hline Analytical & $\begin{array}{l}\text { Gave an analytical formula to calculate the focused } \\
\text { sample stream width based on the circle cross } \\
\text { section. }\end{array}$ & Lee (2001) \\
\hline Simulation & $\begin{array}{l}\text { An three dimensional simulation was processed and } \\
\text { the results agreed well with Lee (2001)'s work at a } \\
\text { large rate ratio range }\end{array}$ & Yang and \\
Wu (2006)
\end{tabular}




\begin{tabular}{|c|c|c|}
\hline & $\begin{array}{l}\text { Reynolds number. Also the asymmetric cases were } \\
\text { studied. }\end{array}$ & \\
\hline Analytical & $\begin{array}{l}\text { Gave an analytical solution about the offset } \\
\text { displacement of the focused sample stream and the } \\
\text { rates ratio. }\end{array}$ & $\begin{array}{l}\text { Domagals } \\
\text { ki (2011) }\end{array}$ \\
\hline Simulation & $\begin{array}{l}\text { Three dimensional simulation by considering the } \\
\text { diffusion phenomenon }\end{array}$ & $\begin{array}{l}\text { Narayan } \\
(2004)\end{array}$ \\
\hline Simulation & $\begin{array}{l}\text { It showed the focused sample stream profile by } \\
\text { three dimensional simulation. }\end{array}$ & $\begin{array}{l}\text { Hairer } \\
(2007)\end{array}$ \\
\hline Simulation & $\begin{array}{l}\text { Numerical simulation for the ion concentration } \\
\text { distribution under the design of chevron-shaped } \\
\text { grooves micro channel }\end{array}$ & $\begin{array}{l}\text { Hashemi } \\
\text { et al. } \\
(2010)\end{array}$ \\
\hline Simulation & $\begin{array}{l}\text { Numerical simulation of hydrodynamic focusing. It } \\
\text { showed the volume fraction of the sample stream }\end{array}$ & $\begin{array}{l}\text { Piotr } \\
(2007)\end{array}$ \\
\hline
\end{tabular}

Table I-4 Research for hydrodynamic focusing from analytical/numerical aspects.

As the literature review mentioned above, many papers studied on the focused sample stream width. Both analytical and simulation results were presented. However, only few papers paid attention to the existence of diffusion phenomenon. Also the simulation of the particle motion is time consuming and this topic was talked rarely in the published papers. This dissertation will simulate the hydrodynamic focusing technique by considering the impacts of these topics.

Besides the hydrodynamic focusing technique applied on the particle trajectory, another interested topic which we focus in this dissertation is the electrical field configuration for micro Coulter Counter including the signal response based on the selected configuration. Table I-5 introduced several published papers about this topic. Coplanar electrodes distribution is a common way to set up the electrical field configuration. Nieuwenhuis et al. (2004) tested a microfluidic Coulter Counter by using three sheath streams which surrounded a sample stream (Figure I-16). The focused sample stream width was 
dynamically adapted to the size of the tested particle. Two metal electrodes were fixed on the bottom surface of the micro channel. He studied the impact of the focused sample stream dimension to the electrical signal variation and confirmed that the current signal variation can be adjusted to a high level by changing the relative sheath/sample stream flow rates and making the focused sample stream size close to the particle.

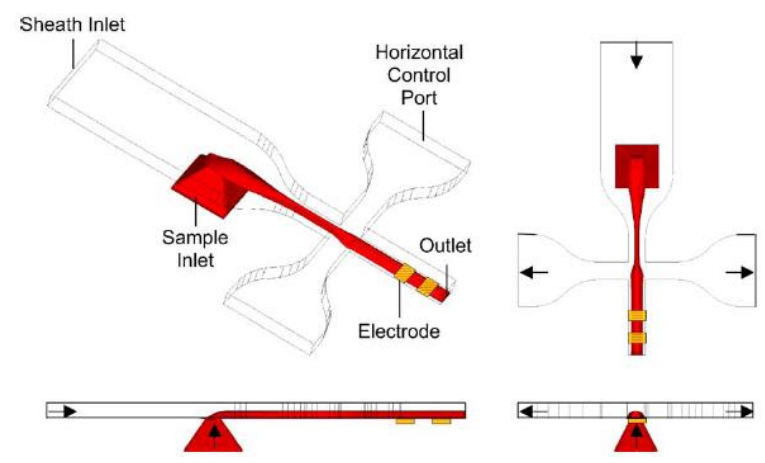

Figure I-16 The sample stream is focused by three sheath flows (Nieuwenhuis et al., 2004).

Rodriguez-Trujillo et al. (2007) designed a micro Coulter Counter which is shown in Figure I-17. The conductive sample stream flowed into the device from the top of the

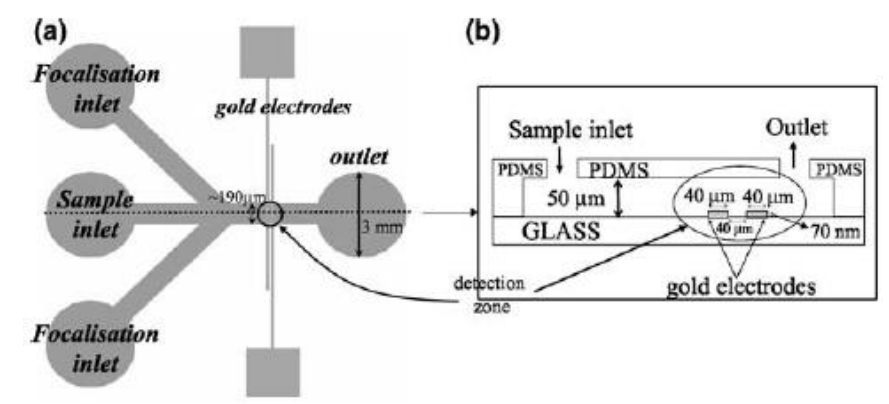

Figure I-17 The schematic for electrical detection method configuration under the hydrodynamic focusing (Rodriguez-Trujillo et al., 2007). 
channel. It was focused by two sheath flows with the flow direction angle about 45 degrees. The electrical signal at the detection region was monitored and changed when particles flowed into the detection region. This experiment compared the electrical signal variation under the 2D/3D hydrodynamic focusing. The similar work were done again in Rodriguez-Trujillo et al. (2008).

\begin{tabular}{|c|l|l|}
\hline $\begin{array}{c}\text { Research } \\
\text { method }\end{array}$ & \multicolumn{1}{|c|}{ Focused area } & \multicolumn{1}{|c|}{ Refs } \\
\hline Experiment & $\begin{array}{l}\text { It studied the correlation of the measured } \\
\text { resistance amplitude and the off-axis } \\
\text { particle position. }\end{array}$ & $\begin{array}{l}\text { Berge et al. } \\
(1990)\end{array}$ \\
\hline Experiment & $\begin{array}{l}\text { It Did the experiment to compare the } \\
\text { electrical signal variation under the } \\
\text { 2D/3D hydrodynamic focusing. }\end{array}$ & $\begin{array}{l}\text { Rodriguez et } \\
\text { al. (2008) } \\
\text { Rodriguez } \\
\text { (2007) }\end{array}$ \\
\hline Experiment & $\begin{array}{l}\text { Experimental studied the impact of the } \\
\text { aperture dimension to the resistance } \\
\text { signal variation. }\end{array}$ & $\begin{array}{l}\text { Nieuwenhuis } \\
\text { et al. (2004) }\end{array}$ \\
\hline Experiment & $\begin{array}{l}\text { It designed a micro Coulter Counter } \\
\text { which can use four detection channels } \\
\text { simultaneously. }\end{array}$ & Zhe (2007) \\
\hline Experiment & $\begin{array}{l}\text { Measured the particle size and the } \\
\text { particle concentration by DC power } \\
\text { supply. }\end{array}$ & Lee (2006) \\
\hline Experiment & $\begin{array}{l}\text { It designed a micro-fabricated aperture } \\
\text { Coulter Counter and tested two different } \\
\text { sizes of apertures. }\end{array}$ & $\begin{array}{l}\text { Roberts et } \\
\text { al. (1999) }\end{array}$ \\
\hline Experiment & $\begin{array}{l}\text { Experimental tested the impact of the } \\
\text { electrodes length and gap for micro } \\
\text { Coulter Counter. }\end{array}$ & $\begin{array}{l}\text { MADHURI } \\
\text { (2007) }\end{array}$ \\
\hline Experiment & $\begin{array}{l}\text { Experimental fabricated a micro Coulter } \\
\text { Counter and tested the idea that the } \\
\text { capacitance variation can be used to } \\
\text { detect the particle size and type. }\end{array}$ & $\begin{array}{l}\text { Kawaguchi } \\
\text { et al. (2012) }\end{array}$ \\
\hline
\end{tabular}

Table I-5 Published papers about the electrical field configuration and signal variation. 
Face-to-face electrodes distribution is another electrodes configuration to detect the particle. Bernabini et al. (2011) did a microfluidic Coulter Counter by introducing oil as the sheath flow in order to increase the signal sensitivity. One pair of opposing electrodes was fixed on the top and bottom surface of the micro channel boundary. The system can detect the micro particle as small as one micro meter diameter by changing the relative rates of the sample/sheath streams. The experiment confirmed that the focusing technique increases the sensitivity of the system.
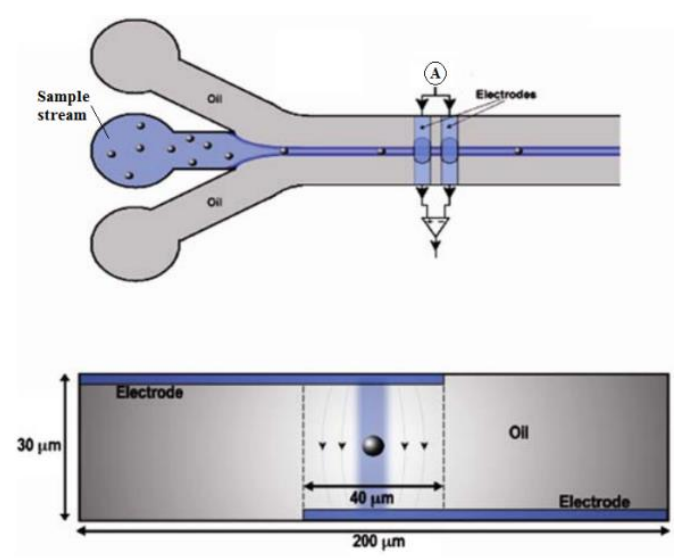

Figure I-18 A microfluidic Coulter Counter by choosing oil as the sheath stream (Bernabini et al., 2011).

Meanwhile, some scientists focused on the relative theorems and numerical simulations to study the signal variation based on selected electrical field configuration. Several analytical solutions for the resistance variation are listed in Table I-6. Maxwell and Thomson (1904) and Rayleigh (1892) gave an analytical solution to predict the resistance variation by assuming that the particle was non-conductive and the domain was infinite. The drawback of the solution by Maxwell is that his solution assumed that the particle radius was very small (approach to zero). DeBlois (1970) gave a new analytical solution, 
it was also available if the diameter ratio of the particle/channel was less than 0.5. Gregg and Steidley (1965) had another solution and it was available for the particles with the diameter of $0.5 \sim 0.8$ of the channel diameter. Saleh (2003) revised the function which was used by DeBlois's equation. Recently, Chapman and Sohn (2011) studied the resistance variation when a spherical particle passes through a cylinder and gave an empirical formula to calculate the resistance variation. Also they concluded that if the resistance was very small compared to the total resistance, then the relative change in the current were equal in the magnitude to the relative change in the resistance. Qin (2009) and Qin et al. (2011) simulated the electrical resistance variation when non-conductive particles pass through a micro-channel. They investigated the impact of particle position, shape and orientation on the resistance variation and they showed that all these factors could affect the resistance variation dramatically. The particle size in these two papers was relatively large $(d / D>0.3$, where $d$ is the particle diameter and $D$ is the diameter of the micro channel). Gawad et al. (2001), Spencer and Morgan (2011) designed Coulter Counters by introducing a reference signal. In Gawad's design, one pair of electrodes was used to detect the current variation due to the movement of the particles while another pair of electrodes was used as the reference. They numerically studied the impedance variation when the particle passed through the four electrodes, and the signal curve showed one peak and one valley. Spencer's design was similar to Gawad's, but the former used four electrodes instead of two electrodes. They numerically studied the impedance sensitivity in three different electrode configurations and showed that the face-to-face electrodes configurations had better current sensitivity than coplanar electrodes configurations under their device parameters 


\begin{tabular}{|c|c|c|}
\hline $\begin{array}{l}\text { Research } \\
\text { method }\end{array}$ & Focused area & Refs \\
\hline Analytical & $\begin{array}{l}\text { Derived an analytical formula to predict the } \\
\text { electric resistance variation. }\end{array}$ & $\begin{array}{c}\text { Rodriguez } \\
\quad \text { et al. } \\
\quad(2008) \\
\text { Rodriguez } \\
\quad(2007)\end{array}$ \\
\hline Analytical & $\begin{array}{l}\text { Gave an analytical solution to predict the } \\
\text { resistance variation by assuming that the } \\
\text { particle was non-conductive and the } \\
\text { domain was infinite. }\end{array}$ & $\begin{array}{l}\text { Maxwell } \\
\text { and } \\
\text { Thomson } \\
\text { (1904) } \\
\text { Rayleigh } \\
\text { (1892) }\end{array}$ \\
\hline Analytical & $\begin{array}{l}\text { Investigated the small resistance variation } \\
\text { in Coulter Counter and summarized a } \\
\text { formula to depict the correlation between } \\
\text { the resistance and current. }\end{array}$ & $\begin{array}{l}\text { Chapman } \\
\text { and Sohn } \\
(2011)\end{array}$ \\
\hline Analytical & $\begin{array}{l}\text { Developed a new analytical solution, which } \\
\text { was available if the diameter ratio of the } \\
\text { particle/channel was less than } 0.5 \text {. }\end{array}$ & $\begin{array}{l}\text { DeBlois } \\
(1970)\end{array}$ \\
\hline Analytical & $\begin{array}{l}\text { Revised the solution from DeBlois (1970) } \\
\text { and gave a revised function. }\end{array}$ & $\begin{array}{l}\text { Saleh } \\
(2003)\end{array}$ \\
\hline Analytical & $\begin{array}{l}\text { Developed a new analytical solution, which } \\
\text { was available if the diameter ratio of the } \\
\text { particle/channel was from } 0.5 \text { to } 0.8 \text {. }\end{array}$ & $\begin{array}{l}\text { Gregg } \\
\text { and } \\
\text { Steidley } \\
(1965) \\
\end{array}$ \\
\hline Simulation & $\begin{array}{l}\text { Simulated the electrical resistance variation } \\
\text { under the impacts of particle position and } \\
\text { particle shape. }\end{array}$ & $\begin{array}{c}\text { Qin et al. } \\
\text { (2011) } \\
\text { Qin } \\
(2009)\end{array}$ \\
\hline Simulation & $\begin{array}{l}\text { Designed a reference electrodes system and } \\
\text { numerically studied the impedance } \\
\text { variation when the particle passed through } \\
\text { the detection region. }\end{array}$ & $\begin{array}{l}\text { Gawad et } \\
\text { al. }(2001)\end{array}$ \\
\hline
\end{tabular}




\begin{tabular}{|l|l|c|}
\hline Simulation & $\begin{array}{l}\text { Numerical simulated the resistance } \\
\text { variation under three different electrodes } \\
\text { configuration. }\end{array}$ & $\begin{array}{c}\text { Spencer } \\
\text { and } \\
\text { Morgan } \\
(2011)\end{array}$ \\
\hline Simulation & $\begin{array}{l}\text { Numerical simulated the impedance } \\
\text { variation by considering the pore size. }\end{array}$ & $\begin{array}{c}\text { Cho } \\
(2009)\end{array}$ \\
\hline Simulation & $\begin{array}{l}\text { Simulated the current variation at the full } \\
\text { time scale. Demonstrated that its algorithm } \\
\text { correctly described the experimentally } \\
\text { observed signals. }\end{array}$ & $\begin{array}{l}\text { Stober et } \\
\text { al. (2009) }\end{array}$ \\
\hline
\end{tabular}

\section{Table I-6 Published papers about the signal variation from analytical/simulation aspects.}

setting. Cho (2009) investigated the impedance variation with the presence of multiple micro-pores. It showed that the normalized resistance variation was linearly dependent on the pore radius, but decreased as the gap between the pores increased. Uehara et al. (2013) addressed theoretical methods to analyze the current variation of $\lambda$ DNA which passed through a nano channel. The analytical solution for the current when there was no particle in the detection was introduced to compare with the solution when particle passed through the nano gap. The result from their model was in agreement with their experimental data.

As the literature review mentioned above, the signal variation depends on many design parameters. Some papers studied analytical solutions that assumed the particle moves in an infinite medium. Most research papers assumed that the conductivity distribution is uniform. However, in my case, ion diffusion is important because of the concentration difference between the low conductivity sheath stream and high conductivity sample stream. The conductivity distribution is not uniform if we apply the hydrodynamic focusing technique. Meanwhile, few papers mentioned the impacts of the variation from 
electrodes position, size, sample/sheath rates, etc. This dissertation will simulate the signal variation by considering these factors.

\subsection{Challenges in the numerical study of Coulter Counter}

There are several difficult points that need to be considered before we start numerical simulation. They include the computing time under the situation of high grid density, simulation stability under the low Reynolds number, moving grid problem and electrical conductivity difference. The following part will introduce them individually.

\subsubsection{High grid density and small time interval requirements}

The numerical simulation for the convection-diffusion phenomenon that exists in the interface of the sheath stream and sample stream is a difficult problem to deal with. Based on the discussion above, the ion concentration of the sample stream is much higher than the ion concentration of the sheath stream. So the concentration gradient is relative high on the adjacent parts of the two different streams. In order to capture the concentration distribution correctly, sufficient grid density need be applied to the simulation domain. This will dramatically increase the computation time. Also, this case becomes even worse by considering that it happens under the low Reynolds number region. Since the concentration field simulation is processed based on the fluid field simulation, both of the two simulation cases share the same grid and same setting parameters such as time interval. Here the fluid is under the laminar situation and the Reynolds number $(1<\operatorname{Re}<10)$ is much smaller than our normal scale. If an explicit 
method is chosen to solve the problem, the time step $d t$ should follow the condition below. Here $\rho$ is the fluid density, $d x$ is the grid size and $\mu$ is the viscosity.

$$
d t<\rho \frac{(d x)^{2}}{\mu}
$$

This stability condition (Li and Renardy (2000)) is much more restrictive than the CFL (Courant-Friedrichs-Levy) number condition. The situation could be worse if we run two dimensional or three dimensional simulation. Since we mention that the requirements of the concentration simulation needs very small grid space interval, this will give a more restrictive condition for time interval based on the low Reynolds number condition. If we choose implicit method, we can choose a relative larger time interval, but the computing time is still tedious for each time iteration period because of the large matrix size that we need to solve during each time iteration. In this study, the grid space and time interval are considered carefully in order to get the correct solution under the acceptable time constraints.

\subsubsection{Moving mesh problem}

The particle motion in the micro channel is another topic to research. In order to capture the fluid field and ion concentration field correctly, the particle has a finite area or volume under the simulation process. This is different compared with many published papers which studied the particle motion in micro channels by just choosing a point to stand for the particle. However, In order to capture the particle motion, a problem (called moving grid) needs grid regeneration after each time interval has to be faced. Grid 
regeneration usually takes huge time to fulfill. In this study, a numerical solver called Overture will be used to solve the moving grid problem. The grid regeneration time is decreased under this algorithm which is chosen by Overture. Only the region which is close to the moving grid needs to be regenerated. Even that, the regeneration consuming time still needs be treated seriously. Also, the grid mesh should be carefully cooperated to avoid failure of grid regeneration.

\subsubsection{Ill-conditioned problem}

Grégoire (2007) pointed out that, for a given equation $A x=b$, if the condition number (defined as the ratio of the maximal and minimal singular values of $\mathrm{A}$ ) for the matrix $A$ is too large, we cannot get good solution from the numerical simulation. This is called an ill-conditioned problem. As we mentioned above, the particle conductivity can be much smaller comparing with the sample stream conductivity. This will cause an "illconditional" matrix problem. We will demonstrate the ill-condition problem with the following testing case. Assume that we have a domain, its size is one by one, we are going to simulate the electric potential distribution in this domain. For the grid point distribution, there are 25 points in this domain (as the Figure I-19 shows). We use $c(i, j)(1 \leq i \leq 5,1 \leq j \leq 5)$ to represent the value of dimensionless conductivity in the point $(i, j)$. The values of the conductivities for all 25 points are all one except these five points $(2,3),(4,3),(3,2),(3,4)$ and $(3,3)$. We make the simulation under three different scenarios: the values of the conductivities for the five test points are $1,0.5$ and 0.1 . The potential value for all the boundary sides is 0.5 except the bottom boundary is 1 . All the three cases are simulated and Figure I-20 shows the potential distribution contour of these 
three different cases. We can see that plot (a) reflects the reasonable physical phenomenon: the potential value is higher on the region which is close to the bottom boundary while it is lower on the region which is further from the bottom boundary. Also

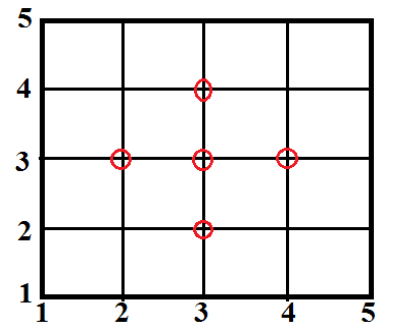

Figure I-19 The schematic of the test points by marking with red circles.

the total potential distribution is symmetric based on the symmetric boundary conditions and conductivity distribution. The plot (b) is the case that when the five tested points have lower conductivity 0.5 , in this situation, we can see that plot (b) does not match the symmetric distribution characteristic comparing with the potential distribution from plot

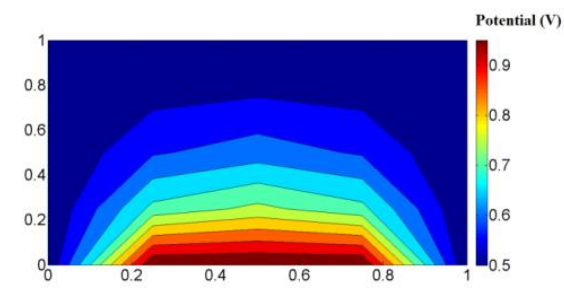

(a)

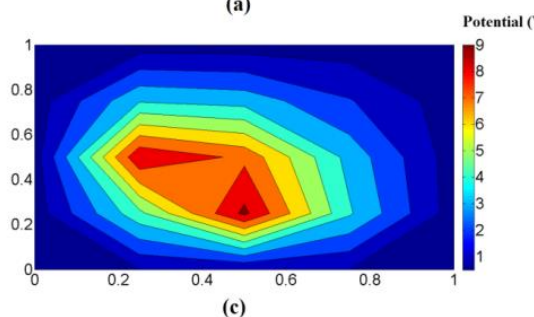

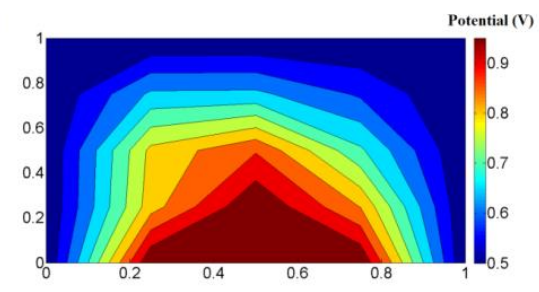

(b)

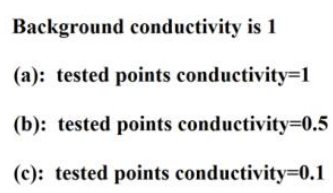

Background conductivity is 1

(c): tested points conductivity $=0.1$

Figure I-20 Simulation potential fields with three different conductivities of the tested points. 
(a). At last the plot (c) is totally wrong because the highest potential region should be the bottom boudary. This simulation test confirms that the "ill-conditional" matrix problem can make the simulation run abnormally and make the results totally meaningless. In order to conquer this problem, a new numerical difference method is used for our electrical potential simulation.

\subsection{Scope and objectives of this thesis}

This dissertation desires to use dimensionless numerical simulation to study the characteristics of hydrodynamic focusing numerically and include a relatively detailed investigation about the current signal variation under the impacts of the hydrodynamic focusing. In order the dimensionless study the fluid field and concentration field, three dimensionless numbers are considered in this project. They are listed below.

$$
\begin{gathered}
\operatorname{Re}=\frac{\rho L V}{\mu} \\
P e=\frac{L V}{D} \\
S c=\frac{\mu}{\rho D}
\end{gathered}
$$

Here $R e$ is the Reynolds number $P e$ is the Peclet number and $S c$ is the Schmidt number ( $\rho$ is the fluid density, $L$ is the characteristic length, $V$ is the characteristic velocity, $\mu$ is the dynamic viscosity of the fluid and $D$ is the diffusion coefficient). Our Reynolds number range is from one to ten, the fluid flow is considered as steady, incompressible 
flow and the particle is chosen as small circle/sphere (the diameter ratio of sphere/channel width is no more than $8 \%$ ). The Peclet number and Schmidt number are studied in the range from 1000 to 4000 ( $P e=830$ in Hinsmann et al. (2001), $P e=1500$ in Biddiss et al. (2004) and $S c=2400$ in Kamholz et al. (1999)). The ratio of conductivity between the particle and the sample stream is 0.1 . Same conductivity ratio is set for the sheath stream/ sample stream. Correlation between the ion concentration distribution and the hydrodynamic focusing configuration (diffusion coefficient, relative sheath/sample stream flow rates) will be discussed. Also the particle motion is considered in our research, the movement of the particles suspending in the sample stream will be simulated and the impacts of the hydrodynamic focusing to the single/multiple particles motion are researched. Besides that, the impacts of the hydrodynamic focusing to the current signal variation are analyzed including the variation of particle position, particle size, electrodes configuration, ion concentration and distribution deviation. Both two dimensional and three dimensional simulations are processed in order to understand these problems clearly. 


\section{Summary}

This chapter firstly introduces several particle detection methods. Then Coulter counter principle is studied and two important areas in this field are analyzed. For the particle trajectory, one important technique named hydrodynamic focusing is introduced. Its subtypes, typical design ideas, relative theorems and numerical simulation are introduced in the literature review. The review shows that few papers mentioned the ion diffusion phenomenon in this technique. Also the study of particle motion from the numerical simulation aspect is rarely seen from the published papers. Besides that, the current signal variation under the certain electrodes configuration is discussed. Some analytical solutions and numerical simulations used to predict the electrical signal variation are introduced. At last this chapter points three difficult problems that need to be solved in this dissertation. The large concentration difference between the sheath/sample stream and large fluid viscosity make the numerical simulation time consuming. The particle motion simulation needs to adjust the grid after each time step. This not only takes time but also has the risk of grid regeneration failure. The large conductivity difference between the particle and the sample stream could cause the "ill-conditioned" problem that may lead to a completely wrong result. In order to solve these problems, different grid density and time step are tested to find an appropriate grid distribution. A CFD package named "Overture" is used to solve the moving grid problem. A new center difference method is used to simulate the Laplace equation to avoid the "ill-conditioned" problem. 


\section{EQUATIONS AND NUMERICAL METHODS}

In order to simulate the signal variation in micro Coulter counter, the fluid, concentration and electrical equations need be solved serially. Here the fluid equation we choose to solve is Navier-Stokes equations; the concentration equation we choose to solve is convection-diffusion equation and the electrical equation we choose to solve is Laplace equation. Also here the particle motion phenomenon is considered to deal with. This chapter first introduces the governing equations we choose to solve, and then the numerical methods we choose to deal with these equations are discussed. 


\subsection{Governing equations}

For microfluidics, the Reynolds number is ranged between $10^{-6}$ and 10 (Todd (2005)). In our simulation, we mainly focus on cases with Reynolds numbers from 1 to 10 .

The laminar, incompressible flow behavior can be described by the Navier-Stokes equations and the continuity equation as below

$$
\begin{gathered}
\nabla \cdot \vec{u}=0 \\
\frac{\partial \vec{u}}{\partial t}+\vec{u} \bullet \nabla \vec{u}=-\frac{\nabla p}{\rho}+v \nabla^{2} \vec{u}
\end{gathered}
$$

where $v$ is the kinematic viscosity, $p$ is the pressure, $u$ is the velocity, $t$ is the time and $\rho$ is the fluid density.

The concentration distribution of the ion in the sample stream can be described by the convection- diffusion equation

$$
\frac{\partial c}{\partial t}+\vec{u} \bullet \nabla c=k \nabla^{2} c
$$

where $c$ is the concentration and $k$ is the molecular diffusivity coefficient.

By solving the Eq.(II.3), we get the ion concentration distribution. Since the conductivity is linearly dependent on ion concentration, here we can take the value of the ion concentration distribution as the electrical conductivity distribution on our simulation 
domain (Figure II-1). The next step is finding the electric potential distribution on the simulation domain based on the electrical conductivity distribution.

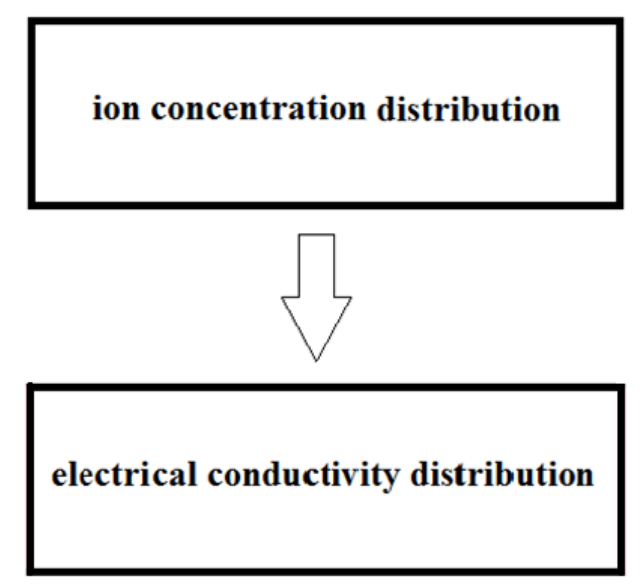

Figure II-1 The correlation between the electrical conductivity and the ion concentration.

The electrical potential distribution in a finite region can be described by the Laplace equation

$$
\nabla \bullet(\sigma \nabla \phi)=0
$$

where $\phi$ represents the potential and $\sigma$ is the electrical conductivity.

The current density and the value of the current through certain a cross section can be calculated by

$$
J=-\sigma \nabla \phi
$$




$$
I=\int_{y=a}^{y=b} J d y
$$

Where $a$ and $b$ are the vertical coordinate value of the two endpoints of the cross section as Figure II-2 shows.

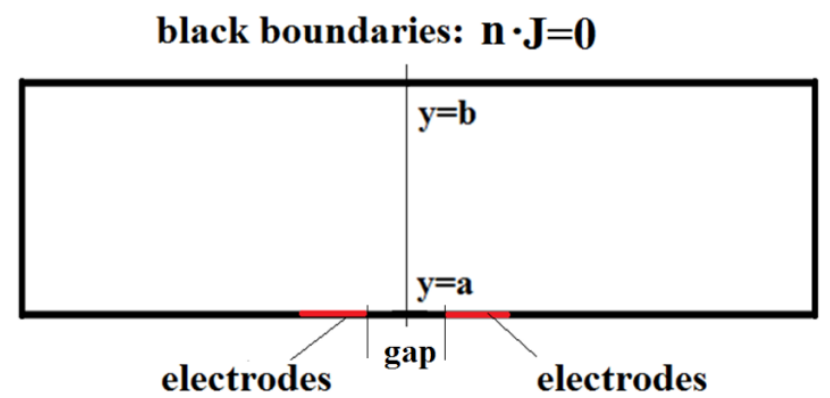

Figure II-2 The current integration through certain cross section.

\subsection{Numerical methods}

The governing equations for fluids [Eq.(II.1)\& Eq.(II.2)] are solved by using a PressurePoisson method on an overlapping grid. The method was introduced by Brown (1997). Both the convection and diffusion terms are discretized with a second-order accurate center difference scheme. The integration of time can be accomplished through a secondorder accurate Adams-Bashforth-Moulton predictor-corrector method which was introduced by Henshaw (1994). Since the flow field is not affected by the mass transfer, the mass convection-diffusion equation is decoupled from the Navier-Stokes equations. Eq. (II.3) is solved separately after the flow field is obtained.

The governing equations are discretized on overlapping grids. The overlapping grid method uses a set of component grids to cover the computational domain. As Figure II-3 
shows, the two components grids circle1 and circle 2 overlap in the grid background. Typically boundary-conforming structured grids are used to achieve high-quality representations of boundaries. It employs a Cartesian grid as the background grids so that the efficiencies inherent with such grids can be exploited. The irregular boundary associated with standard Cartesian grid methods takes the form of the interpolation boundary between overlapping grids. The overlapping grid method is a desirable approach for complex geometry problems. As discussed by Henshaw (1994), the overlapping moving grid method is suitable for moving grid problems, it is more efficient than most other conventional approaches. Interpolation points

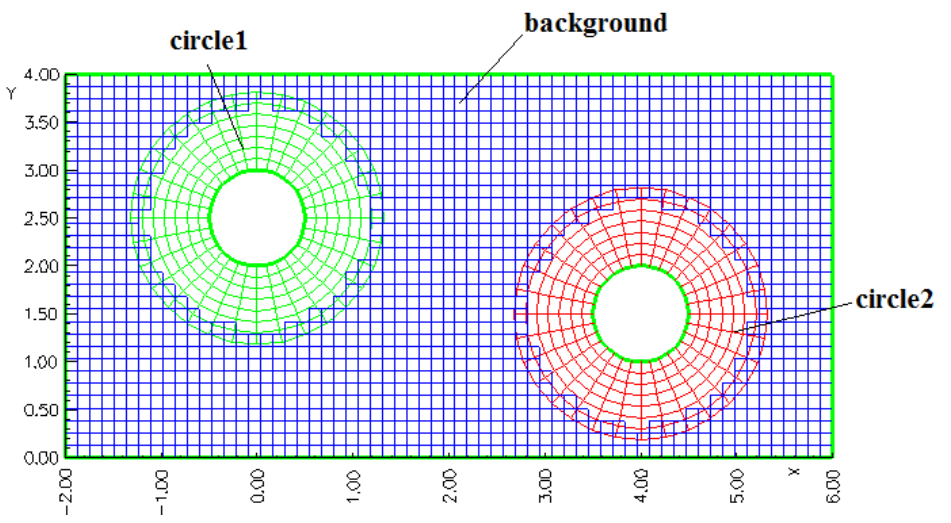

Figure II-3 Overlapping grids.

are located in the overlap region between different grids and are used to couple the solutions. As the body moves, the grid moves with it, meaning that only the interpolation points between grids must be recalculated as opposed to the need to regenerate the whole mesh, as may be necessary with other methods. 
In this dissertation, the particle is modeled as a rigid body with finite volume. The finite particle size is very useful so we can simulate the effect of particle diameter on the electrical signal in the future. Take the two-dimensional problem as an example, the number of degrees of freedom is three (two translational and one rotational). The following Newton-Euler equation is used to describe the particle motion

$$
\frac{d \vec{x}^{b}}{d t}=\vec{v}^{b} \quad M^{b} \frac{d \vec{v}^{b}}{d t}=\vec{F}^{b} \quad \frac{d \vec{h}^{b}}{d t}=\vec{T}^{b}
$$

where $\vec{x}^{b}$ and $\vec{v}^{b}$ are the position and velocity of the center of mass, respectively, $M^{b}$ is the mass of the body, $\vec{F}^{b}$ is the resultant force, and $\vec{h}^{b}$ and $\vec{T}^{b}$ are the angular momentum and resultant torque, respectively, about the center of mass.

At last we consider the numerical method for the electrical potential distribution. Special treatment is needed to solve Laplace's equation because the electrical conductivity is not continuous near the interface between the conductive stream and the low-conductive particle. To deal with the discontinuity in conductivity, the central difference scheme discussed below is used. Eq. (II.4) in the two-dimensional Cartesian coordinates has the following form:

$$
\nabla \bullet(\sigma \nabla \phi)=\frac{\partial\left(\sigma \frac{\partial \phi}{\partial x}\right)}{\partial x}+\frac{\partial\left(\sigma \frac{\partial \phi}{\partial y}\right)}{\partial y}
$$

where the first term on the right hand side is discretized as 


$$
\begin{aligned}
& \frac{\partial\left(\sigma \frac{\partial \phi}{\partial x}\right)}{\partial x}=\frac{\left(\sigma \frac{\partial \phi}{\partial x}\right)_{i+1 / 2, j}-\left(\sigma \frac{\partial \phi}{\partial x}\right)_{i-1 / 2, j}}{d x} \\
& =\frac{\frac{\sigma_{i+1, j}+\sigma_{i, j}}{2} \frac{\phi_{i+1, j}-\phi_{i, j}}{d x}-\frac{\sigma_{i, j}+\sigma_{i-1, j}}{2} \frac{\phi_{i, j}-\phi_{i-1, j}}{d x}}{d x}
\end{aligned}
$$

where $\sigma_{i, j}$ is the electrical conductivity at the grid point $(i, j)$. The second term on the right side of Eq. (II.8) can be discretized similarly. This discretization leads to the system of equations

$$
A \phi_{i, j}+B \phi_{i+1, j}+C \phi_{i-1, j}+D \phi_{i, j+1}+E \phi_{i, j-1}=0
$$

Where

$$
\begin{aligned}
& A=1-w \\
& B=w \frac{\sigma_{i+1, j}+\sigma_{i, j}}{2(d x)^{2}} /\left(\frac{\sigma_{i+1, j}+\sigma_{i, j}}{2(d x)^{2}}+\frac{\sigma_{i, j}+\sigma_{i-1, j}}{2(d x)^{2}}+\frac{\sigma_{i, j+1}+\sigma_{i, j}}{2(d y)^{2}}+\frac{\sigma_{j, i}+\sigma_{i, j-1}}{2(d y)^{2}}\right) \\
& C=w \frac{\sigma_{i, j}+\sigma_{i-1, j}}{2(d x)^{2}} /\left(\frac{\sigma_{i+1, j}+\sigma_{i, j}}{2(d x)^{2}}+\frac{\sigma_{i, j}+\sigma_{i-1, j}}{2(d x)^{2}}+\frac{\sigma_{i, j+1}+\sigma_{i, j}}{2(d y)^{2}}+\frac{\sigma_{j, i}+\sigma_{i, j-1}}{2(d y)^{2}}\right) \\
& D=w \frac{\sigma_{i, j+1}+\sigma_{i, j}}{2(d y)^{2}} /\left(\frac{\sigma_{i+1, j}+\sigma_{i, j}}{2(d x)^{2}}+\frac{\sigma_{i, j}+\sigma_{i-1, j}}{2(d x)^{2}}+\frac{\sigma_{i, j+1}+\sigma_{i, j}}{2(d y)^{2}}+\frac{\sigma_{j, i}+\sigma_{i, j-1}}{2(d y)^{2}}\right) \\
& E=w \frac{\sigma_{i, j}+\sigma_{i, j-1}}{2(d y)^{2}} /\left(\frac{\sigma_{i+1, j}+\sigma_{i, j}}{2(d x)^{2}}+\frac{\sigma_{i, j}+\sigma_{i-1, j}}{2(d x)^{2}}+\frac{\sigma_{i, j+1}+\sigma_{i, j}}{2(d y)^{2}}+\frac{\sigma_{j, i}+\sigma_{i, j-1}}{2(d y)^{2}}\right)
\end{aligned}
$$

Similarly, the difference form in the three dimensional coordinates is

$$
\nabla \bullet(\sigma \nabla \phi)=\frac{\partial\left(\sigma \frac{\partial \phi}{\partial x}\right)}{\partial x}+\frac{\partial\left(\sigma \frac{\partial \phi}{\partial y}\right)}{\partial y}+\frac{\partial\left(\sigma \frac{\partial \phi}{\partial z}\right)}{\partial z}
$$


The terms on the right hand side of (II.11) are discretized as:

$$
\begin{aligned}
& \frac{\partial\left(\sigma \frac{\partial \phi}{\partial x}\right)}{\partial x}=\frac{\left(\sigma_{i+1, j, z}+\sigma_{i, j, z}\right)\left(\phi_{i+1, j, z}-\phi_{i, j, z}\right)-\left(\sigma_{i, j, z}+\sigma_{i-1, j, z}\right)\left(\phi_{i, j}-\phi_{i-1, j, z}\right)}{2(d x)^{2}} \\
& \frac{\partial\left(\sigma \frac{\partial \phi}{\partial y}\right)}{\partial y}=\frac{\left(\sigma_{i, j+1, z}+\sigma_{i, j, z}\right)\left(\phi_{i, j+1, z}-\phi_{i, j, z}\right)-\left(\sigma_{i, j, z}+\sigma_{i, j-1, z}\right)\left(\phi_{i, j, z}-\phi_{i, j-1, z}\right)}{2(d y)^{2}} \\
& \frac{\partial\left(\sigma \frac{\partial \phi}{\partial z}\right)}{\partial z}=\frac{\left(\sigma_{i, j, z+1}+\sigma_{i, j, z}\right)\left(\phi_{i, j, z+1}-\phi_{i, j, z}\right)-\left(\sigma_{i, j, z}+\sigma_{i, j, z-1}\right)\left(\phi_{i, j, z}-\phi_{i, j, z-1}\right)}{2(d z)^{2}}
\end{aligned}
$$

This discretization leads to the system of equations as the following.

$$
a_{0} \phi_{i, j, z}+a_{1} \phi_{i+1, j, z}+a_{2} \phi_{i-1, j, z}+a_{3} \phi_{i, j+1, z}+a_{4} \phi_{i, j-1, z}+a_{5} \phi_{i, j, z+1}+a_{6} \phi_{i, j, z-1}=0
$$

Here

$$
\begin{gathered}
a_{0}=1-w \\
a_{1}=w \frac{\sigma_{i+1, j, z}+\sigma_{i, j, z}}{2(d x)^{2}} /\left(\frac{\sigma_{i+1, j, z}+2 \sigma_{i, j, z}+\sigma_{i-1, j, z}}{2(d x)^{2}}+\frac{\sigma_{i, j+1, z}+2 \sigma_{i, j, z}+\sigma_{i, j-1, z}}{2(d y)^{2}}+\frac{\sigma_{i, j, z+1}+2 \sigma_{i, j, z}+\sigma_{i, j, z-1}}{2(d z)^{2}}\right) \\
a_{2}=w \frac{\sigma_{i, j, z}+\sigma_{i-1, j, z}}{2(d x)^{2}} /\left(\frac{\sigma_{i+1, j, z}+2 \sigma_{i, j, z}+\sigma_{i-1, j, z}}{2(d x)^{2}}+\frac{\sigma_{i, j+1, z}+2 \sigma_{i, j, z}+\sigma_{i, j-1, z}}{2(d y)^{2}}+\frac{\sigma_{i, j, z+1}+2 \sigma_{i, j, z}+\sigma_{i, j, z-1}}{2(d z)^{2}}\right) \\
a_{3}=w \frac{\sigma_{i, j+1, z}+\sigma_{i, j, z}}{2(d y)^{2}} /\left(\frac{\sigma_{i+1, j, z}+2 \sigma_{i, j, z}+\sigma_{i-1, j, z}}{2(d x)^{2}}+\frac{\sigma_{i, j+1, z}+2 \sigma_{i, j, z}+\sigma_{i, j-1, z}}{2(d y)^{2}}+\frac{\sigma_{i, j, z+1}+2 \sigma_{i, j, z}+\sigma_{i, j, z-1}}{2(d z)^{2}}\right) \\
a_{4}=w \frac{\sigma_{i, j, z}+\sigma_{i, j, z-1}}{2(d y)^{2}} /\left(\frac{\sigma_{i+1, j, z}+2 \sigma_{i, j, z}+\sigma_{i-1, j, z}}{2(d x)^{2}}+\frac{\sigma_{i, j+1, z}+2 \sigma_{i, j, z}+\sigma_{i, j-1, z}}{2(d y)^{2}}+\frac{\sigma_{i, j, z+1}+2 \sigma_{i, j, z}+\sigma_{i, j, z-1}}{2(d z)^{2}}\right) \\
a_{5}=w \frac{\sigma_{i, j, z+1}+\sigma_{i, j, z}}{2(d z)^{2}} /\left(\frac{\sigma_{i+1, j, z}+2 \sigma_{i, j, z}+\sigma_{i-1, j, z}}{2(d x)^{2}}+\frac{\sigma_{i, j+1, z}+2 \sigma_{i, j, z}+\sigma_{i, j-1, z}}{2(d y)^{2}}+\frac{\sigma_{i, j, z+1}+2 \sigma_{i, j, z}+\sigma_{i, j, z-1}}{2(d z)^{2}}\right)
\end{gathered}
$$




$$
a_{6}=w \frac{\sigma_{i, j, z}+\sigma_{i, j, z-1}}{2(d z)^{2}} /\left(\frac{\sigma_{i+1, j, z}+2 \sigma_{i, j, z}+\sigma_{i-1, j, z}}{2(d x)^{2}}+\frac{\sigma_{i, j+1, z}+2 \sigma_{i, j, z}+\sigma_{i, j-1, z}}{2(d y)^{2}}+\frac{\sigma_{i, j, z+1}+2 \sigma_{i, j, z}+\sigma_{i, j, z-1}}{2(d z)^{2}}\right)
$$

Here $w$ is the relaxation coefficient. (II.10) and (II.13) can be solved using the successive over relaxation method (Hoffman, 2001). 


\section{Summary}

This chapter introduces the governing equations and simulation methods that are used in this dissertation. The governing equations which are used to solve this problem are Navier-Stokes equations, convection-diffusion equation and Laplace equation. The simulation tool we choose is named Overture which uses overlapping grid to generate the grid. The fluid field is solved by using the Pressure-Poisson method. Once the fluid velocity field is solved, the concentration field can be calculated based on the fluid field. Newton-Euler equation is used to describe the particle motion for solving rigid body movement. At last a new center difference scheme is introduced to solve the Laplace equation. Both two dimensional and three dimensional schemes are illustrated. The equation is solved by a successive over relaxation method based on this difference scheme. 


\section{CODE VALIDATION}

This chapter will validate the solvers we use to confirm their availability in solving these governing equations. The fluid solver is validated by two cases. One is case is steady, laminar fluid flows between infinite fixed parallel plates, and another one is the steady, laminar fluid flows in three dimensional micro channels. The ion concentration field solver is validated by two cases. One is the case that a sample stream is focused by two sheath streams under the uniform velocity distribution; another is the case that a sample stream focused by two sheath streams with three dimensional simulation. The electrical potential solver is validated by comparing the analytical solutions with three different cases which are uniform conductivity distribution along a one dimensional bar, nonuniform conductivity distribution along a one dimensional bar, and uniform conductivity distribution for a two dimensional rectangle domain with coplanar electrodes distribution. 
3.1 Steady, laminar flow between infinite fixed parallel plates

The problem was solved by Munson (2009). It can be considered as two dimensional. The geometry is shown in Figure III-1. The channel has a dimensionless width of $W=1$ and length of $L=4$. The Reynolds number based on the channel height and averaged inlet velocity is 1 . A uniform velocity profile is assigned at the inlet, no-slip boundary conditions are assigned at the top and bottom walls, and zero gauge pressure is assigned at the outlet.

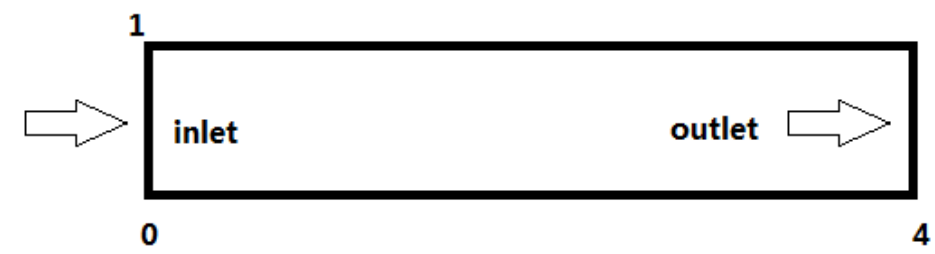

Figure III-1 The schematic of the channel and its dimensions.

Grid sensitivity analysis is performed and the results are shown in Table III-1. The value of Ny denotes the number of grid points used in the y direction of the channel width; the value of $\mathrm{Nx}$ denotes the number of grid points used in the $\mathrm{x}$ direction of the whole channel length. Eight different cases are simulated to test the grid sensitivity. Cross section velocity profiles of the simulation results are compared in Figure III-2.

\begin{tabular}{|c|c|c|c|c|}
\hline $\mathrm{Ny}=25$ & $\mathrm{Nx}=50$ & $\mathrm{Nx}=84$ & $\mathrm{Nx}=100$ & $\mathrm{Nx}=150$ \\
\hline $\mathrm{Ny}=30$ & $\mathrm{Nx}=60$ & $\mathrm{Nx}=100$ & $\mathrm{Nx}=120$ & $\mathrm{Nx}=180$ \\
\hline Ratio $\mathrm{r}=(\mathrm{Nx} / 4) / \mathrm{Ny}$ & 0.5 & 0.84 & 1 & 1.5 \\
\hline
\end{tabular}

Table III-1 Grid sensitivity test for the single channel. 

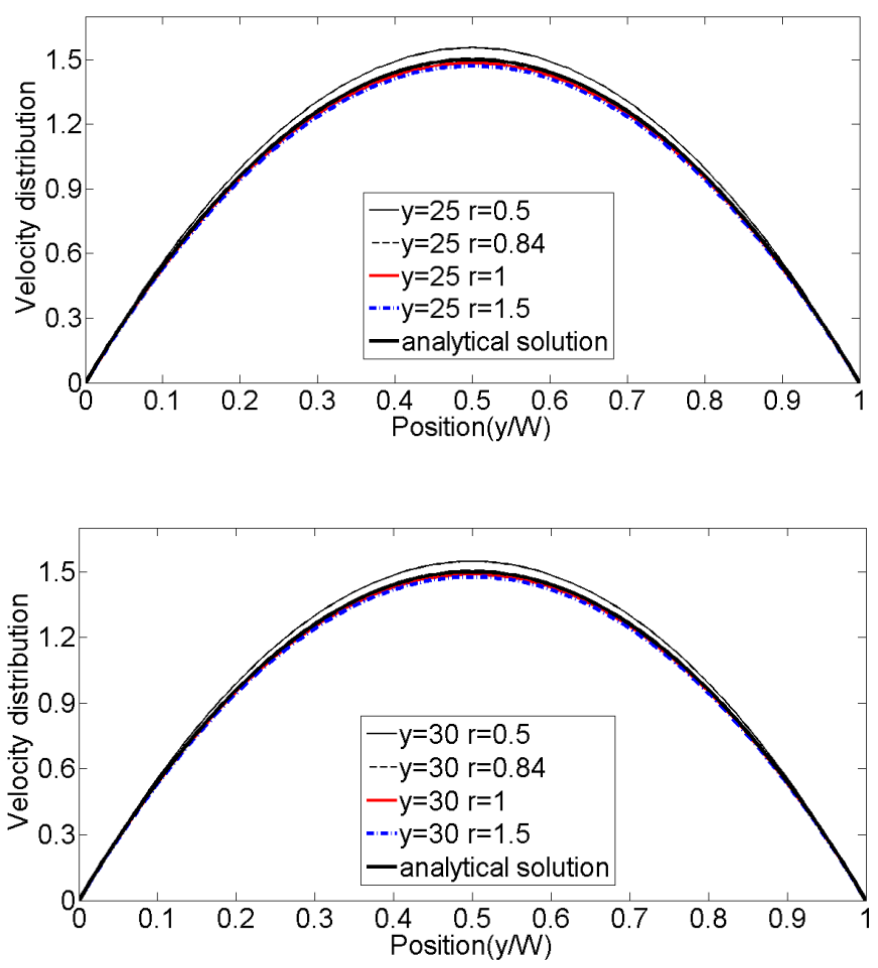

Figure III-2 Comparison of downstream velocity distribution at the fully developed region with the analytical solution.

Figure III-2 shows the grid sensitivity results comparing the analytical solution. We can compare the relative error at the peak of the curves. The analytical solution for the velocity distribution at the fully developed region is parabolic distribution and the max velocity in the axis region is 1.5 times of the mean velocity. For the case of $\mathrm{Ny}=25$, the ratio $\mathrm{r}$ of 0.84 gives the best match with the analytical solution. The case of $\mathrm{Ny}=30$ also gives the best match with a ratio of 0.84 . The max relative errors comparing with the analytical solution about the whole curves are $0.21 \%$ and $0.18 \%$, respectively. This confirms that our solver shows good performance for solving the N-S equations for laminar cases. 


\subsection{Simulation of hydrodynamic focusing}

As shown in Figure III-3, a sample stream and two sheath streams are injected into a two dimensional rectangular channel with the same size as the Figure III-1 shows. Ignoring the viscosity phenomenon at the top and bottom boundary, velocity is uniform across each section, and an analytical solution (Figure I-12) introduced by Wu (2005) shows the sample stream ion concentration distribution. In the simulation we choose of the size as the dimensionless. The width of the sample flow at the inlet is set to 0.2 of the channel width, the concentration of the sample flow at the inlet is set to 1 , the concentration of the

sheath flow at the inlet is 0 , The Reynolds number is set as land the Peclet number is 2000. Three different grids are tested. Table III-2 shows the error in the peak concentration between the numerical and analytical solutions at the line of $x=3$.

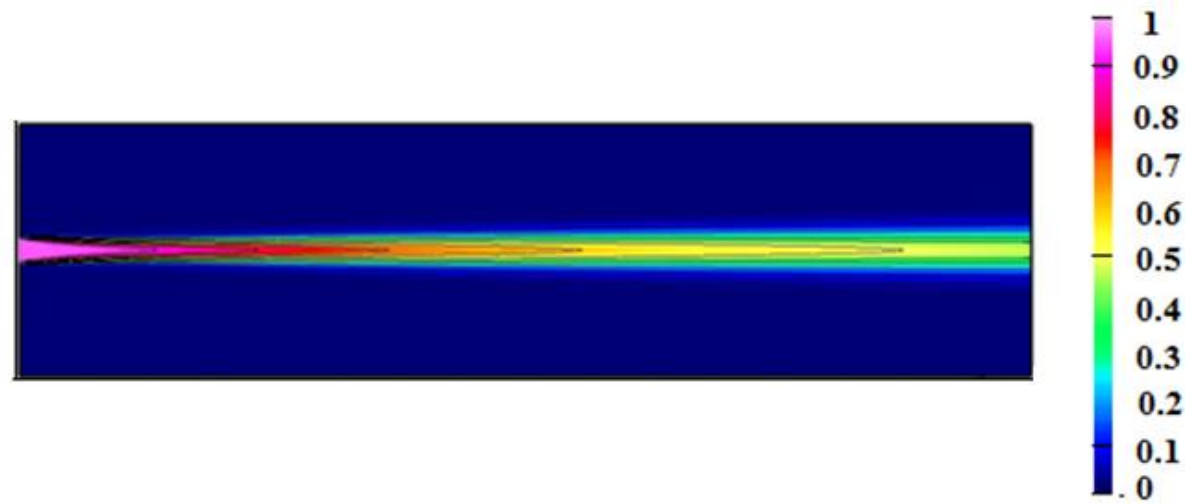

Figure III-3 Concentration for a sample stream injected into a 2D channel.

From Table III-2 we can see that the solution is more sensitive to the grid resolution in the $y$ direction than in the $x$ direction. The result of $\mathrm{Ny}=201, \mathrm{Nx}=201$ shows that the grid points density that 201 points for distance 1 on the vertical direction can capture the 
concentration. The max relative error for the whole simulated curve is $1.6 \%$ and this grid sensitivity is used in this paper for capturing the concentration value. Figure III-4 shows the concentration distribution at the cross section of $x=1,2$, and 3 . The ion concentration distribution becomes smoother as the cross section moves towards downstream.

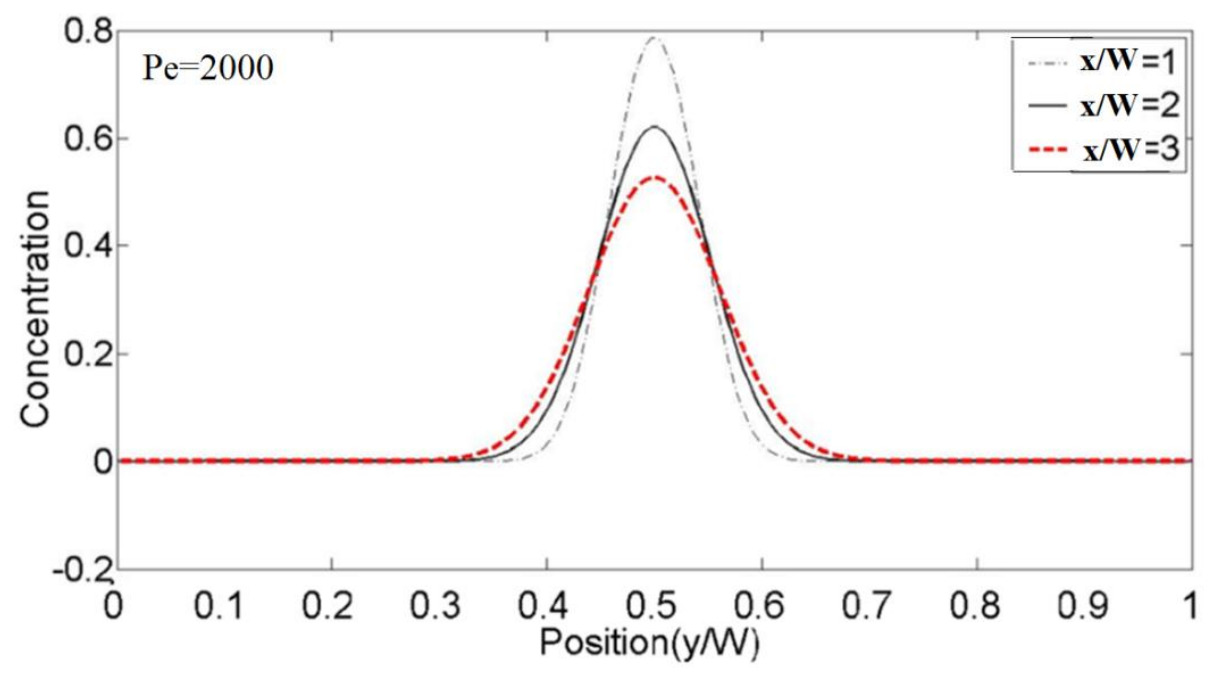

Figure III-4 Simulation results of ion concentration distribution at different downstream locations.

\begin{tabular}{|c|c|c|c|}
\hline $\mathrm{x}=3$ & $\mathrm{Ny}=101$ & $\mathrm{Ny}=201$ & $\mathrm{Ny}=301$ \\
\hline $\mathrm{Nx}=200$ & $6.71 \%$ & $1.60 \%$ & $0.63 \%$ \\
\hline $\mathrm{Nx}=400$ & $6.72 \%$ & $1.61 \%$ & $0.64 \%$ \\
\hline
\end{tabular}

Table III-2 Relative max error in concentration using different grid spacing. 
3.3 Velocity and ion concentration distribution on 3D micro channel

White (1991) introduced the analytical solution for the velocity distribution at the fully developed region when the flow is laminar and the cross section of the channel is rectangular. The range of the width and length is $-a \leq y \leq a$ and $-b \leq z \leq b$. So the velocity $u(y, z)$ at any point $(y, z)$ in the cross section of the fully developed region can be calculated as

$$
\begin{gathered}
u(y, z)=\frac{16 a^{2}}{\mu \pi^{3}}\left(-\frac{d p}{d x}\right) \sum_{i=1,3,5, \ldots}^{\infty}(-1)^{(i-1) / 2}\left[1-\frac{\cosh (i \pi z / 2 a)}{\cosh (i \pi b / 2 a)}\right] \times \frac{\cos (i \pi y / 2 a)}{i^{3}} \\
Q=\frac{4 b a^{3}}{3 \mu}\left(-\frac{d p}{d x}\right)\left[1-\frac{192 a}{\pi^{5} b} \sum_{i=1,3,5, \ldots}^{\infty} \frac{\tanh (i \pi b / 2 a)}{i^{5}}\right]
\end{gathered}
$$

Where $\mu$ is the viscosity of the fluid, $\frac{d p}{d x}$ is the pressure drop gradient at the flow direction and $Q$ is the volume flow rate of the fluid. Here we compare our dimensionless numerical simulation results with the analytical solution to validate them. The simulation domain size is shown in Figure III-5.

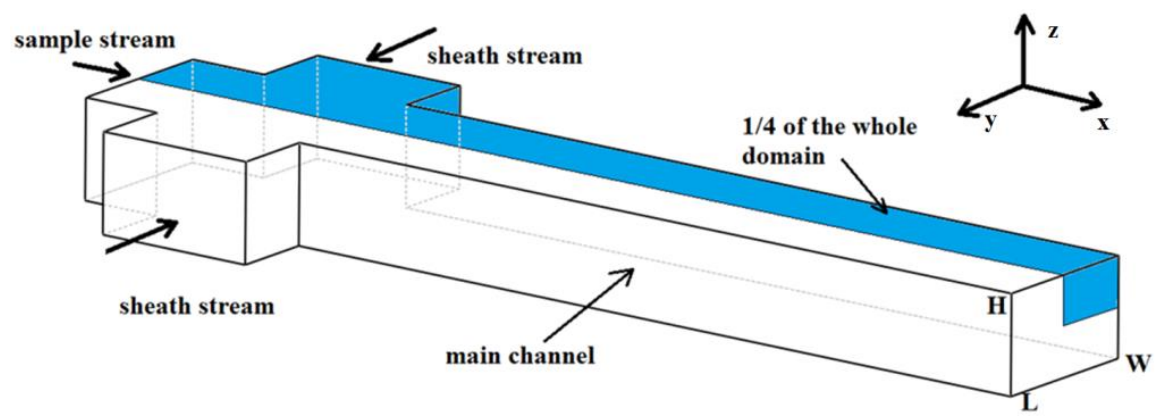

Figure III-5 The simulation domain of the three dimensional micro channel. 
The size of the micro channel is: length $L=6.5$, width of the each inlet $W=1$ and the height of the micro channel $H=0.6$. The Reynolds number is 10 and the Peclet number is 200. Here we use $Q_{\text {sun }}$ to represent the total fluid stream flow rate, $Q_{\text {sample }}$ to present the sample stream flow rate and $Q_{\text {sheath }}$ to present the sheath stream flow rate. Our sample stream flow rates are defined as $Q_{\text {sample }} / Q_{\text {sum }}=0.2$. The concentration of the sample stream at the inlet is 1 while the concentration of the sheath stream at the inlet is 0.1 . Similar as before, we use Nx to present the grid points in the $\mathrm{x}$ coordinate direction of the main channel, use Ny to present the grid points in the y coordinate direction of the main channel and use $\mathrm{Nz}$ to present the grid points in the $\mathrm{z}$ coordinate direction of the main channel. Three cases (Table III-3) are processed to test for a grid independent solution.

\begin{tabular}{|c|c|}
\hline Case & Grid points variation (main channel) \\
\hline No.1 & $\mathrm{Nx}=433 \mathrm{Ny}=81 \mathrm{Nz}=17$ \\
\hline No.2 & $\mathrm{Nx}=433 \mathrm{Ny}=97 \mathrm{Nz}=17$ \\
\hline No.3 & $\mathrm{Nx}=537 \mathrm{Ny}=101 \mathrm{Nz}=21$ \\
\hline
\end{tabular}

\section{Table III-3 Two different grid density cases.}

The velocity distribution on the line $(x / W=3, y / W=0.5)$ is compared with the analytical solution on Figure III-6. We can see that the numerical simulation results match the analytical solution well; the maximum relative error is no more than $1 \%$ comparing with the analytical solution with the whole simulation curves. 


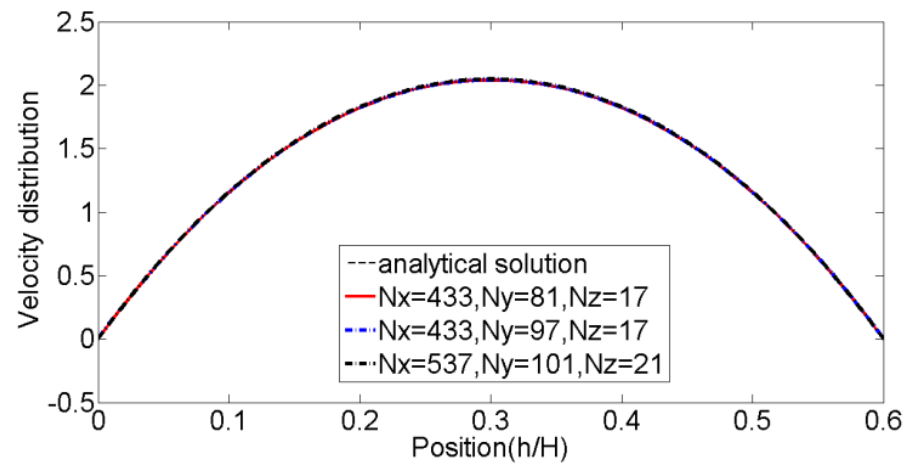

Figure III-6 Analytical solution about the velocity distribution and simulation results.

Then we check the concentration distribution $(x / W=3, h / H=0.5)$ which is shown in Figure III-7. The results show that these solution curves nearly coincide with each other; the max relative error of these three results on the whole curves is no more than $0.32 \%$.
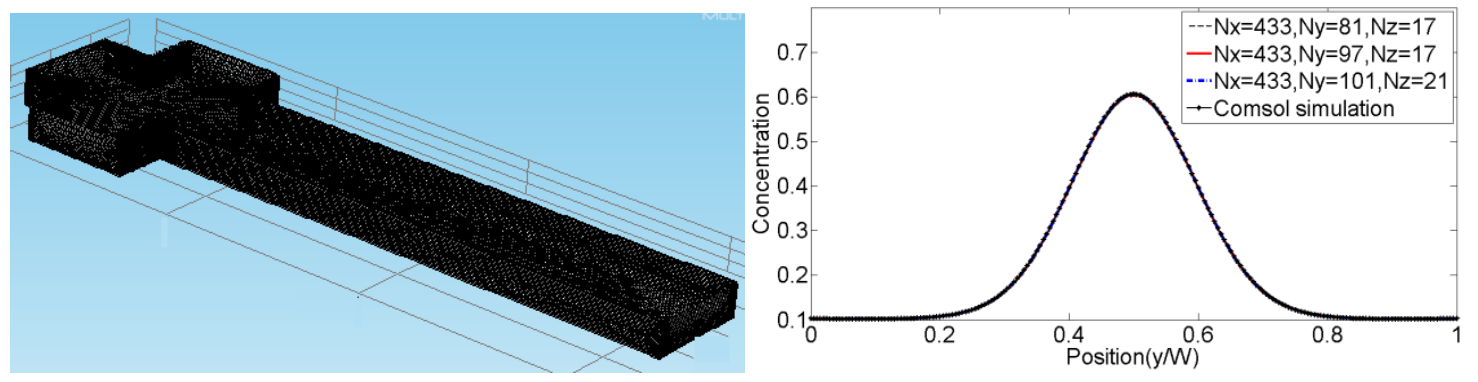

Figure III-7 Ion concentration distribution. Left one is the mesh (half of the domain) by Comsol, right one is the simulation results including Overture and Comsol.

The same simulation is processed again by using commercial software Comsol (edition v4.4). We can compare the simulation results from the dissertation source code and Comsol microfluidic toolbox at the same location $(x / W=3, h / H=0.5)$. It is shown in 
too. We can see that the simulation results from our source code matches the Comsol simulation results well with the maximum relative error is no more than $0.8 \%$.

The following cases will simulate another situation and compare it with the analytical solution. Consider a cylinder with diameter $d$ and length $L$ as the Figure III- 8 shows. The velocity distribution from the sample stream coming at the left is shown in Eq.(III.3).

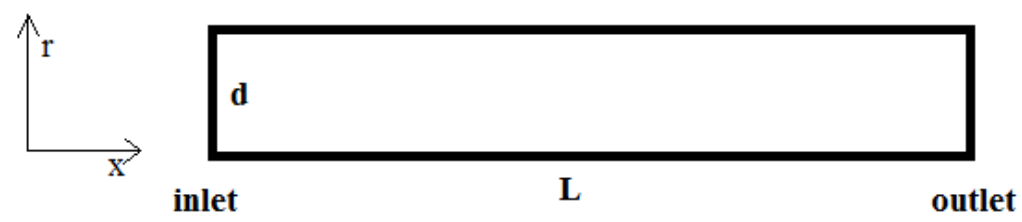

Figure III-8 the schematic of the cylinder.

$$
u(r)=2 \bar{u}\left(1-\frac{4 r^{2}}{d^{2}}\right)
$$

Here $\bar{u}$ is the mean velocity of the sample stream. We can solve the concentration distribution by the following

$$
\frac{\partial C}{\partial t}+u \frac{\partial C}{\partial x}=D\left[\frac{\partial^{2} C}{\partial x^{2}}+\frac{1}{r} \frac{\partial}{\partial r}\left(r \frac{\partial C}{\partial r}\right)\right]
$$

Here $\mathrm{C}$ is the concentration and $\mathrm{D}$ is the diffusion coefficient. The boundary conditions and initial conditions will be

$$
\begin{aligned}
& C(x, d / 2, t)=0 \\
& C(x, r, 0)=1 \\
& C(0, r, t)=0 \quad t>0
\end{aligned}
$$


Then we compared the numerical solutions under the assuming that when the molecular diffusivity was set to zero with the analytical results. The mixing cup concentration $\bar{C}(x, t)$ is defined as the following

$$
\bar{C}(x, t)=\frac{\int_{0}^{d / 2} 2 \pi r u(r) C(x, r, t) d r}{\int_{0}^{d / 2} 2 \pi r u(r) d r}
$$

Based on Gomes et al. (1993), for the outlet, the ratio of the mixing cup concentration at time $t$ and the concentration at the beginning can be calculated as

$$
\begin{aligned}
C_{\text {ratio }}=\frac{\bar{C}(L, t)}{\bar{C}(L, 0)}=1 \quad t \in\left[0, \frac{L}{2 \bar{u}}\right] \\
C_{\text {ratio }}=\frac{\bar{C}(L, t)}{\bar{C}(L, 0)}=\frac{L^{2}}{4(\bar{u} t)^{2}} \quad t \in\left[\frac{L}{2 \bar{u}},+\infty\right]
\end{aligned}
$$

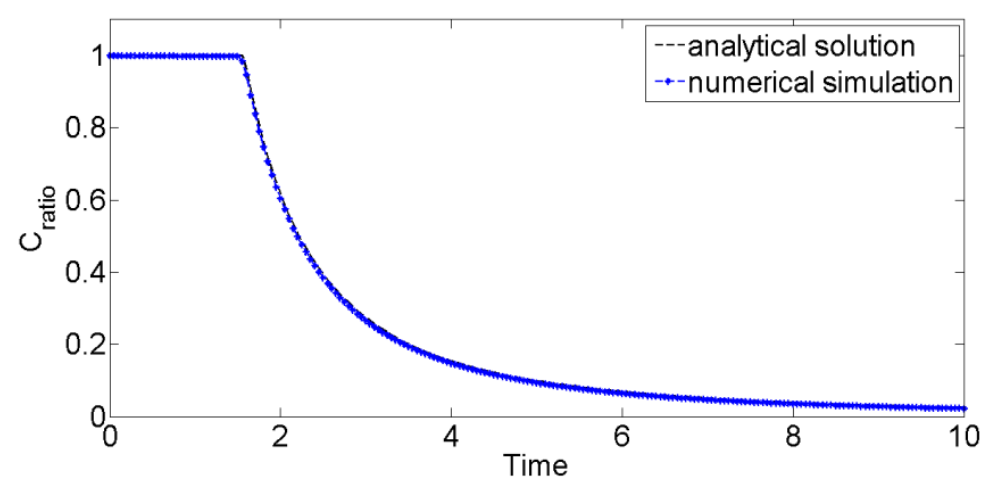

Figure III-9 Comparison with the analytical and numerical solution for the convection only situation. 
For the code variation, we choose the diameter of the cylinder is 1 , the length of the pipe is 100 and $L / \bar{u}$ is 3.14. Figure III-9 shows the comparison for the analytical solution and numerical solution. The similar work can be found in Gomes et al. (1993). It shows that these two curves match each other very well.

Based on the simulation results above, our Overture source codes can simulate the convection-diffusion phenomenon with the acceptable accuracy.

\subsection{Electrical potential distribution}

Three test cases are used to validate the electrical potential calculation. First we consider the electric potential distribution along a one-dimensional conductive bar. The onedimensional bar is shown in Figure III-10. The bar has a dimensionless length of $L=1$. Two test cases are considered. In the first one, the unit-length bar has a uniform, dimensionless electrical conductivity of 1 . In the second case, the electrical conductivity is 0.1 between $0.222<x<0.322$ but is 1 everywhere else. An analytical solution exists in both cases and they are shown on the right side of Figure III-10. Figure III-11 compares the simulated and analytical solutions. In both cases the numerical results match the analytical ones. 


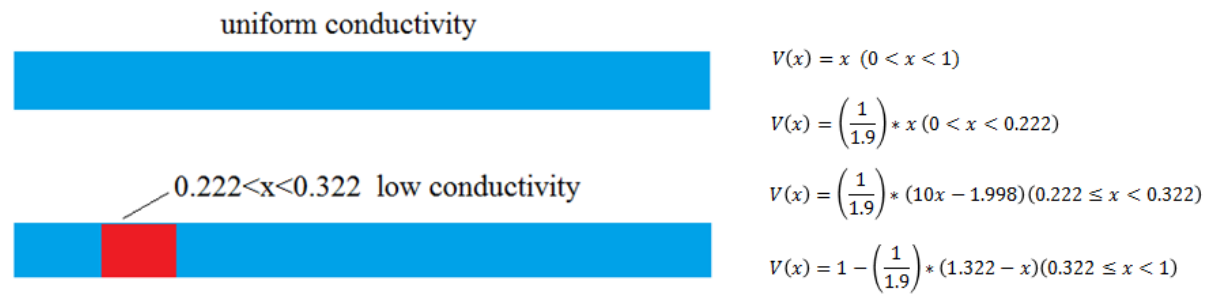

Figure III-10 Schematic of the one-dimensional conductive bars. Top: onedimensional bar with uniform electrical conductivity; bottom: one-dimensional bar with non-uniform conductivity, the red region has low conductivity. $V(x)$ is the potential distribution along the one dimensional bar.
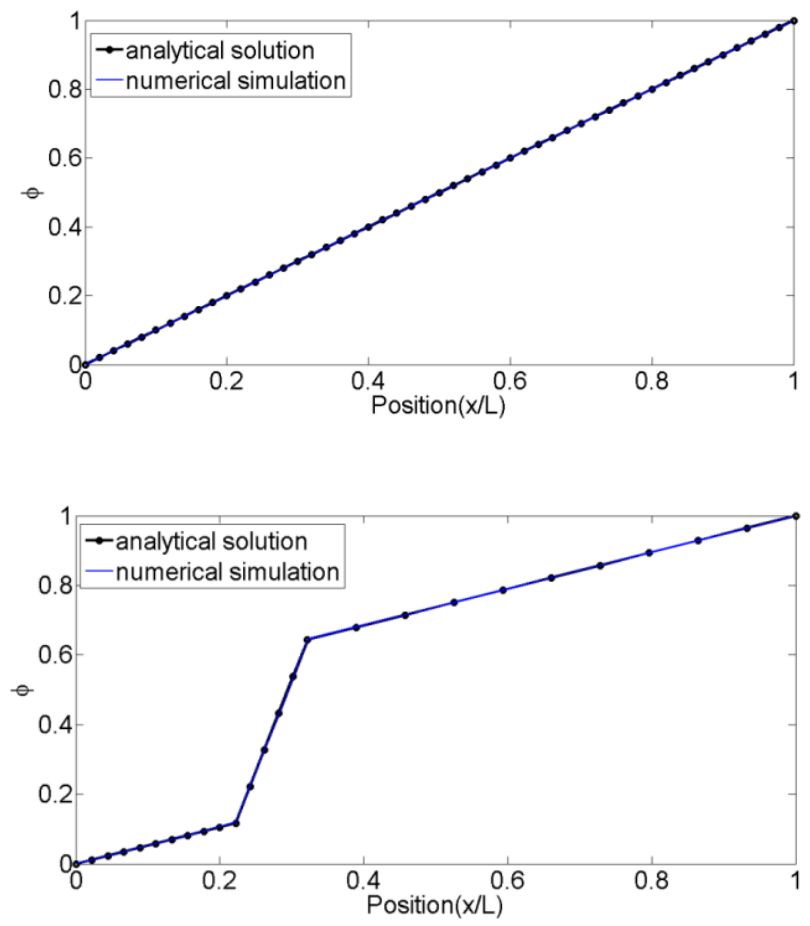

Figure III-11 Comparison of electric potential distribution along a bar with different conductivities. Top one is the case with uniform conductivity, bottom one is the case with non-uniform conductivity. 
Thirdly, we study the electric potential distribution in a two-dimensional (2D) field. The 2D channel has a length of $L$ and height of $H$. A uniform electrical conductivity distribution is given to the channel region. Two electrodes of equal size are mounted on the bottom surface. The remaining boundary regions are set as insulating boundaries (Figure III-13). An analytical solution was obtained by Sun et al. (2007)). They used a mathematic mapping method named Schwarz-Christoffel Mapping (Trefethen (1980), Chaudhry and Schinzinger (1992), Delillo and Kropf (2011)). The calculated domain (infinite domain with limited height) and its analytical formula is shown below
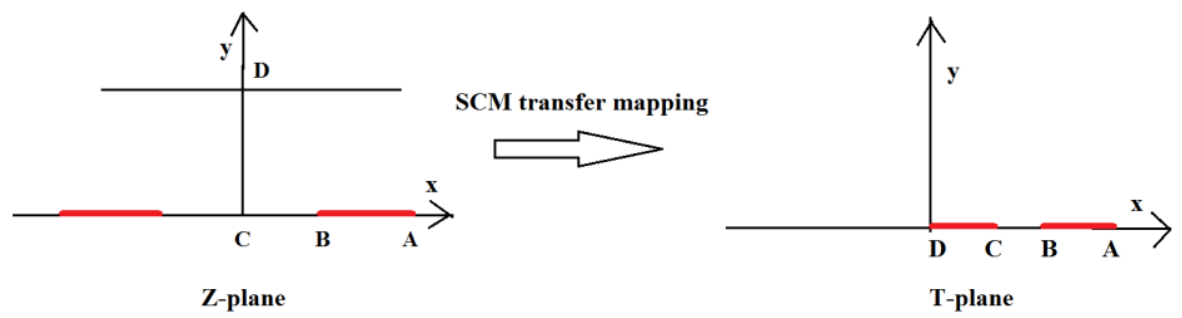

Figure III-12 The coplanar electrodes distribution and SCM transfer mapping (Sun et al.(2007)).

$$
E_{2 b}=\overline{\frac{\pi V}{2 h_{b} K\left(k_{b}\right)}\left(\frac{T_{A}-T_{C}}{T-T_{A}}\right)^{1 / 2}\left(\frac{T_{B}-T_{D}}{T-T_{B}}\right)^{1 / 2}}
$$

Here $E_{2 b}$ is the electrical field distribution, $T_{i} \quad i=A, B, C, D$ is the coordinate value of the domain point $\mathrm{A}, \mathrm{B}, \mathrm{C}$ and $\mathrm{D}$ in T-plane after the SCM transfer mapping. $h_{b}$ is the finite height of the domain in Z-plane. $k_{b}$ is the cell constant per unit width of the microfluidic channel (it is defined in the reference). $K\left(k_{b}\right)$ is the complete elliptic integral of the first 
kind. Here we compares the numerical and analytical results along line $O B($ length $=0.1)$. It is noted that the analytical solution is obtained for an infinitely long channel and simulations are based on finite length channels. Figure III-14 shows that the agreement between the analytical and numerical results is very good for all the three situations (max relative error for the whole simulation curves are $1.1 \%$ ); the boundary has no significant effect on our results. The calculated potential distribution is shown in Figure III-15.

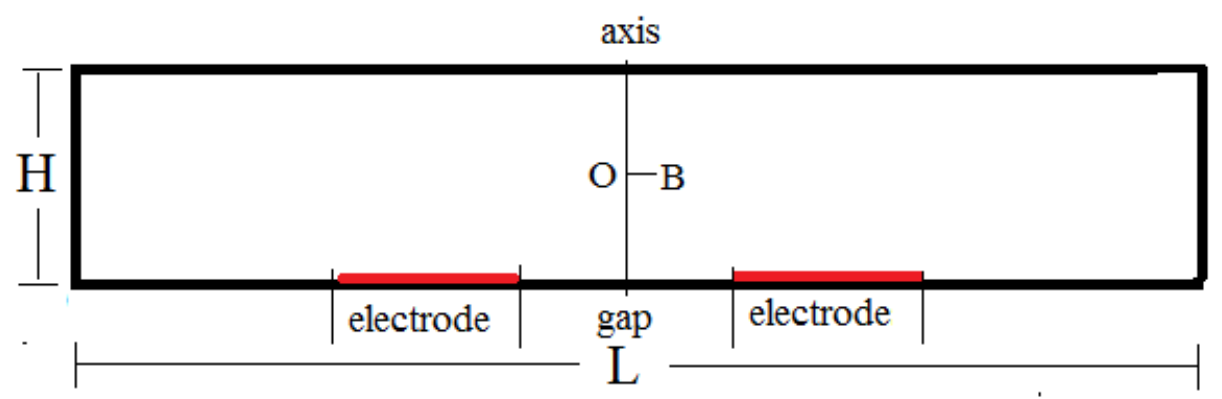

Figure III-13 Schematic of a finite length channel with electrodes mounted on the bottom.

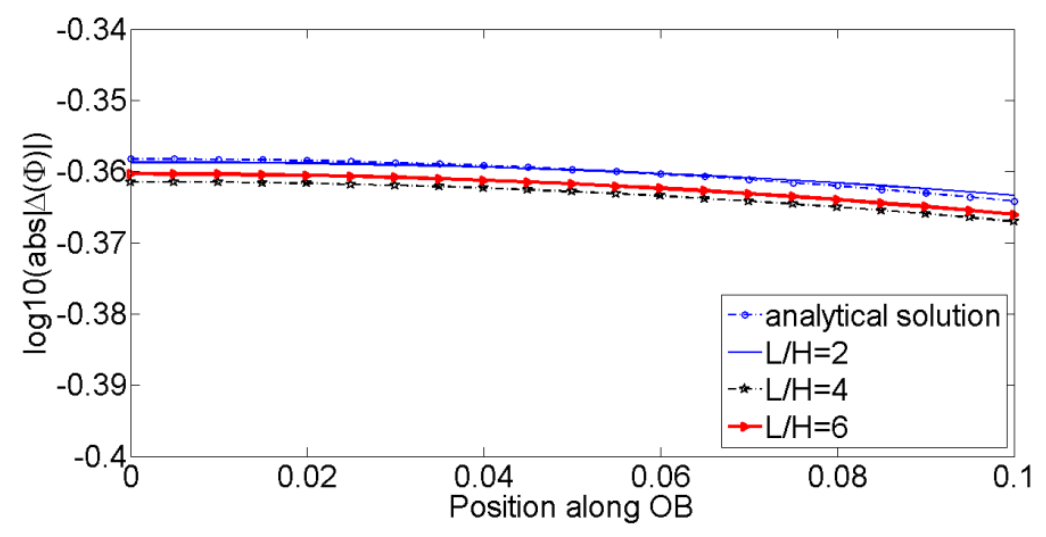

Figure III-14 Comparison of the electrical magnitude between numerical and analytical approaches. 


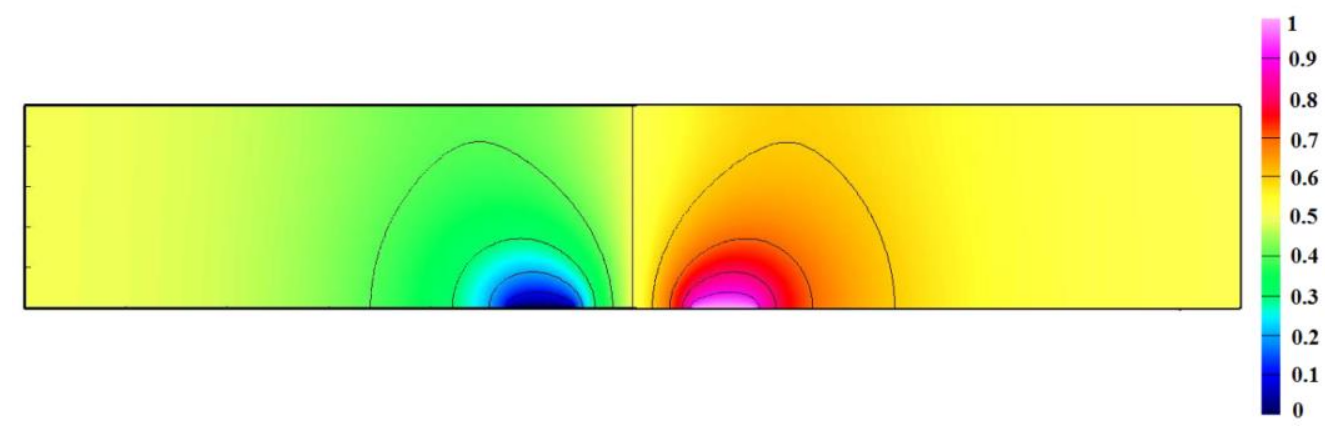

Figure III-15 Potential distribution in a 2D channel. 


\section{Summary}

This chapter validates our solvers by simulating cases under several different situations. The fluid and ion concentration solvers are validated by the cases of steady, laminar flow between infinite fixed parallel plates, hydrodynamic focusing with uniform velocity distribution and hydrodynamic focusing under the three dimensional simulation. The velocity simulation result is compared with the analytical solution about its distribution at the fully developed region. The results show that they match the analytical solution with acceptable errors. The ion concentration solution is compared either with the analytical solution or with the solution under the grid density variation. Both the results show that the ion concentration solver can capture the concentration distribution correctly and our results are grid sensitivity independent. At last the electrical potential solver is validated by comparing the analytical solutions under three different cases. All the numerical solutions are compared with the analytical solution and the results show that our electrical potential solver can calculate the potential distribution with the available errors. 


\section{SIMULATION FOR HYDRODYNAMIC FOCUSING}

The chapter will show the simulation results for the hydrodynamic focusing phenomenon. The first part of the simulation results includes the ion concentration distribution in the micro channel. Then the impacts of the diffusion coefficient, the sample/sheath stream flow rates ratio, and the asymmetric flow situation are discussed. Both two dimensional and three dimensional simulation cases are used. The second part of this chapter focuses on the particle motion under the impact of the hydrodynamic focusing. The particle motion including one particle, two particles and three particles are studied. The impacts of the sample stream flow rate variation under the hydrodynamic focusing application are investigated. 


\subsection{Ion diffusion phenomenon}

The physical situations are shown below: two sheath flows are injected from the top and bottom inlets, and the sample flow is injected from the horizontal inlet. For convenience here we use dimensionless parameters. The total length of the micro channel is $L=9$ and the width is $W=1$, the three inlets have the same width of $W=1$. We use $Q_{\text {sum }}$ as the total fluid stream flow rate, $Q_{\text {sample }}$ as the sample stream flow rate and $Q_{\text {sheath }}$ as the rate of each sheath stream (at first we just consider the symmetric situation. It means the rate of each sheath stream is the same). In this simulation, the sample stream flow rate is $Q_{\text {sample }} / Q_{\text {sum }}=0.2$. The Reynolds number based on the averaged flow velocity in the main downstream channel is one. The Peclet number is 2000 . The sample stream has the relatively high concentration which is 1 while the concentration of the sheath stream has the relatively low concentration which is 0.1 . Figure $0-1$ shows the concentration contours after the flow becomes steady. The sample flow is hydrodynamically focused in the main channel by the two sheath flows. Its width gradually increases due to the slow molecular diffusion.

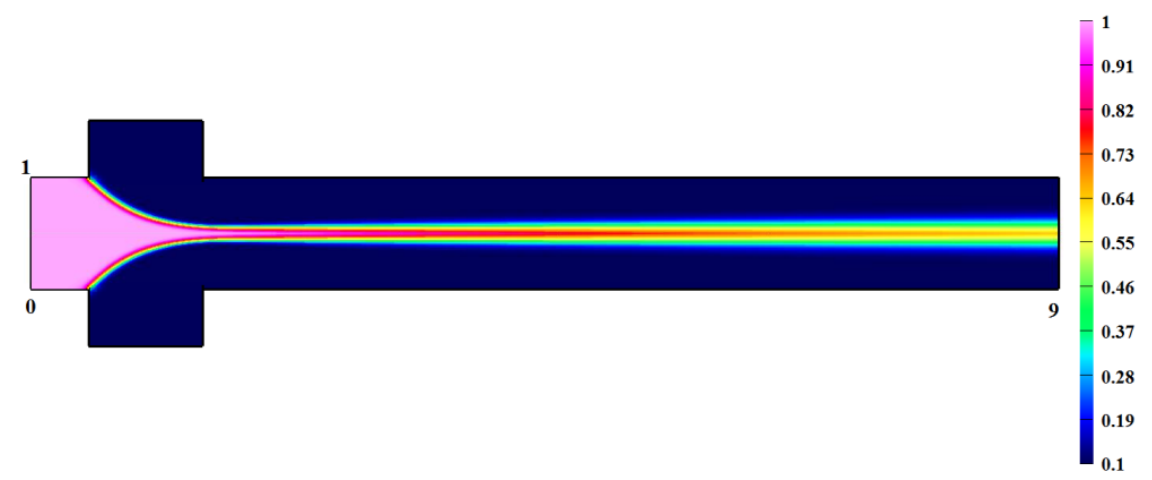

Figure 0-1 Sample flow concentration due to hydrodynamic focusing. 


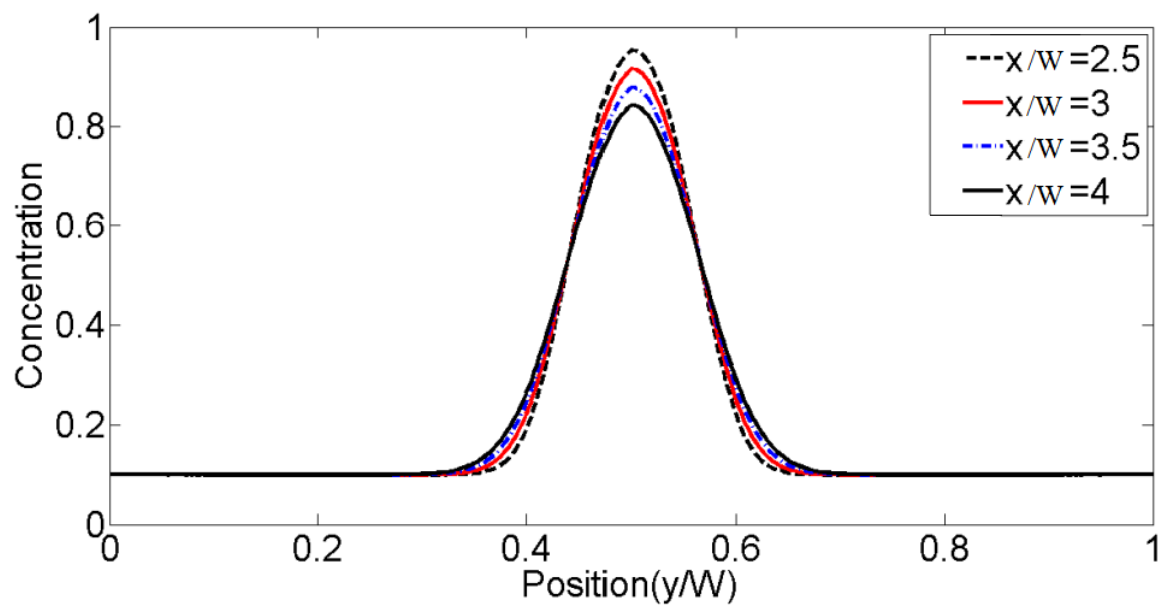

Figure 0-2 The concentration profiles at different locations.

Figure 0-2 shows the concentration profile at downstream distances of $\mathrm{x} / \mathrm{W}=2.5,3,3.5$ and 4. The peak value decreases and the bell shaped concentration profiles become wider as the sample flow moves downstream due to the molecular diffusion.

\subsection{The impacts of Reynolds number}

Figure 0-3 compares the concentration distribution along the center line for the cases of different Reynolds number by fixing the Peclet number. In all the three cases the sample flow rate is $Q_{\text {sample }} / Q_{\text {sum }}=0.2$. The Peclet number is fixed on 2000 . We can see that there is just a slight variation of the ion concentration distribution (the max relative error at the end of the vertex is less than $1 \%$ ).

Also Figure 0-3 compares the concentration profiles at $\mathrm{x} / \mathrm{W}=4.5$ for the three cases. It shows the same tendency that the variation of the Reynolds number from 1 to 10 has nearly no influence to the ion concentration distribution (the max relative error at the peak is less than $1 \%$ ). 

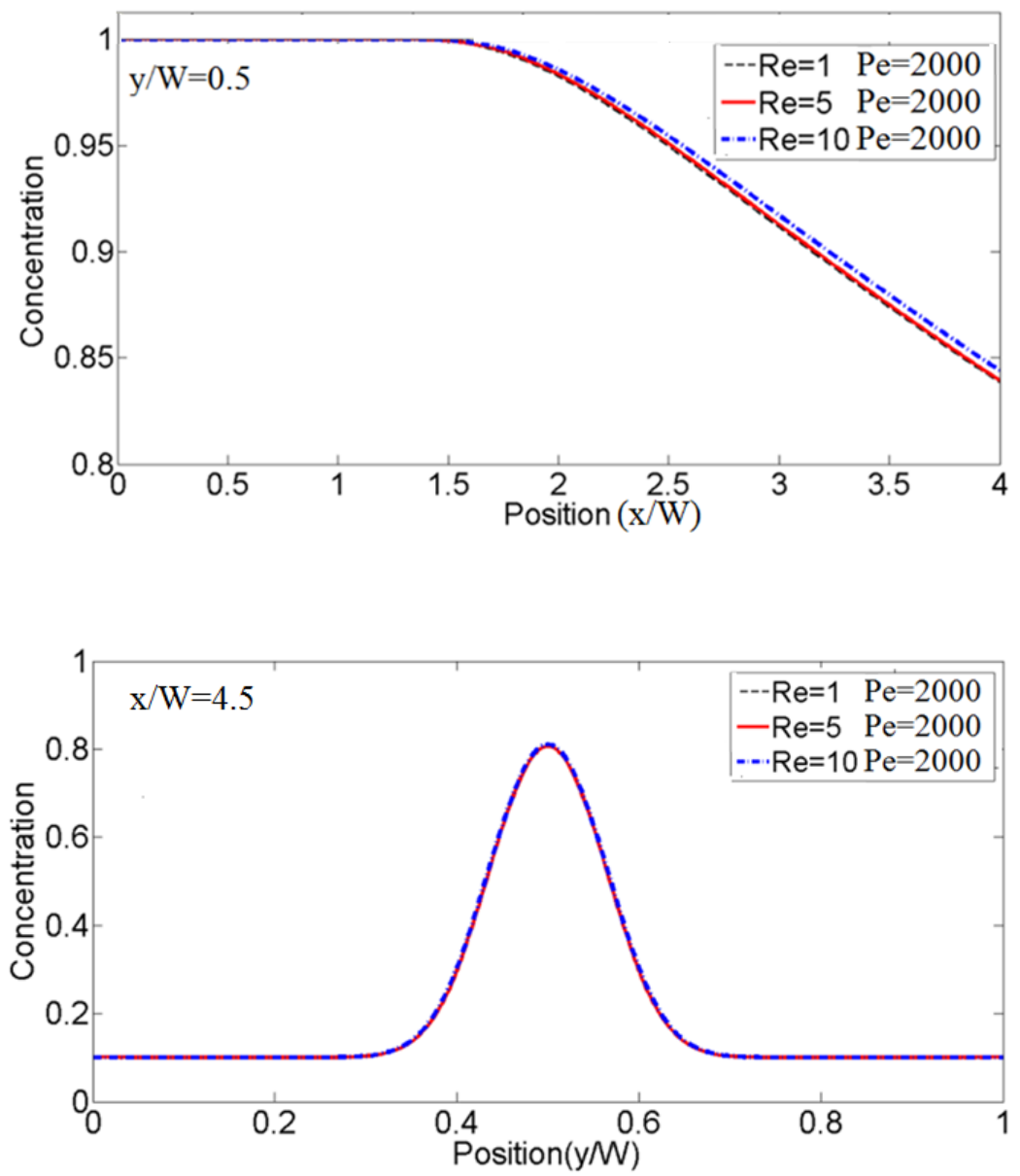

\section{Figure 0-3 The concentration profiles at different Reynolds numbers by fixing Peclet number.}

Figure 0-4 compares the concentration distribution along the center line for the cases of different Reynolds number by fixing the Schmidt number. In all the three cases the sample flow rate is $Q_{\text {sample }} / Q_{\text {sum }}=0.2$. The Schmidt number is fixed on 1000. It shows that smaller Reynolds number can get lower concentration distribution. Also Figure 0-4 compares the concentration profiles at $\mathrm{x} / \mathrm{W}=4.5$ for the three cases. It shows that smaller Reynolds number can get smoother concentration distribution. This is because under the 
condition of same Schmidt number, smaller Reynolds number can get larger diffusion coefficient, and that can make the concentration distribution smoother.
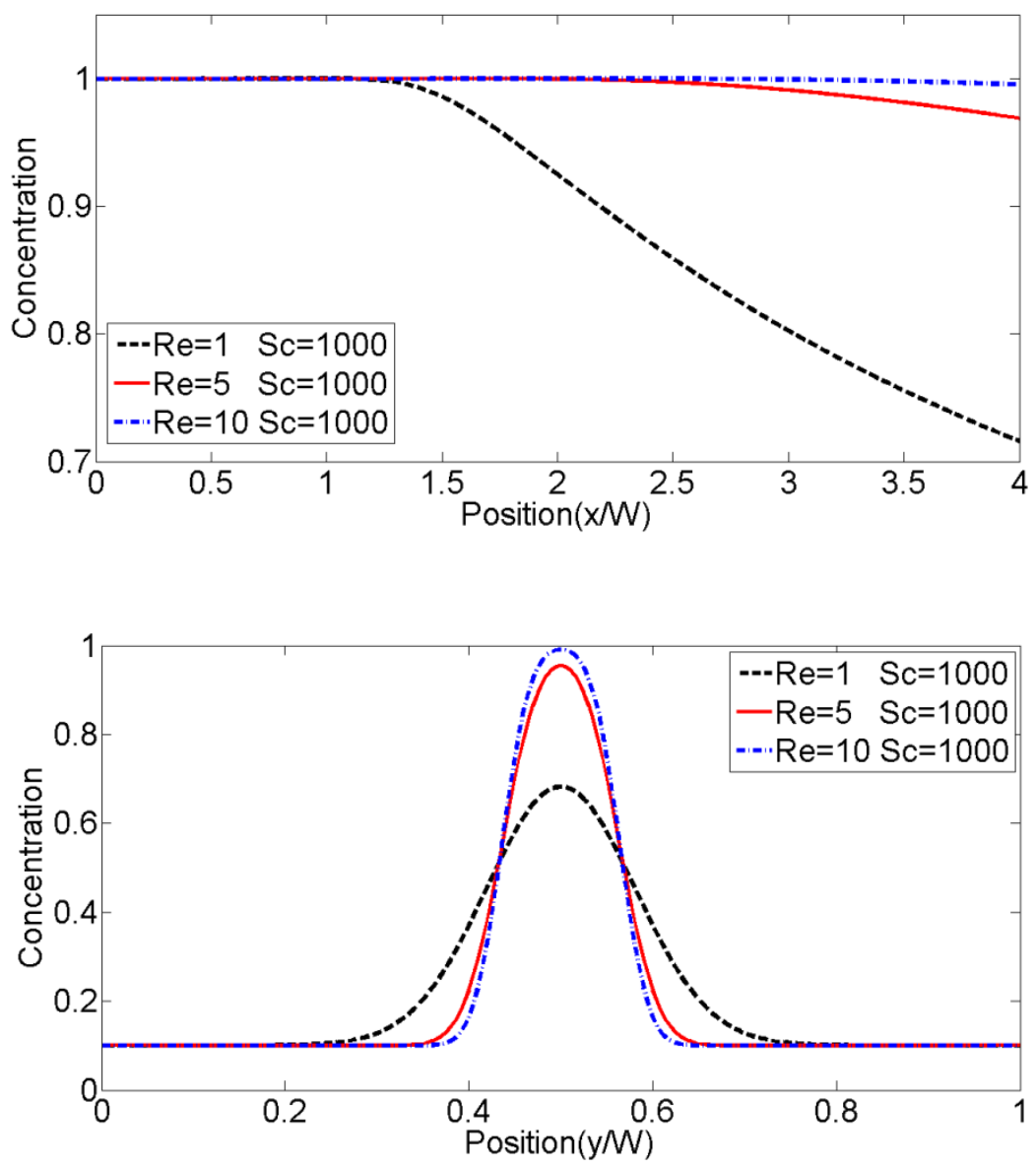

\section{Figure 0-4 The concentration profiles at different Reynolds numbers by fixing} Schmidt number.

\subsection{The impacts of Peclet number}

Figure 0-5 compares the concentration distribution along the center line for different Peclet numbers. In all four cases the sample flow rate is $Q_{\text {sample }} / Q_{\text {sum }}=0.2$. We keep the Reynolds number as 1 and then change the Peclet number $(P e=4000,2000,1333,1000)$. 
We can see that a smaller Peclet number means a higher molecular diffusivity and that leads to a quicker drop in the concentration along the center line. Also Figure 0-5 compares the concentration profiles at $\mathrm{x} / \mathrm{W}=4.5$. A higher Peclet number flow has a more confined distribution while a lower Peclet number flow has a flatter distribution.
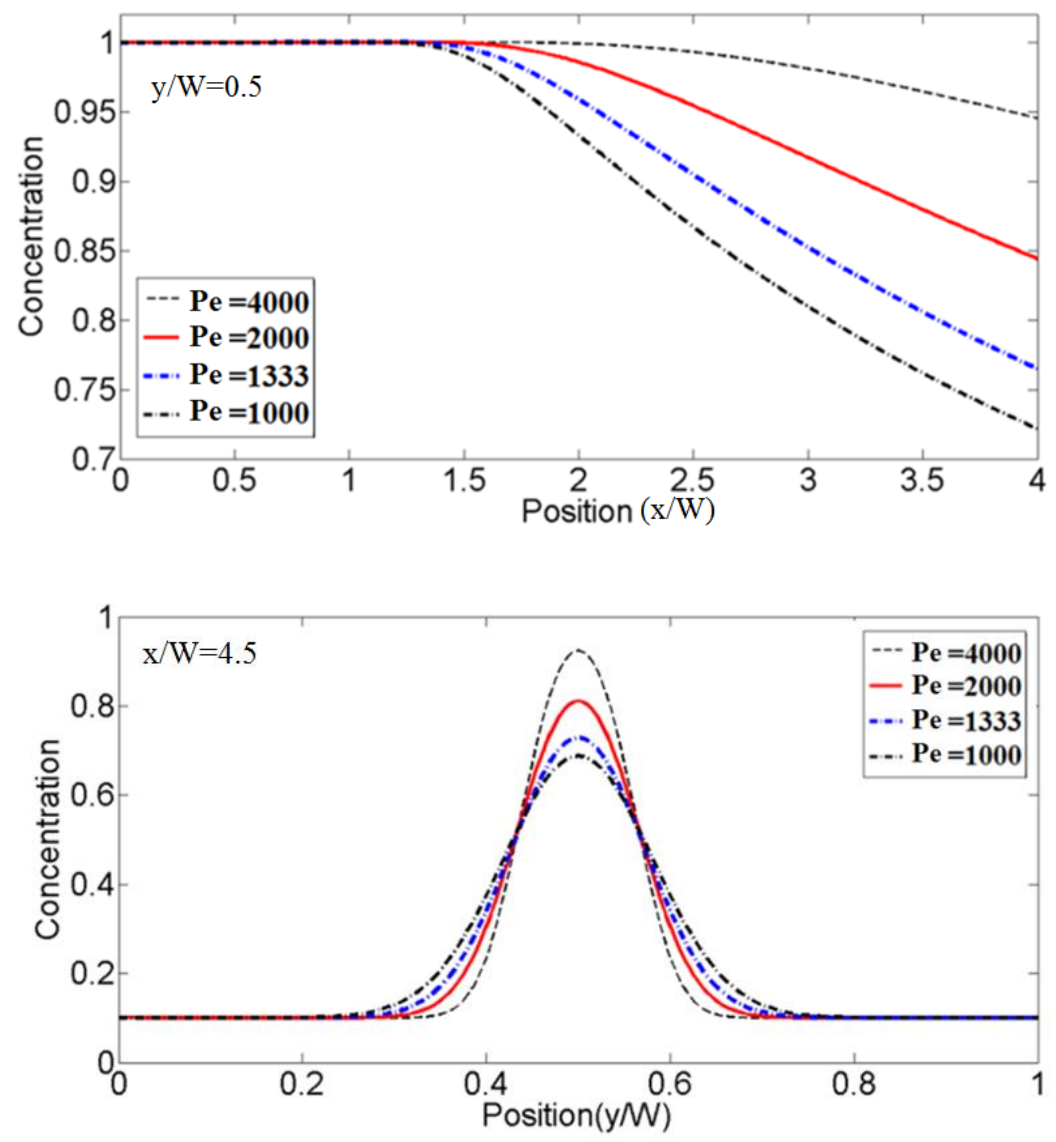

Figure 0-5 The concentration profiles at different $\mathrm{Pe}$ with $\mathrm{Re}=1$.

4.4 The impacts of relative sample and sheath flow rate

The relative flow rate is another important factor to determine the performance of hydrodynamic focusing. To illustrate the salient features of their model we first consider a case in which the two sheath flows have the same flow rate. The microfluidic 
configuration is shown in Figure $0-1$ in which the top and bottom inlets have the same width values. In this dissertation five different sample flow rates are tested ( $\left.Q_{\text {sample }} / Q_{\text {sum }}=0.1,0.15,0.2,0.25,0.3\right)$. To maintain the same Reynolds number, we adjust the sheath flow rate to keep the total flow rate constant. Figure $0-6$ shows the concentration of the sample stream along the center line. These profiles show that as the relative sample rate to the total flow rate decreases, the concentration along the center line decreases. Figure 0-7 shows the concentration profile at $\mathrm{x} / \mathrm{W}=4.5$. The peak concentration decreases as the relative flow rate of the sample stream decreases. This is because as sample stream flow rate decreases, less sample ions move into the channel at the same time period, leading to the low concentration.

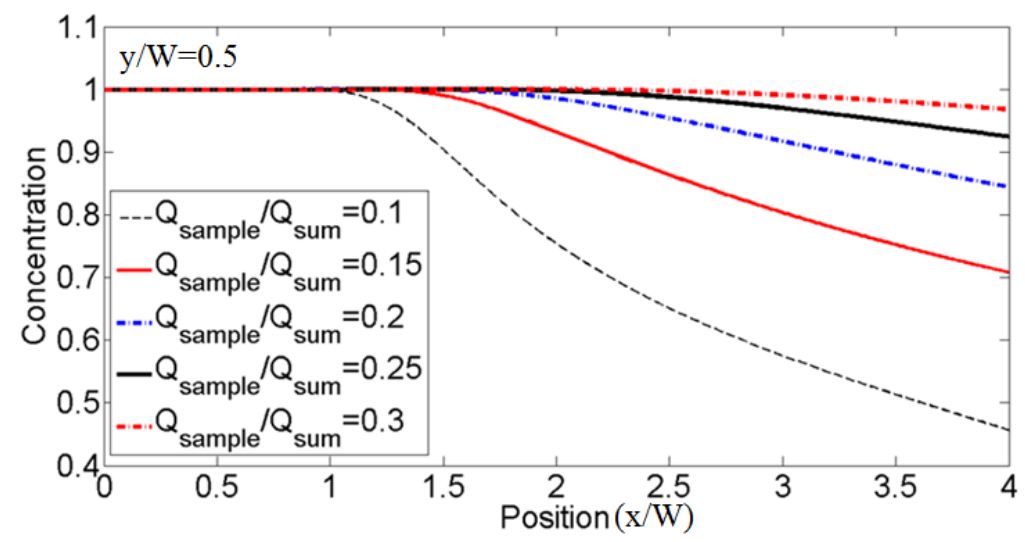

Figure 0-6 Concentration along the center line for varied relative flow rates. 


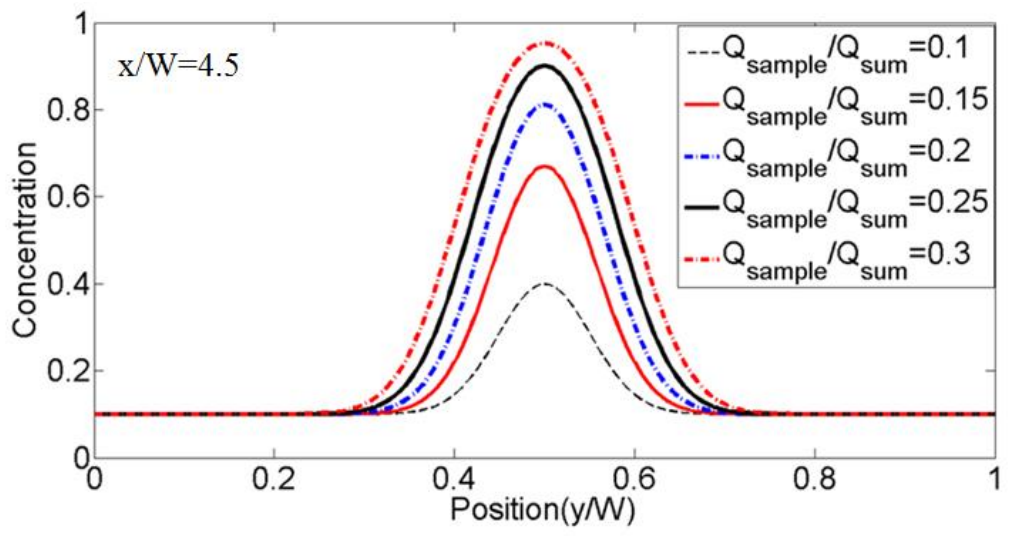

Figure 0-7 Concentration profiles for varied relative flow rate.

When the two sheath flow rates are equal, the concentration peaks in the different cross section are on the channel center line. When the sheath flow rates are different, the peak concentration position will depart from the center line. Hence adjusting the sheath flow rate can adjust the concentration peak position. This phenomenon can be used to obtain a better signal in a Coulter counter device. Under certain assumptions the peak concentration location can be calculated theoretically. First, we assume the flow is fully developed away from the inlet, so the velocity distribution profile has the analytic form $\left[u(y) / u_{\text {mean }}\right]=6(y / W)-6(y / W)^{2}$ (here $\mathrm{y}$ is the vertical coordinate value of the sampled profile point). Second, we assume that the three streams have the same viscosity and they are immiscible. For immiscible streams, flow injected from each inlet will remain an identifiable region in the fully developed region. So the width of each region can be obtained through mass conservation.

$$
v_{1} \times 1=\int_{p}^{1}\left[6(y / W)-6(y / W)^{2}\right] d y
$$




$$
v_{3} \times 1=\int_{0}^{q}\left[6(y / W)-6(y / W)^{2}\right] d y
$$

$v_{1}$ and $v_{3}$ are the velocity of the top and bottom sheath stream, respectively, $H_{t o p}$ and $H_{\text {bottom }}$ are the y-coordinates marking the sample and sheath stream boundaries (Figure 0-8). Integrating Eq.(IV.1) and Eq.(IV.2), we get the coordinate value of $H_{\text {top }}$ and $H_{\text {bottom }}$. Then the location of the center of the sample stream is estimated to be

$$
e=\frac{\left|H_{t o p}+H_{b o t t o m}-1\right|}{2}
$$

Note that Eq.(IV.3) assumes that the distribution of the ion concentration at each cross section is symmetric about the peak concentration axis, which only happens when top and bottom sheath stream flow rates are the same. Considering the value of Peclet number is very high (2000), we consider Eq.(IV.3) is valid when the difference between $v_{1}$ and $v_{3}$ is small.

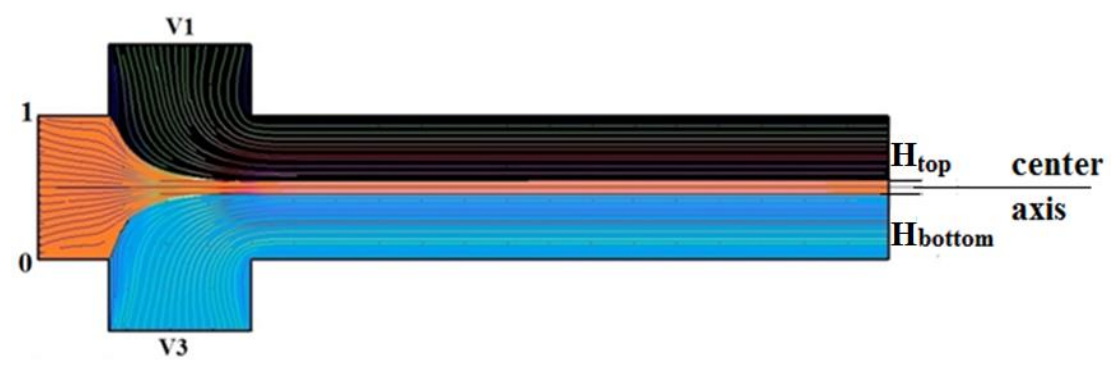

Figure 0-8 The micro channel with the mark of interface.

In order to confirm our analytical formula, a numerical simulation is tested. Four cases are simulated including the symmetric situation. The sample stream flow rate is fixed at 
$Q_{\text {sample }} / Q_{\text {sum }}=0.2$ while the difference between the rates of the two sheath streams $\Delta\left(Q_{\text {sheath }} / Q_{\text {sum }}\right)$ are varies from 0.05 to 0.15 . Figure $0-9$ shows the concentration profiles at $x=4.5$ by numerical simulation. The position of the peak concentration moves away from the center line as the difference between top and bottom sheath stream flow rates increases. Table 0-1 shows the distance of the peak position from the centerline at $x / W=2$ when the two sheath flow rates are different. As the difference between the two inlet flow rates increases the peak concentration moves further away from the center line. Comparisons are also made between the analytical (Eq.(IV.3)) and numerical results. Here we assume that the fluid rate from the bottom inlet is larger than the rates from the top inlet.

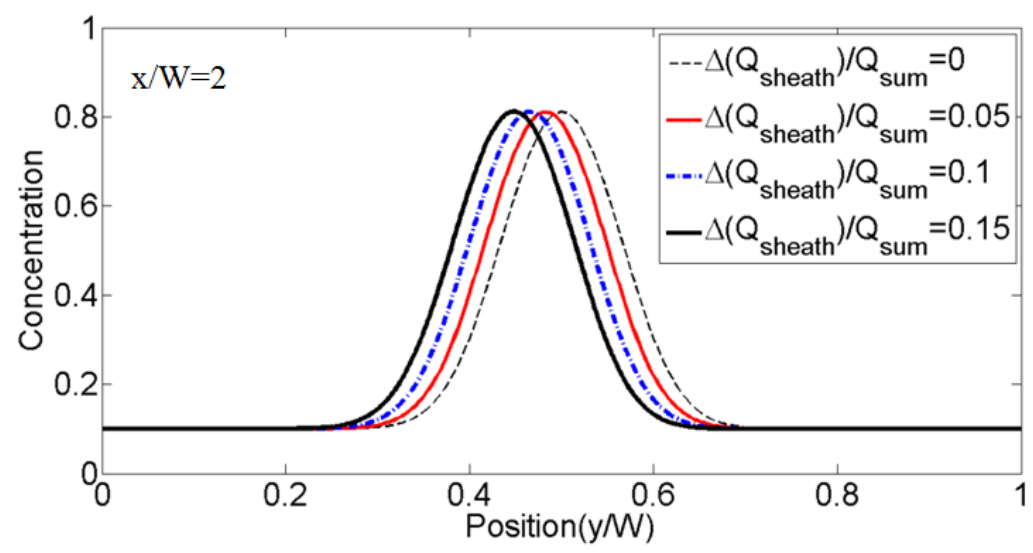

Figure 0-9 Concentration distribution with different sheath flow rates differences.

\begin{tabular}{|c|c|c|c|}
\hline & $\Delta\left(Q_{\text {sheath }} / Q_{\text {sum }}\right)=0.05$ & $\Delta\left(Q_{\text {sheath }} / Q_{\text {sum }}\right)=0.1$ & $\Delta\left(Q_{\text {sheath }} / Q_{\text {sum }}\right)=0.15$ \\
\hline $\begin{array}{c}\text { Numerical } \\
\text { solution }\end{array}$ & 0.016 & 0.033 & 0.053 \\
\hline $\begin{array}{c}\text { Analytical } \\
\text { solution }\end{array}$ & 0.017 & 0.033 & 0.051 \\
\hline
\end{tabular}




\begin{tabular}{|c|c|c|c|}
\hline $\begin{array}{c}\text { Relative } \\
\text { error }\end{array}$ & $5.8 \%$ & $0 \%$ & $3.9 \%$ \\
\hline
\end{tabular}

Table 0-1 Comparison of displacement from analytical and numerical simulations.

4.5 Three dimensional simulation for the hydrodynamic focusing

The micro channel Coulter counter fabricated in the clean room is three dimensional. So a three dimensional simulation will be better and more useful for scientists to investigate the hydrodynamic focusing phenomenon. Here a three dimensional simulation is processed to analyze the ion concentration distribution. This analysis is based on the geometry like Figure III-5. The size of the micro channel is: length $L=6.5$, width of the each inlet $W=1$ and the height of the micro channel $H=0.6$. Our sample stream flow rates are defined as $Q_{\text {sample }} / Q_{\text {sum }}=0.2$. The Reynolds number is 1 and the Peclet number is 2000 . The concentration of the sample stream at the inlet is 1 while the concentration of the sheath stream at the inlet is 0.1 . Figure $0-10$ shows the ion concentration contour for the hydrodynamic focusing. The result shows clearly that the sample stream is focused by the two sheath streams and becomes a narrow layer which is clamped by the two sheath streams at the downstream location. 


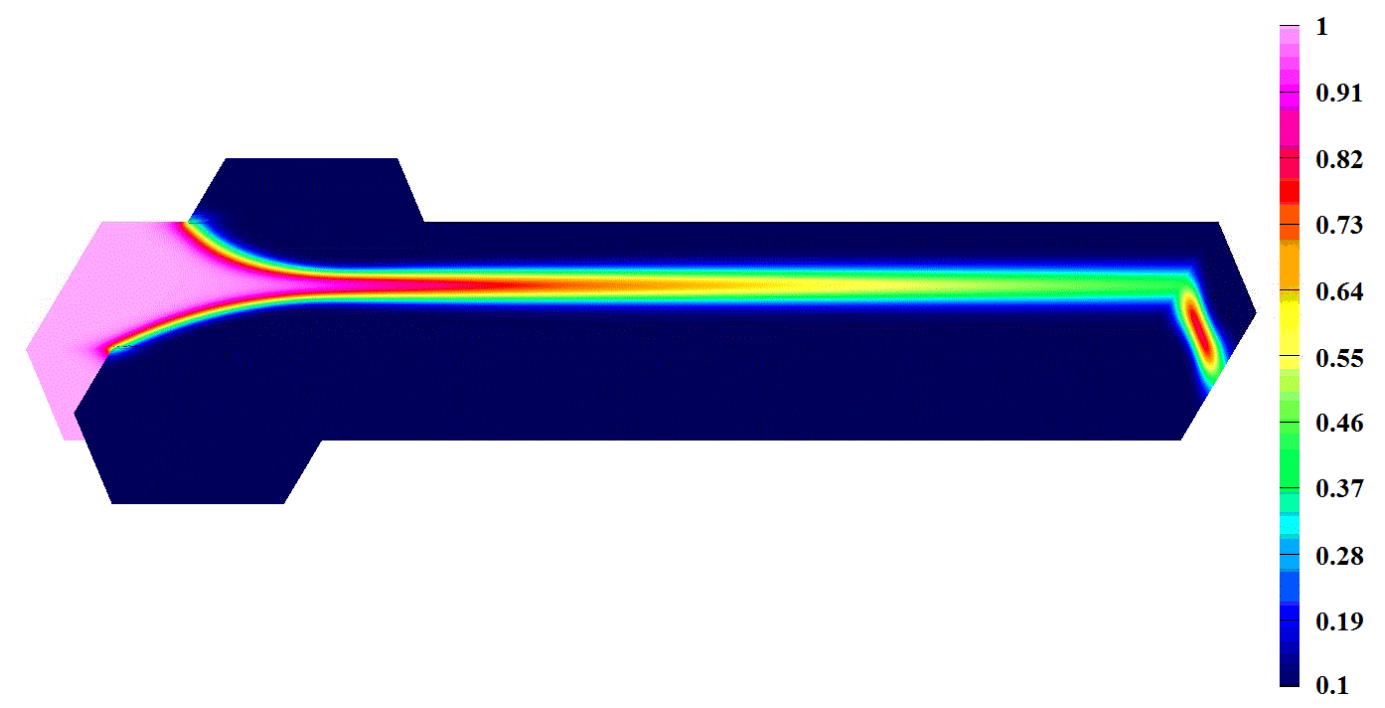

Figure 0-10 Ion concentration distribution for hydrodynamic focusing.

Figure 0-11 shows the concentration distribution profiles which are at four different positions: $x / W=3,4,5,6$. We can see that due to the impact of the diffusion phenomenon, when the position of the cross section moves towards the downstream, the ion concentration distribution becomes more flat. What is more, Figure 0-11 shows the vector distribution for the velocity in $\mathrm{x}$ and $\mathrm{y}$ direction in the plane of $x / W=3$. It shows clearly that there are four vortexes (second flow) in this plane. The second flow causes shear-augmented dispersion of ions. That makes the diffusion of ion more rapid and drag the shape of the sample stream like a dumbbell. 


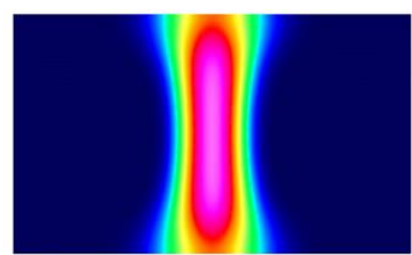

$\mathbf{a}(\mathrm{x} / \mathrm{W}=\mathbf{3})$

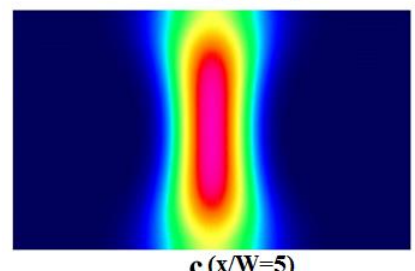

$\mathbf{c}(\mathrm{x} / \mathrm{W}=\mathbf{5})$

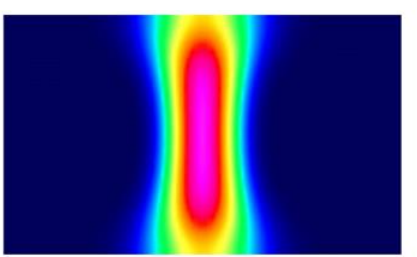

$b(x / W=4)$

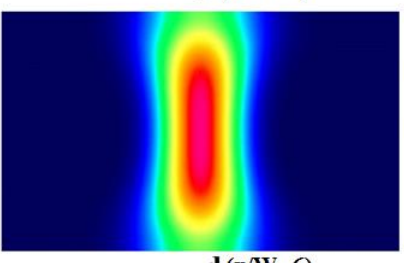

$\mathbf{d}(\mathrm{x} / \mathrm{W}=\mathbf{6})$

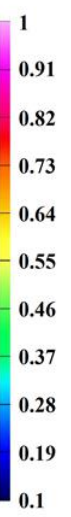

.82

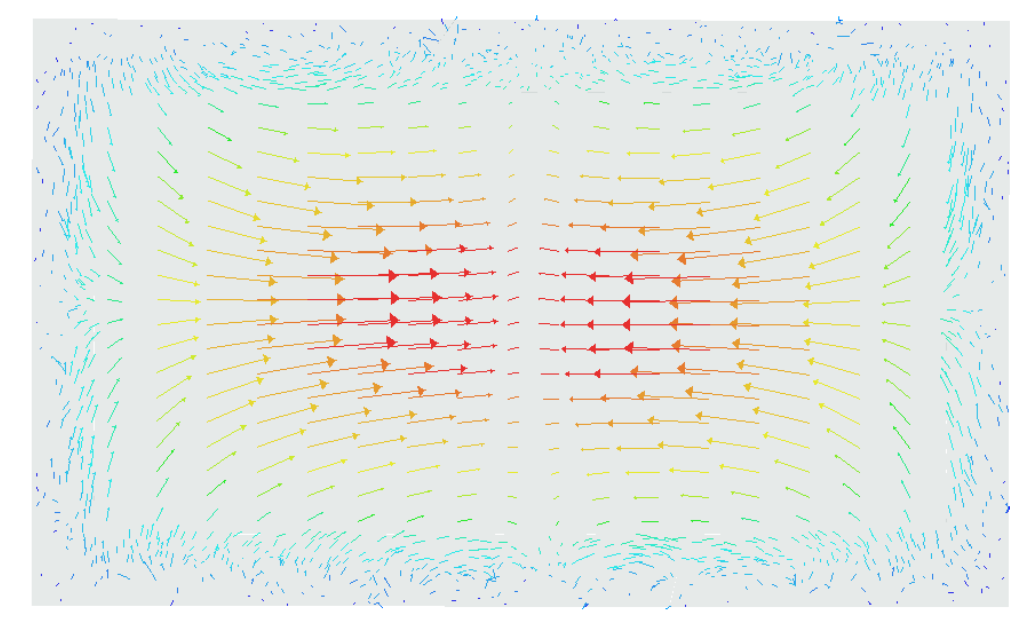

Figure 0-11 Contours of ion concentration distribution at different downstream positions and the fluid vector on the cross section of $x / W=3$. 


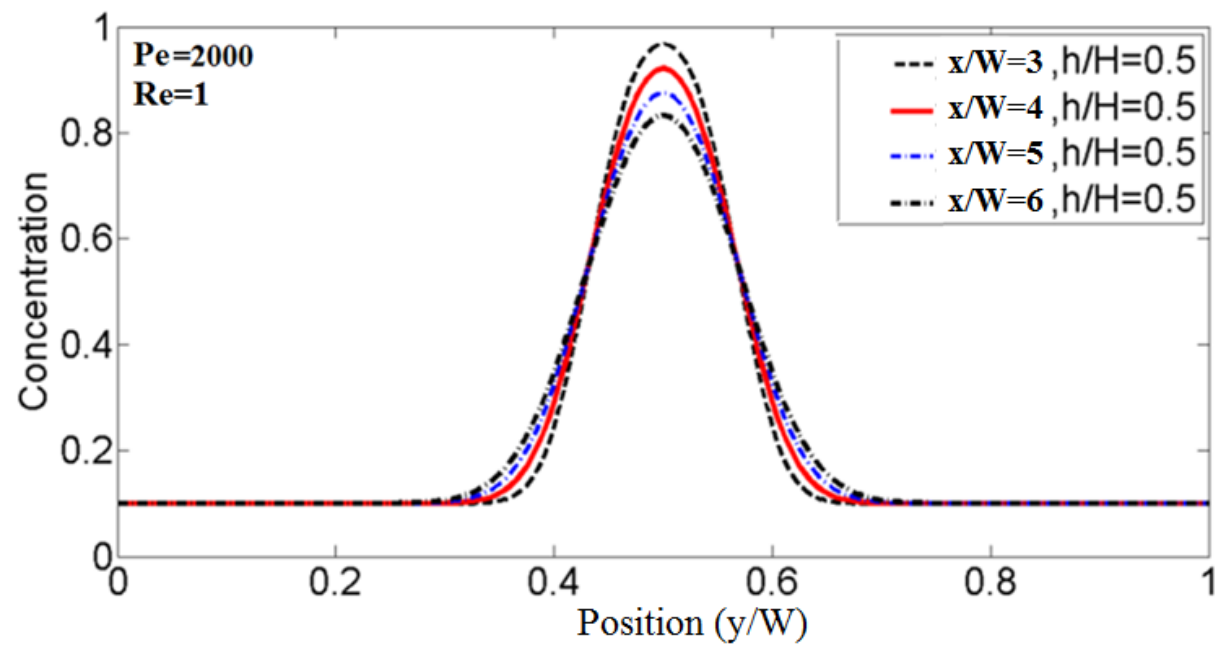

Figure 0-12 Lines of the ion concentration distribution at different positions.

Figure 0-12 shows the concentration distribution at four different lines on the middle plate. The results also show that the ion distribution gets smoother with downstream distance.

Another perspective which we can follow to investigate is plotting the ion concentration contour at different heights of the micro channel. Figure $0-13$ shows this result. Here $z$ / $H$ means the ratio between the heights of the plates which we extract data to the total height of the micro channel. We can see that as the position of the exacted plate moves far away to the middle plate, the concentration diffusion becomes smoother. This is because of the fluid velocity distribution. the middle plate gets the larger velocity distribution, so the ions have less time to diffuse; while at the edge of the micro channel the velocity is smaller, so the diffusion phenomenon takes the dominate position. 


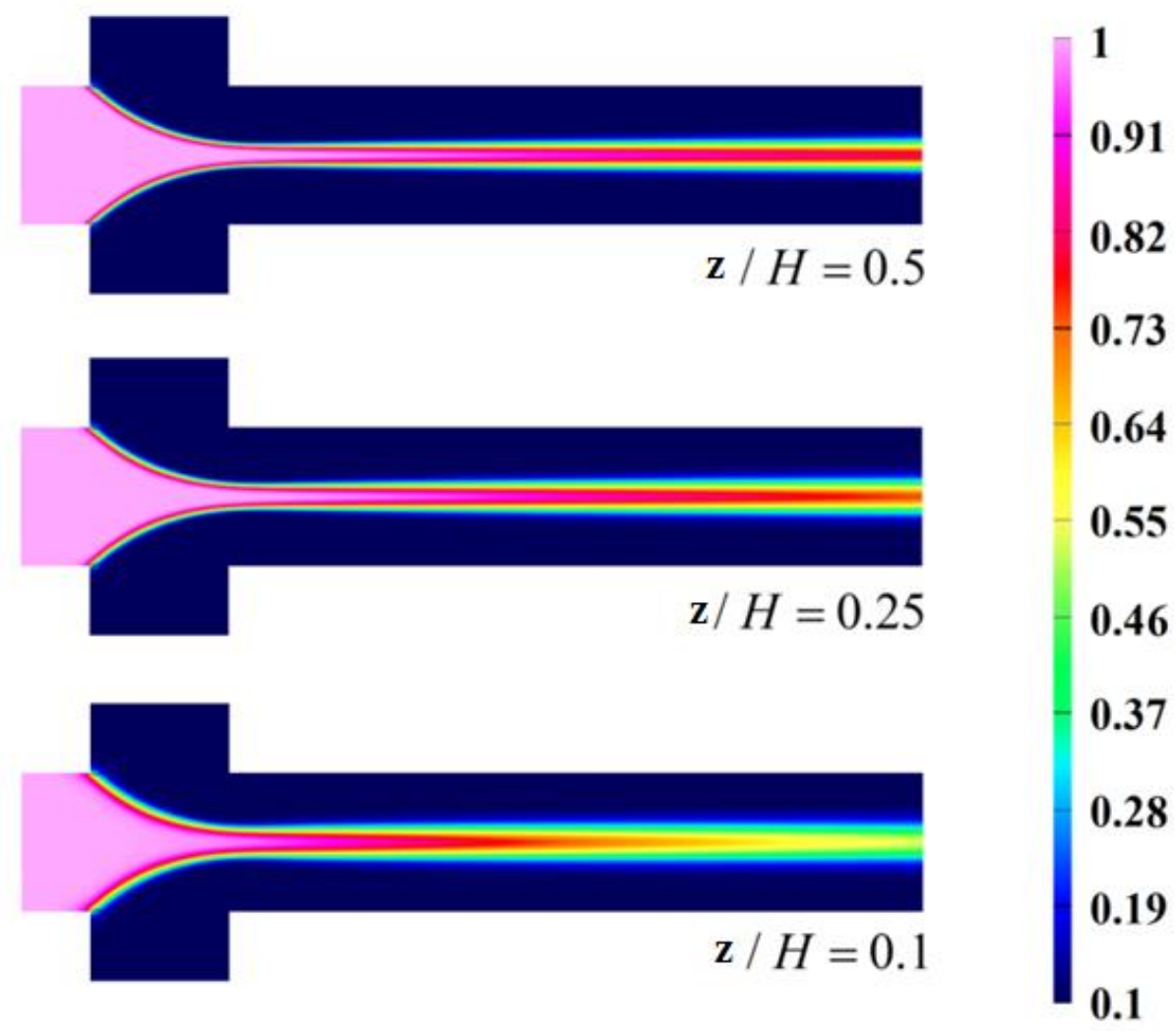

Figure 0-13 Cross sections of ion concentration distribution at the different $Z$ positions.

Figure 0-14 compares the concentration distribution along the center line for the variation of Reynolds number by fixing the Peclet number. In all three cases the sample flow rate is $Q_{\text {sample }} / Q_{\text {sum }}=0.2$. The Peclet number is fixed at 2000 . We can see that there is just a slight variation of the ion concentration distribution (the max relative error at the end of the vertex is no more than 2\%). Also Figure 0-14 compares the concentration profiles at $\mathrm{x} / \mathrm{W}=4.5$. It shows the same results that the variation of the Reynolds number from 1 to 10 has nearly no influence on the ion concentration distribution (the maximum relative error at the peak is no more than $2.4 \%$ ). 

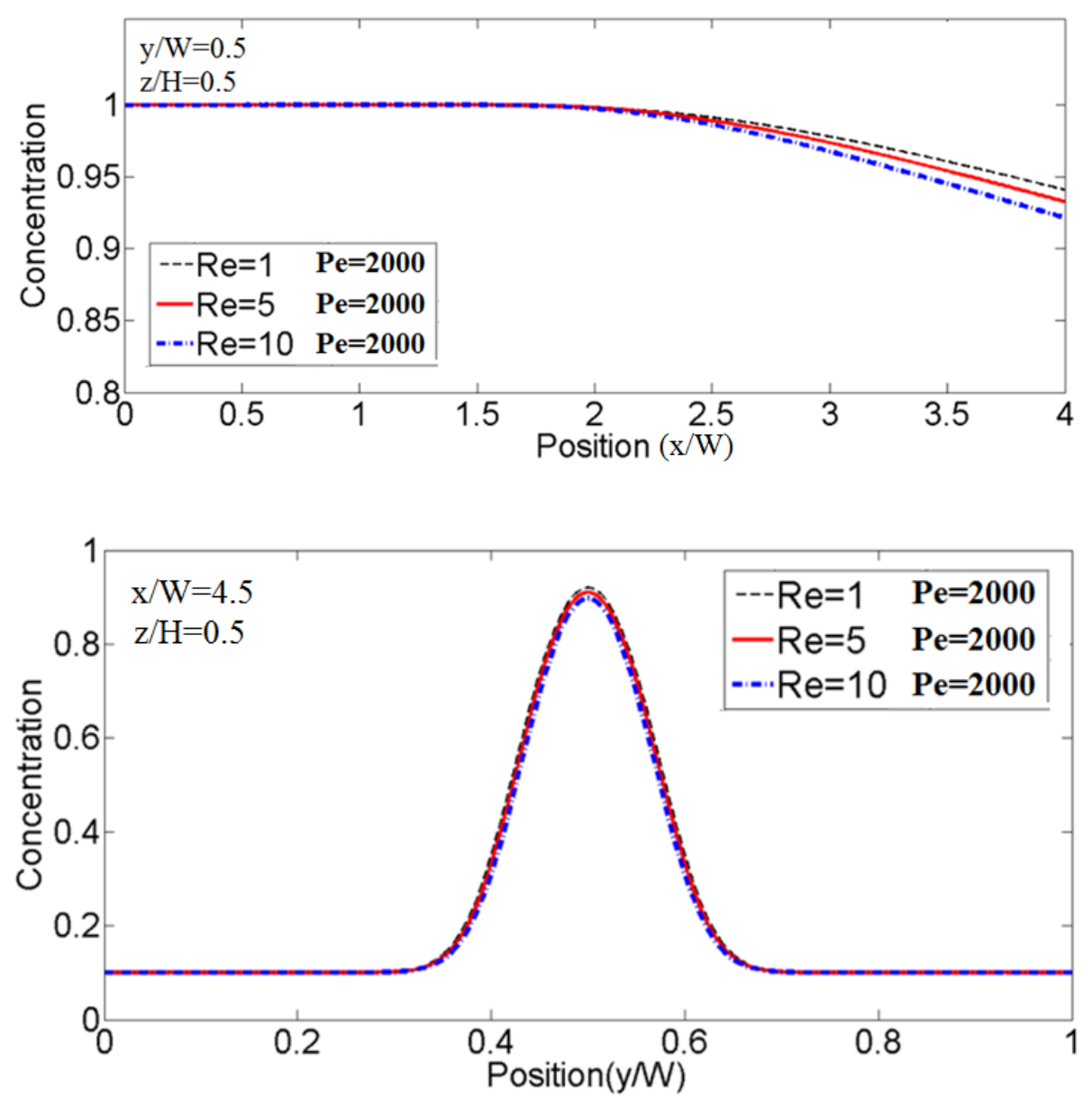

Figure 0-14 The concentration profiles at different Reynolds numbers by fixing the Peclet number.

Figure 0-15 compares the concentration distribution along the center line for the cases of different Reynolds number by fixing the Schmidt number. In all the three cases the sample flow rate is $Q_{\text {sample }} / Q_{\text {sum }}=0.2$. The Schmidt number is fixed on 1000. Also Figure 0-15 compares the concentration profiles at $\mathrm{x} / \mathrm{W}=4.5$ for the three cases. Both of them show the similar conclusion from 2D simulation that smaller Reynolds number can get smoother concentration distribution. This is also because under the condition of same 
Schmidt number, smaller Reynolds number can get larger diffusion coefficient, and that can make the concentration distribution smoother.
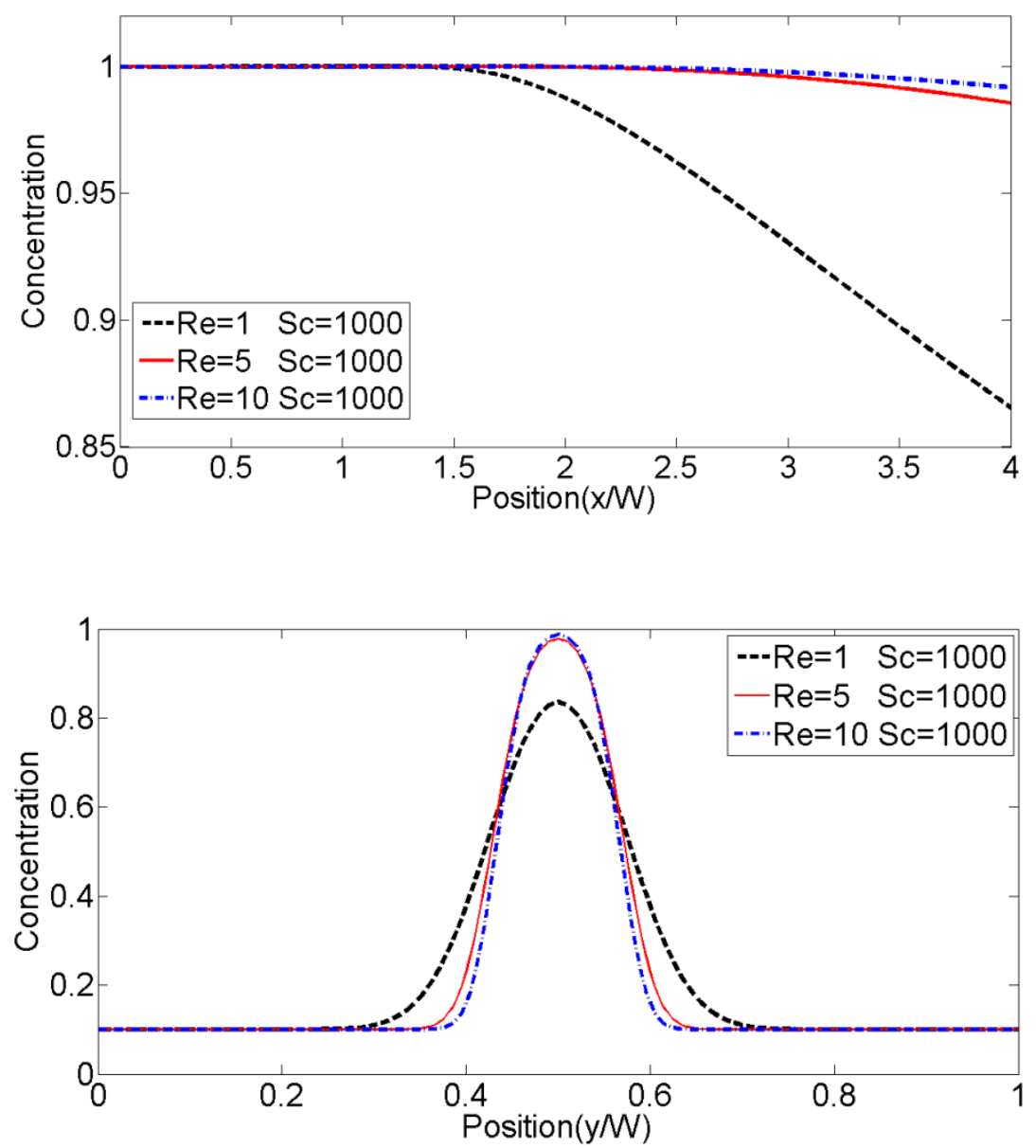

Figure 0-15 The concentration profiles at different Reynolds numbers by fixing the Schmidt number.

The impact of the Peclet number can be seen from Figure $0-16$. We extract the ion concentration distribution from the center line of the micro channel and it shows clearly that the larger Peclet number leads to the smaller molecular diffusivity coefficient and that will increase the ion concentration distribution. 


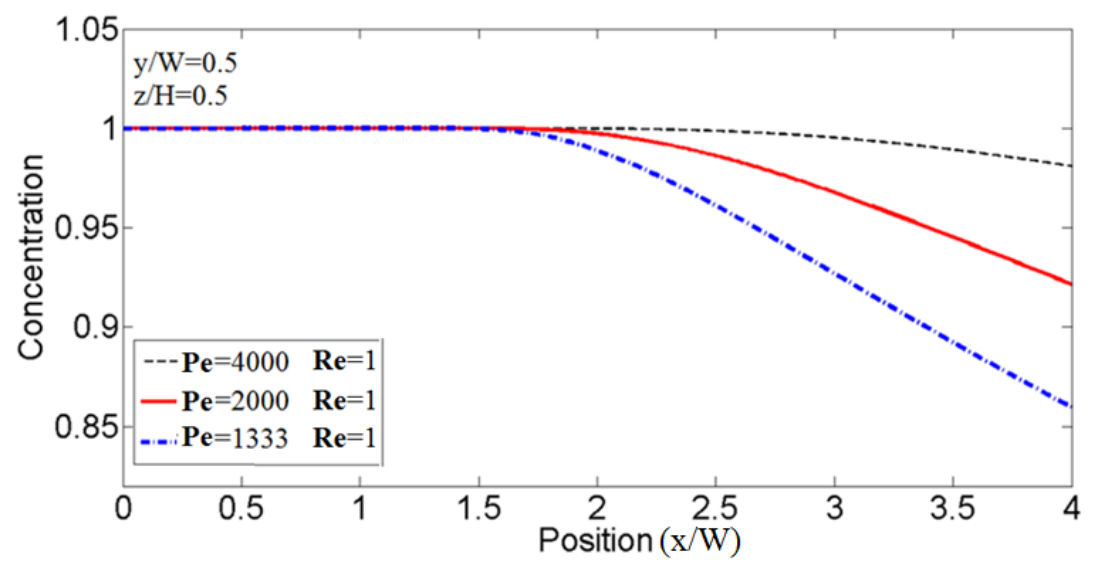

Figure 0-16 Concentration along the center line for varied Peclet number.

Also we can extract the data from a line which is on the middle plate and the line is parallel with y direction $(x / W=4.5, z / H=0.5)$. The results which are shown on Figure 0-17 lead to the same conclusion that the larger molecular diffusivity coefficient can cause the smoother ion concentration diffusion.

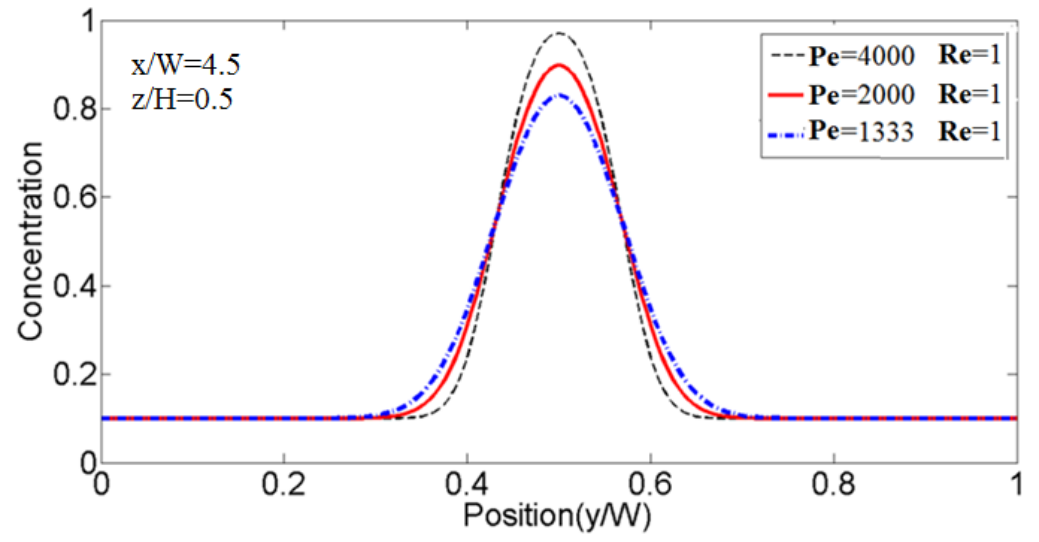

Figure 0-17 Impacts of the Peclet number variation.

Figure 0-18 shows the impacts of the sample stream flow rates variation. The sample stream flow rate is set as $Q_{\text {sample }} / Q_{\text {sum }}=0.1,0.15,0.2,0.25,0.3$ while the sum of the three inlets rates is fixed to one. Here we can see the results which are extracted from the 
center axis. It shows clearly that the larger sample stream flow rate can lead to larger ion concentration distribution.

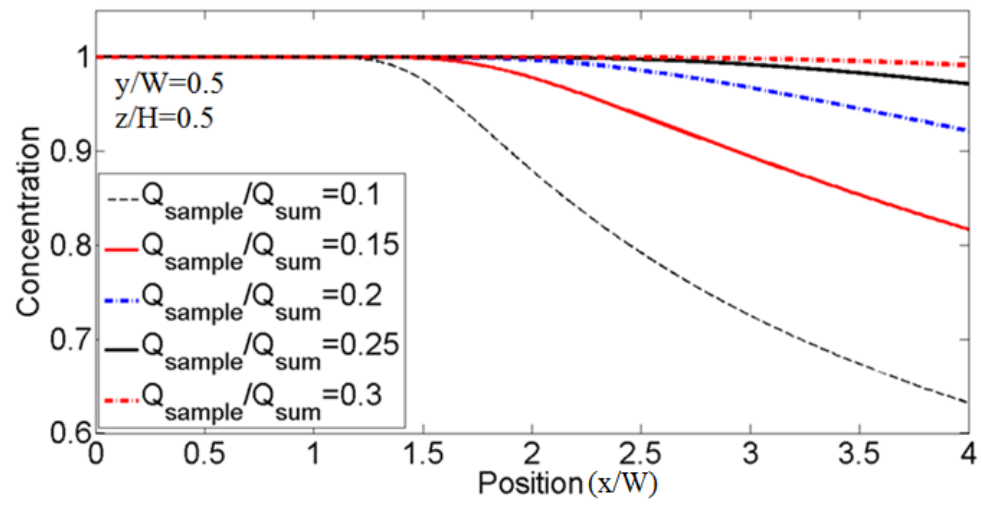

Figure 0-18 Concentration along the center line for varied relative flow rates.

A similar tendency is shown in Figure 0-19. Here we choose the centerline as before. The results also show the same conclusions as we talked before: The larger sample stream flow rate can lead to larger ion concentration distribution.

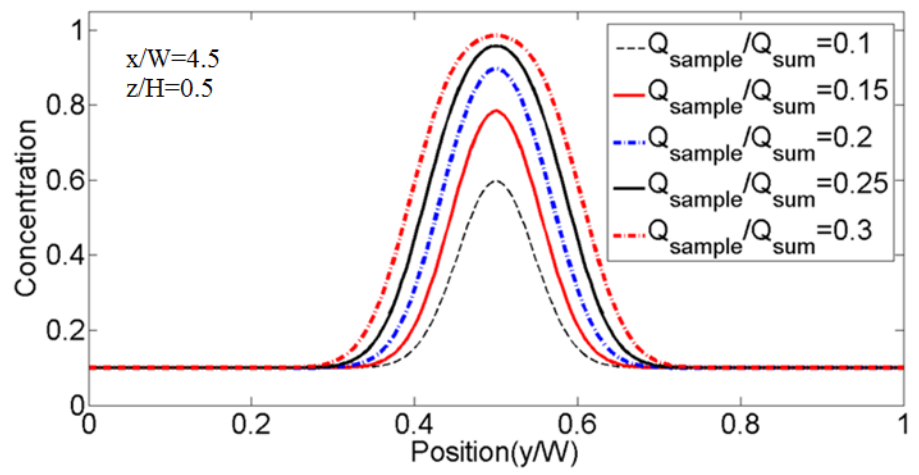

Figure 0-19 Concentration along the fixed line for varied relative flow rates.

Then we can have a look about the asymmetric situation under the two dimensional simulation by three dimensional simulation. Here $Q_{\text {sample }} / Q_{\text {sum }}=0.2$ and we also prefer to 
do three different simulation cases with the fluid rate differences of the two sheath stream: $\Delta\left(Q_{\text {sheath }}\right) / Q_{\text {sum }}=0.05, \Delta\left(Q_{\text {sheath }}\right) / Q_{\text {sum }}=0.1$ and $\Delta\left(Q_{\text {sheath }}\right) / Q_{\text {sum }}=0.15$. Figure $0-20$ Shows the ion concentration distribution at the same cross section position under the three different conditions. It shows that the focused sample stream is pushed towards left due the difference of the sheath stream flow rates.

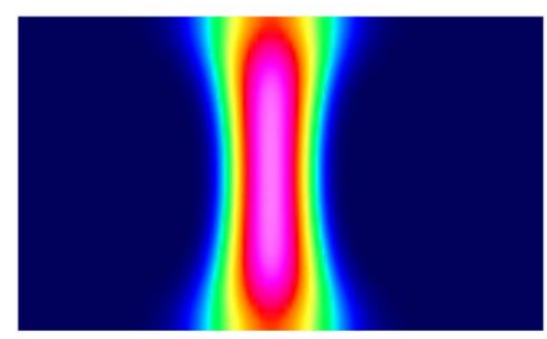

$\Delta\left(Q_{\text {sheath }}\right) / Q_{\text {sum }}=0.05$

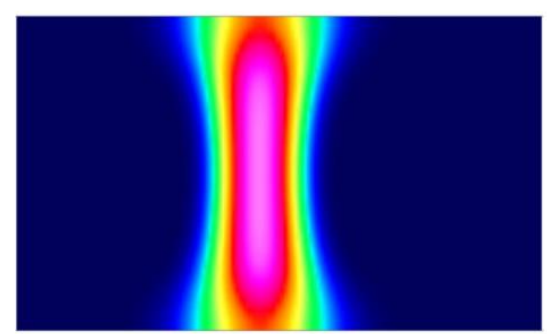

$\Delta\left(Q_{\text {sheath }}\right) / Q_{\text {sum }}=0.1$

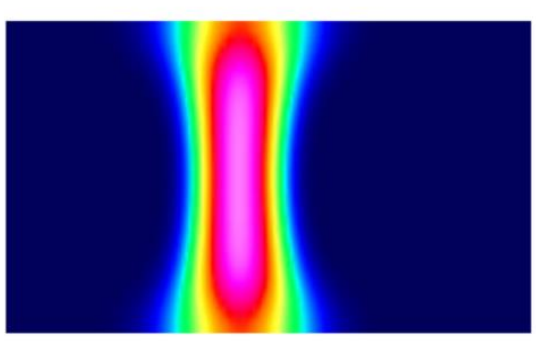

$\Delta\left(Q_{\text {sheath }}\right) / Q_{\text {sum }}=0.15$

Figure 0-20 ion concentration distribution under the different asymmetric situation.

Then we have a study about the correlation between the concentration peak biases to the sheath stream flow rates difference. As the Figure 0-21 shows, we choose the gap from 
the max concentration peak at the middle plane to the geometric center as the concentration peak bias. Here we choose five different cases of sheath stream flow rates difference which are tested $\left(\Delta\left(Q_{\text {sheath }}\right) / Q_{\text {sum }}=0,0.5,0.1,0.15,0.2\right)$ while we fix the total fluid rate and the sample stream flow rate is fixed at $Q_{\text {sample }} / Q_{\text {sum }}=0.2$. The results at $x / L=0.2$ are shown on Figure $0-22$. We can see that the bias increases when the sheath stream flow rates difference increases. Also the solution shows that there is an approximate linear relation between these two parameters.

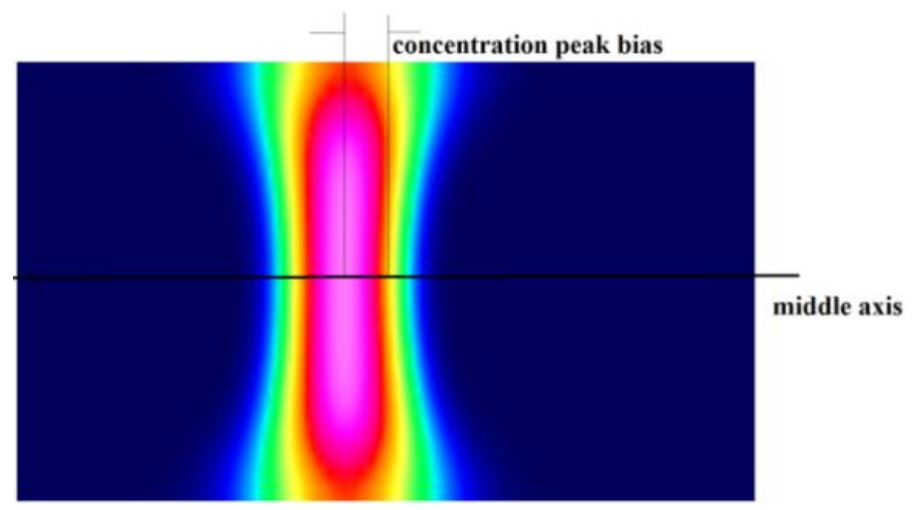

Figure 0-21 Definition of the concentration peak bias under the asymmetry phenomenon.

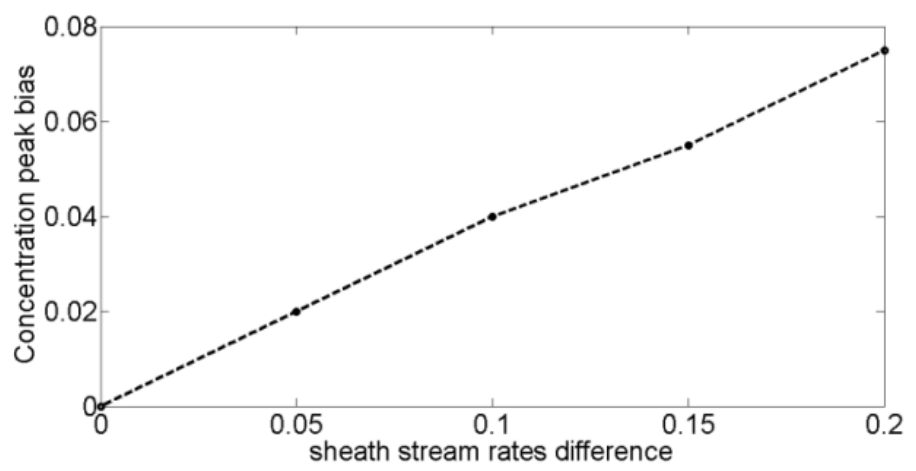

Figure 0-22 Correlation between the concentration peak bias and sheath stream flow rates difference. 
Another aspect about the correlation between the concentration peak bias and the sample stream flow rates is investigated. We do not change the total fluid rate and fix the sheath stream flow rates difference as $\Delta\left(Q_{\text {sheath }} / Q_{\text {sum }}\right)=0.2$. Three different cases are simulated $\left(Q_{\text {sample }} / Q_{\text {sum }}=0.15,0.20 .25\right)$. The solution from Figure $0-23$ shows the concentration peak bias does not change. It means the sample stream flow rate does not affect the concentration peak bias.

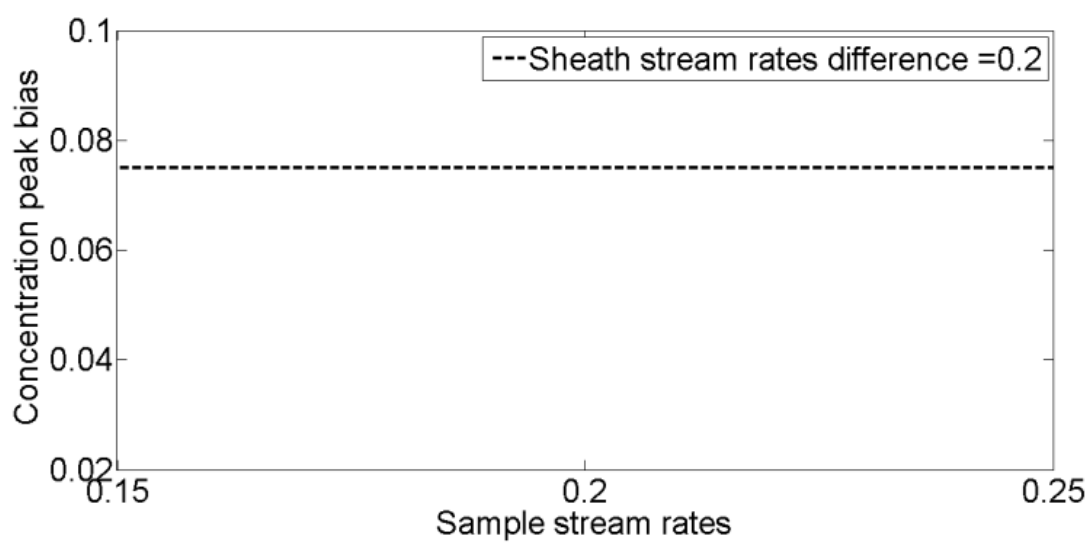

\section{Figure 0-23 Correlation between the concentration peak bias and sample stream flow rates.}

Then the impact of the micro channel height is studied. Three different cases are simulated (height $=0.6,0.8,1$ ). The sample stream flow rate is fixed at $Q_{\text {sample }} / Q_{\text {sum }}=0.2$. The sheath stream flow rates difference is fixed at $\Delta\left(Q_{\text {sheath }}\right) / Q_{\text {sum }}=0.2$. The results from Figure 0-24 show that when the height of the micro channel is increased, the bias is also 


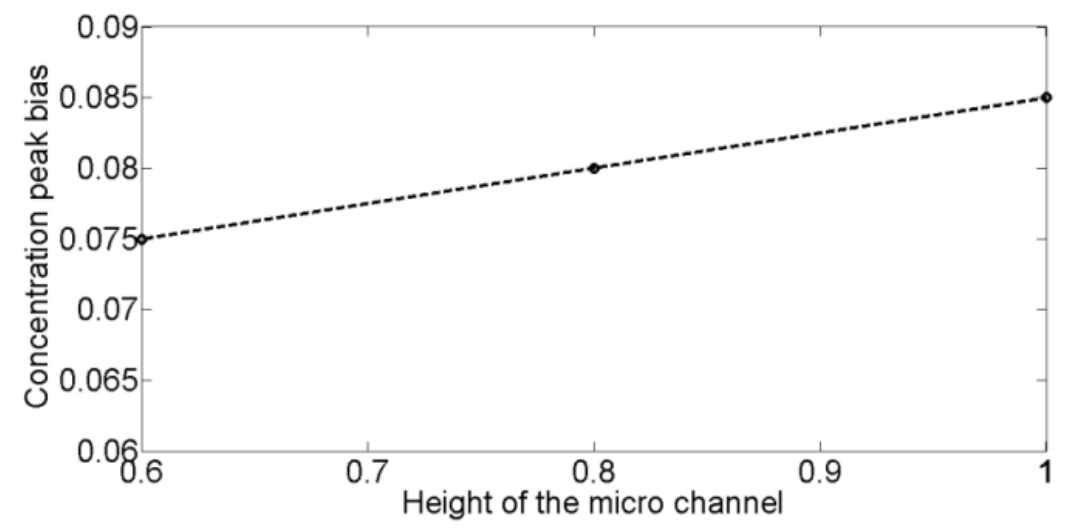

\section{Figure 0-24 The correlation between the concentration peak bias and micro channel height.}

increased. Also there is a linear relation between these two parameters.

\subsection{Particle motion study}

Hydrodynamic focusing can not only confine the sample stream but it can focus particles into a stream at desirable positions. To examine the focusing capabilities, the configuration with three micro channel inlets (see the configuration shown in Figure 0-25) was used. The two sheath streams had the same flow rate, $Q_{\text {sheath }} / Q_{\text {sum }}=0.4$ and $Q_{\text {sample }} / Q_{\text {sum }}=0.2$. A particle (radius 0.02 ) was initially placed above the center line. The bias distance from the center line was 0.2. Under the hydrodynamic forces, the particle moves toward the center line due to hydrodynamic focusing. Figure $0-25$ shows the snapshots of particle position at different time instants (the red circle represents the particle, in order the show its position clearly, its radius is much larger than 0.02). 

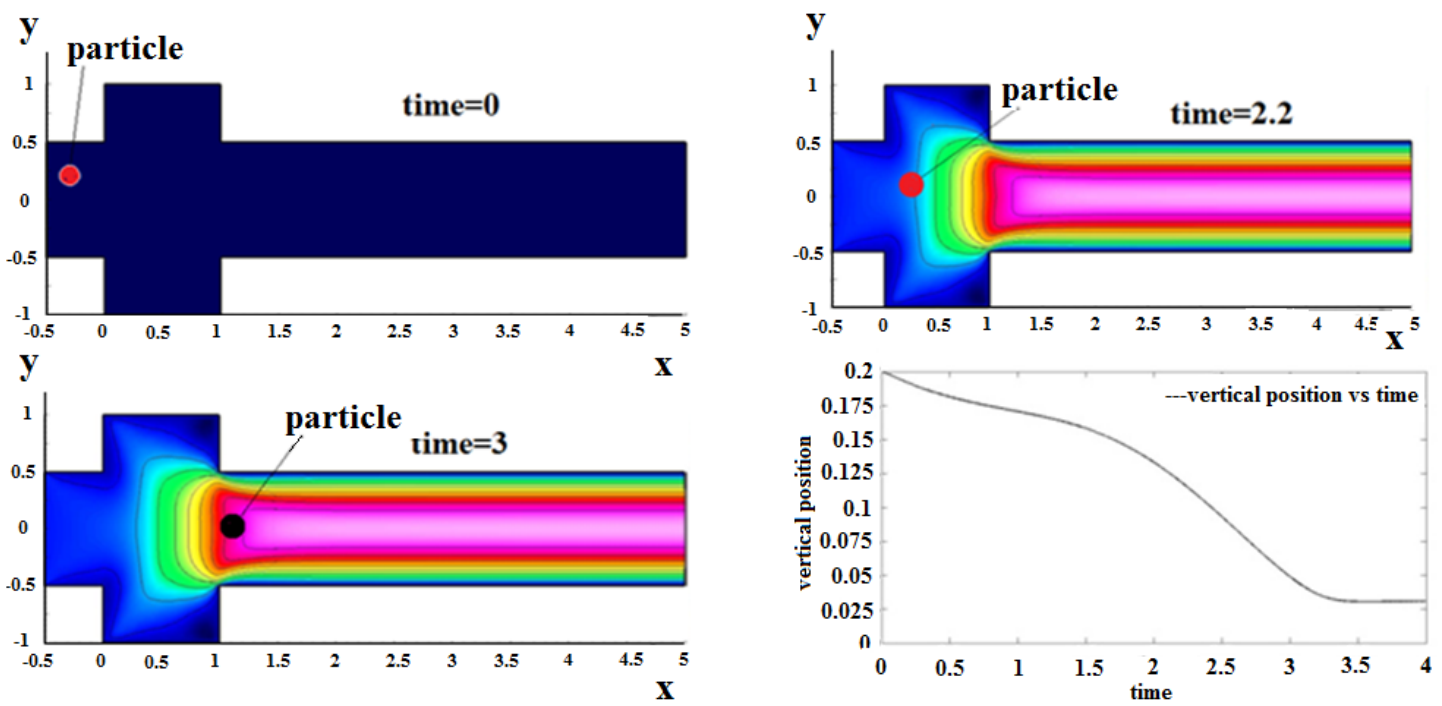

Figure 0-25 The motion of a particle under hydrodynamic focusing (single particle).

When too many particles pass the detection area they may jam the detecting devices or lead to poor signal. Hydrodynamic focusing can be used to separate initially close particles so a more distinct signal can be obtained when the particle pass through the electrical field. To demonstrate this we simulated two initially close particles moving in the hydrodynamic focused flow region. Figure 0-26 shows the computation grid and the initial positions of the two particles. Here the two sheath flow streams have the same flow rate. The original position of the two particles is on the center line and their initial separation distance is 0.2 .

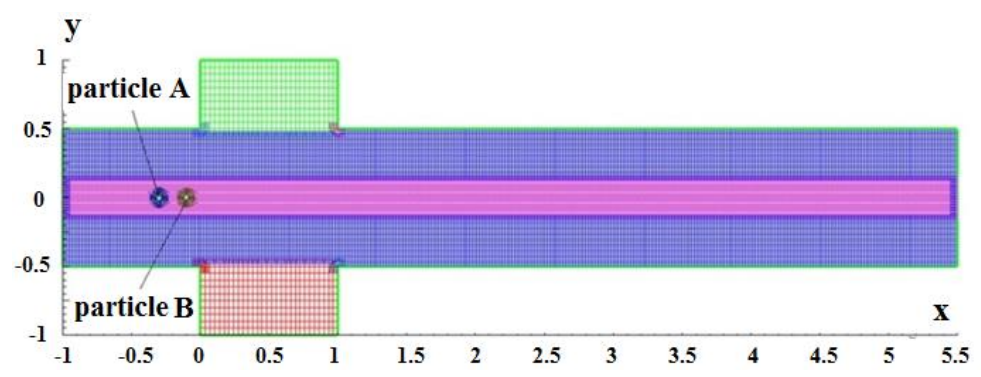

Figure 0-26 The grids for the simulation of two particles in a micro channel. 
Figure 0-27 shows the numerical simulation under the condition of $Q_{\text {sheath }} / Q_{\text {sum }}=0.45$ and $Q_{\text {sample }} / Q_{\text {sum }}=0.1$. As the two particles move downstream of the junction, their separation increases.
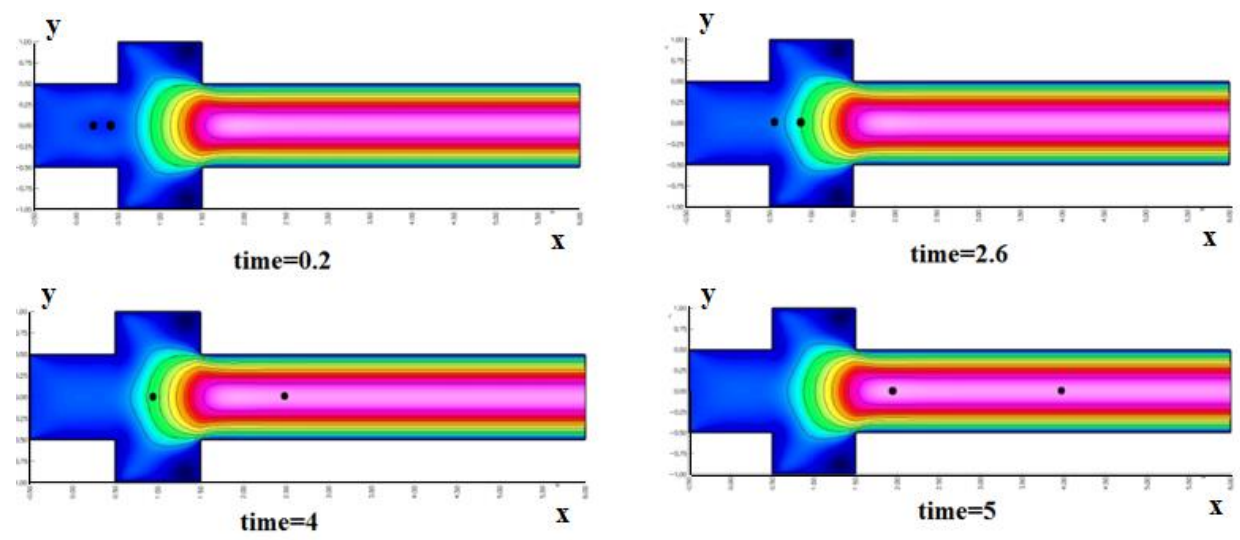

Figure 0-27 Snapshots of the particle motion in a micro-channel.

Figure $0-28$ shows distance between two particles at different time instants. Different sample stream flow rates are used while the initial particle gap is fixed at 0.2. We can see clearly that as $Q_{\text {sample }} / Q_{\text {sum }}$ increases the gap that particles between each other at final decreases. In practice, we can adjust $Q_{\text {sample }} / Q_{\text {sum }}$ to get the available final distance. Normally, the large final distance between the adjacent particles is better for the equipment to accurately count. 


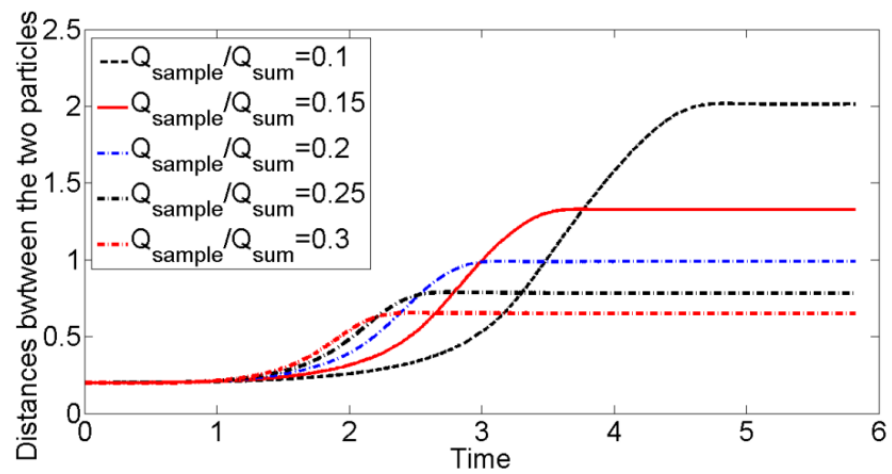

Figure 0-28 Distance between the two particles as a function of time.

The same conclusion is demonstrated for the case of multiple particles which is shown in

Figure 0-29. (Since there are multiple particles, the length of the center inlet is increased to 1$)$.
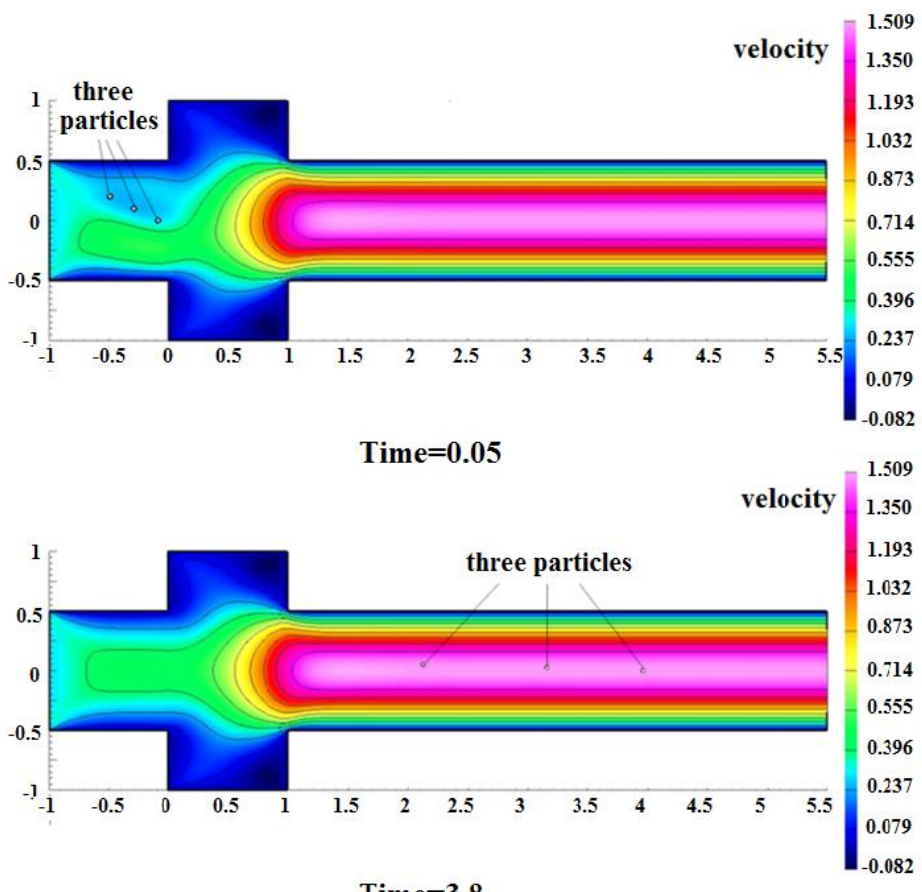

Figure 0-29 multiple particles moving in the micro channel. 
Similar as $2 \mathrm{D}$ simulation, the distances between two particles are investigated by $3 \mathrm{D}$ simulation, the contour of the distance variation is shown in Figure 0-30. It is done by fixing $Q_{\text {sample }} / Q_{\text {sum }}=0.3$ and the initial gap between the two particles is 0.3 . The solution also shows the hydrodynamic focusing can increase the horizontal distance of the two adjacent particles. More research is done by considering different sample stream flow rates. Figure 0-31 shows the results which fixes the initial gap between the two particles as 0.3 while changing the sample stream flow rate. The solution also gives us the same conclusion that decreasing the sample stream flow rates can increase the distance between two adjacent particles.

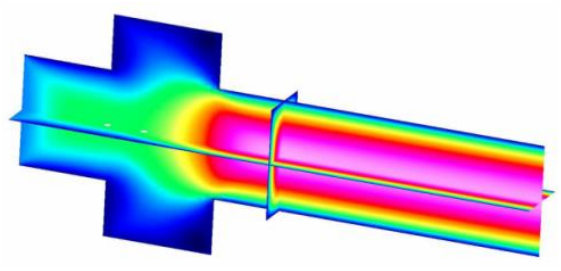

time $=\mathbf{0 . 9 8}$

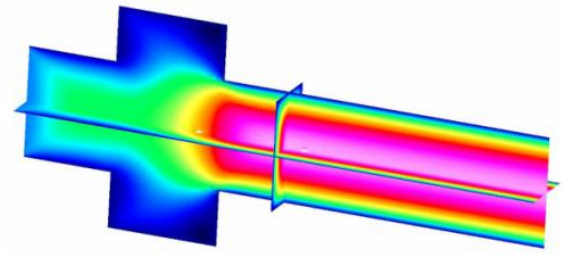

time $=\mathbf{2 . 0 4}$

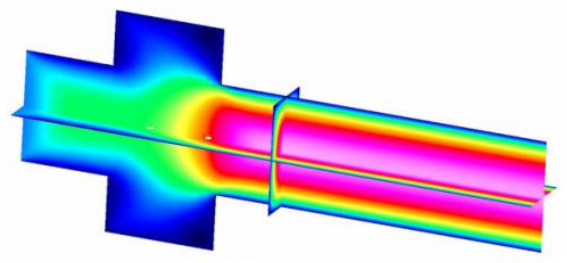

time $=1.58$

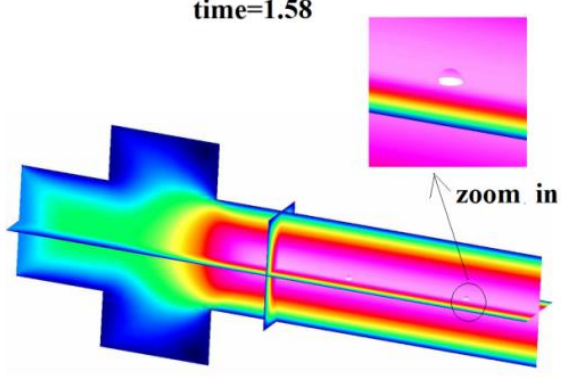

time $=\mathbf{2 . 8 2}$

Figure 0-30 Snapshots of the particle motion in a micro-channel by 3D simulation. 


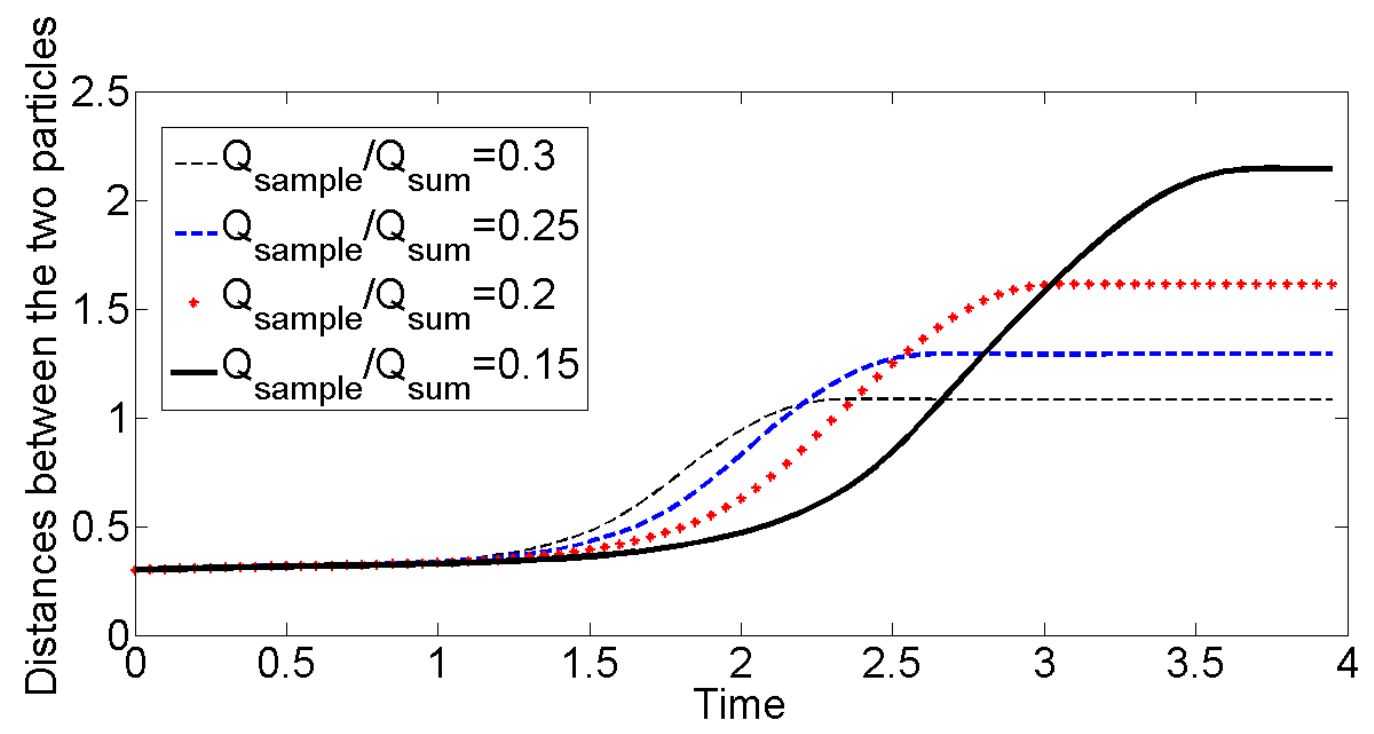

\section{Figure 0-31 Distance between the two particles as a function of time by 3D simulation.}

\subsection{Three-inlets/Three outlets micro channel study}

A relatively new Coulter counter configuration is shown in Figure 0-32. In this 2D configuration the device has three inlets and three outlets. In experiments the flow rate for each outlet can be controlled by adjusting the pressure at each outlet reservoir. With the three-inlet and three-outlet design, metal tubes deliver fluid to the center inlet and outlet while providing electrical contact to the conductive fluid at both ends of the focused stream. Normally, the electrical contacts are located at the entrance, where the stream is in the process of being focused; and exit, where the stream is focused and the result is a very small contact area. The new design, though has the risk of losing resolution, has the practical benefit of increasing contact area between the downstream electrode and the conductive solution in the central stream, thereby reducing electrical contact resistance when making impedance measurements, and increasing the sensitivity 
of the device to smaller particles that would otherwise be hidden by electrical noise. Our objective is investigating the stream concentration profiles

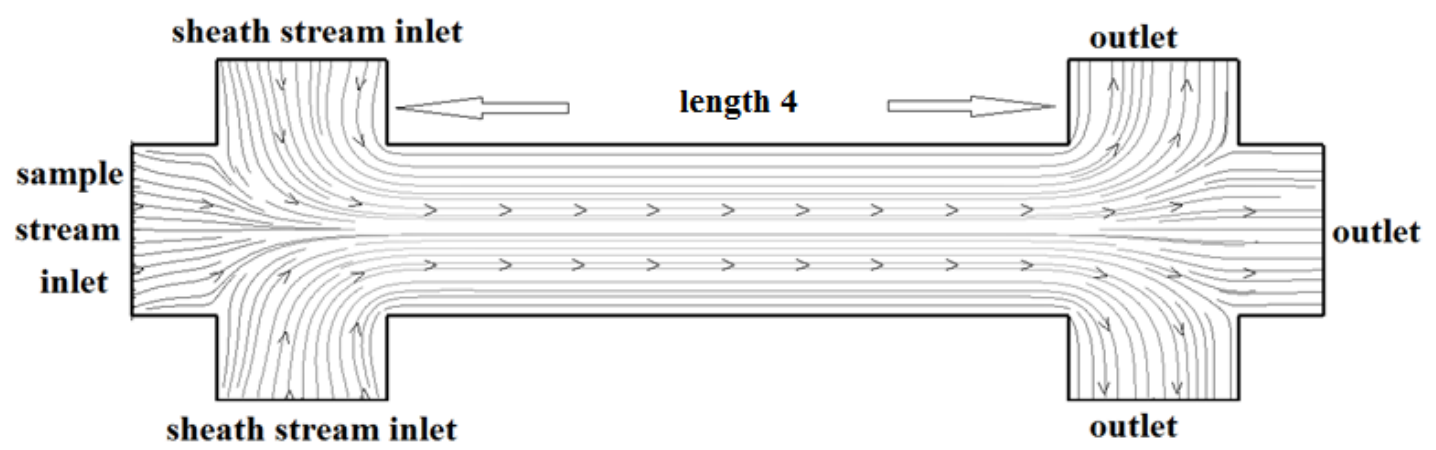

Figure 0-32 The streamlines in a three-inlet/three-outlet micro-channel device.

by adjusting the velocity of the sample stream and sheath stream while keeping their sum equal to one. Here we only consider the symmetrical case, which means the two sheath stream flow rates are the same. Figure 0-33 shows concentration contours with different relative flow rates. From these figures we can see that increasing the velocity of the

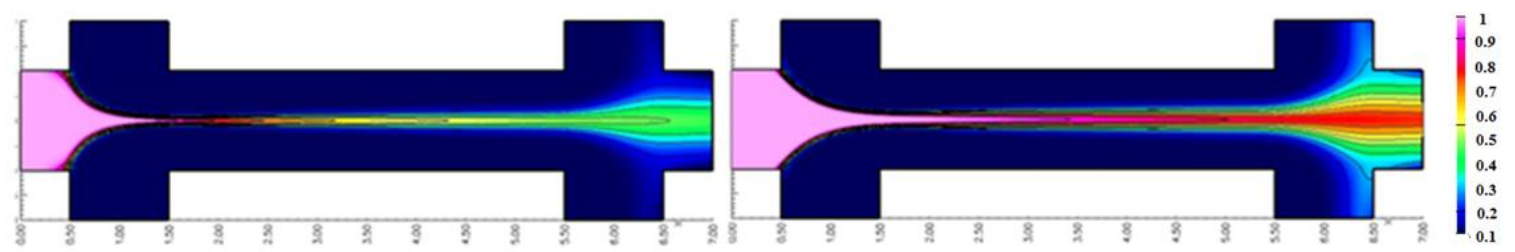

a

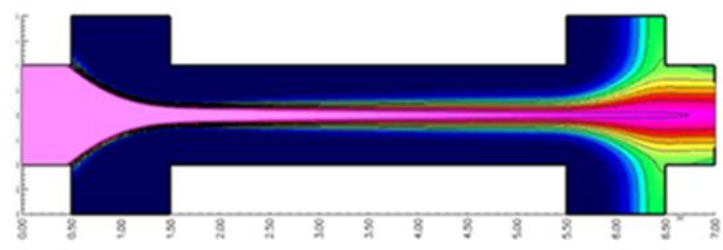

c b
a: $Q_{\text {sample }} / Q_{\text {sum }}=0.1$
b: $Q_{\text {sample }} / Q_{\text {sum }}=0.2$
c: $Q_{\text {sample }} / Q_{\text {sum }}=0.3$

Figure 0-33 Concentration contours with different sample stream flow rates. 
sample stream can increase the width of the diffusion region at the middle outlet. For a low value of $Q_{\text {sample }} / Q_{\text {sum }}=0.1$, nearly none of the conductive fluid of the sample stream moves to the sheath flow outlets. For $Q_{\text {sample }} / Q_{\text {sum }}=0.3$, it is clear that some of the sample stream fluid moves to the sheath flow outlets. 


\section{Summary}

This chapter gives a detailed numerical study about hydrodynamic focusing. The characteristics of the hydrodynamic focusing are discussed from several aspects including the dimensionless numbers, relative sheath stream/sample stream flow rates, asymmetric situation and so on. The simulation results show us that by fixing the Peclet number, the variation of the Reynolds number from one to ten has slight influence to the ion concentration distribution while smaller Reynolds number can get smoother concentration distribution if we fix the Schmidt number. Also increasing of the sample stream flow rate can increase the ion concentration value. These characteristics are confirmed both by two dimensional and three dimensional simulation. After that the impacts of the hydrodynamic focusing to the particle motion is discussed. The results confirm that the hydrodynamic focusing can separate particles in the horizontal direction while focus particles into the middle region in the vertical direction. Multiple cases are tested to show that decreasing the sample stream flow rate can enlarge the horizontal gap between particles. At last the chapter studies a three inlets/three outlets micro channel and the results show that the hydrodynamic focusing can create an ion concentration distribution with "dog-bone" shape. 


\section{TWO DIMENSIONAL SIMULATION FOR COULTER PRINCIPLE}

This chapter uses two dimensional simulation to study the current variation when the particle passes through the detection region. The simulation solver we choose to solve the electrical field has been validated before. Our numerical simulation will consider the impacts of the molecular diffusivity phenomenon and compare the results between the two cases which consider molecular diffusivity or not. Multiple physical/design parameters will be considered such as the particle size, electrodes gap, electrodes length, electrodes distribution and relative sheath/sample stream flow rate in order to investigate their influence to the current sensitivity. 
5.1 The impacts of molecular diffusion phenomenon

Figure V-1 shows hydrodynamic focusing happened under the condition where the Reynolds number is 1 . The sample rate is $Q_{\text {sample }} / Q_{\text {sum }}=0.3$. This figure shows that the ion concentration distribution in a three-inlet channel in which two low-conductive sheath flows are injected from the top and bottom inlets and the conductive sample flow is injected from the middle inlet. For the study of the electric field we focus on the marked region. The electrodes are fixed as the Figure II-2 shows, the width of the simulation domain is one (from $y=-0.5$ to $y=0.5$ ) while the length is 2 (from $x=6$ to $x=8$ ). The length of each electrode is 0.3 and the gap between the two electrodes is 0.6.

Figure V-2 compares the conductivity at $\mathrm{x} / \mathrm{W}=7$ with and without diffusivity. When diffusivity is considered, the conductivity distribution profile is more spread out. Because the electrical field is affected by the conductivity distribution, the diffusivity will also affect the current variation. Here we qualitatively analyze the influence of diffusivity on the current variation. Two cases are simulated and compared. In the first case no diffusivity is considered and in the second case $P e=1000$ is considered. In both cases we focus on a region marked within the rectangle in Figure V-1. The marked region has a length of two and a height of one. The electrodes are mounted at the bottom of the channel. The particle radius is 0.04 comparing with the width of the channel. The conductivity of the particle is 0.1 . At the inlet the conductivity of the sample stream and sheath streams is 1 and 0.1 , respectively. 


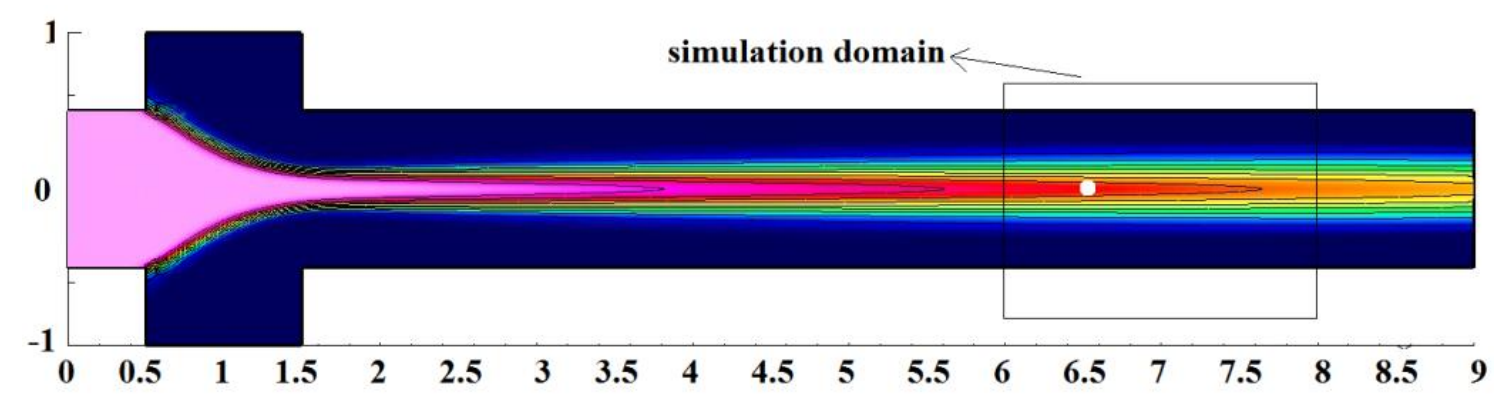

Figure V-1 Conductivity distribution in a micro fluid Coulter counter. Sheath flows are injected from top/bottom inlets and conductive flow is injected from middle inlet.

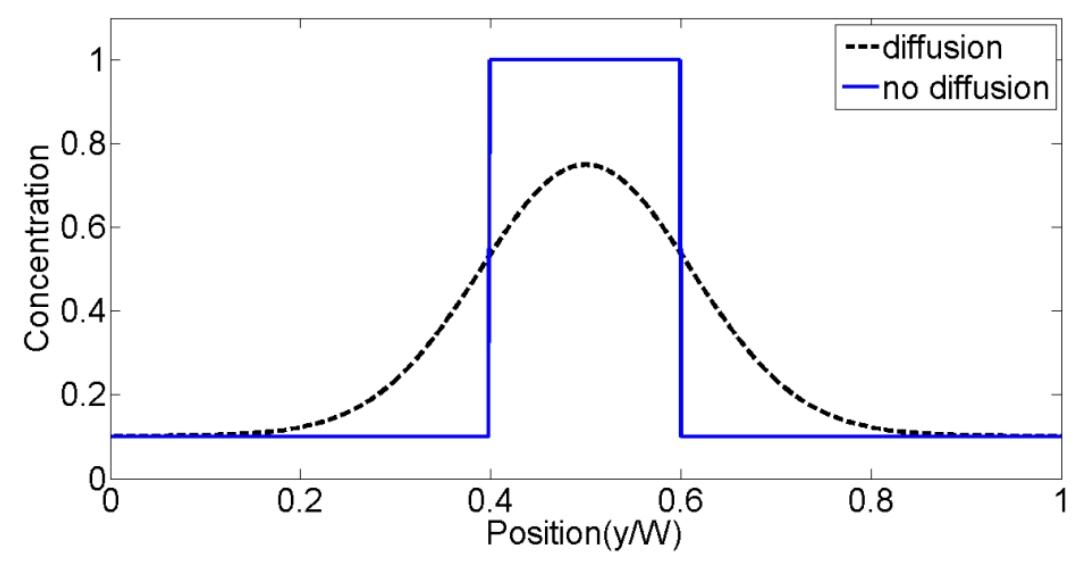

Figure V-2 Comparison of conductivity distribution of sample flows with and without molecular diffusivity at $\mathbf{x} / \mathrm{W}=7$.

The calculated current sensitivity with and without molecular diffusivity is shown in Figure V-3. Following the work of Spencer and Morgan (2011), we use $\Delta I / I$ to represent the current sensitivity. Here $I$ is the current when no particle exists, and $\Delta I$ is the current variation due to the particle. We can see that the current sensitivity is lower when diffusion is considered. 


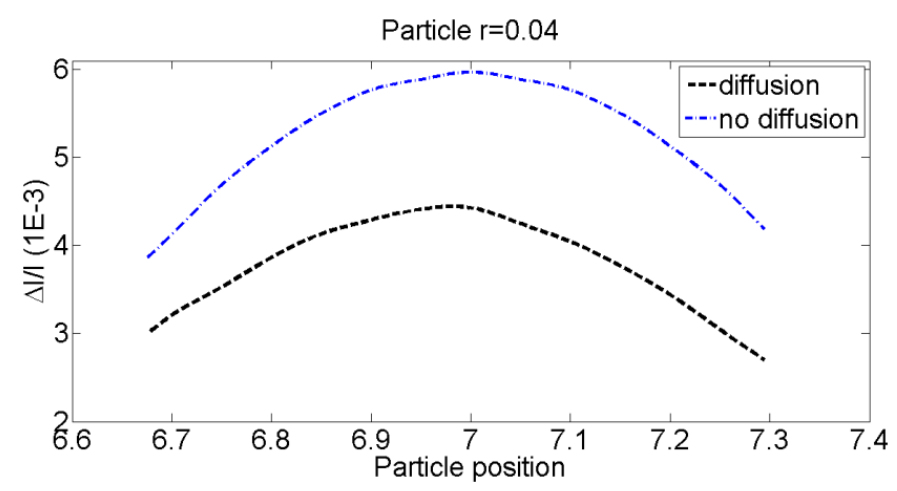

Figure V-3 Comparison of current sensitivity due to the presence of a lowconductivity particle with and without molecular diffusivity.

5.2 The impacts of particle size

To understand the impact of particle size we test thee particles of different radius, the molecular diffusivity coefficient $k=1 e-3$ for all the cases and they have the same sheath/sample stream flow rates. The particle radius is $2 \%, 3 \%$ and $4 \%$ of the channel height, respectively. The impact of particle size is shown in Figure V-4. It is clear that a larger particle leads to higher sensitivity.

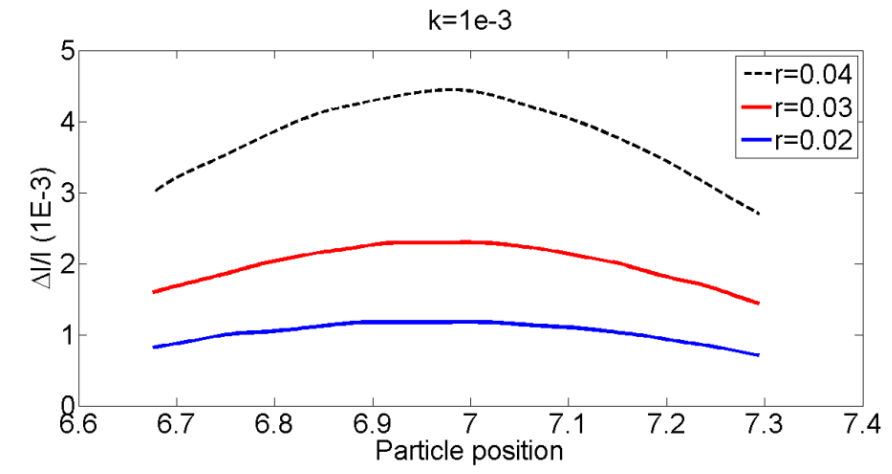

Figure V-4 Impact of particle size on the current sensitivity. A larger particle results in higher sensitivity. 
5.3 The impacts of electrode length and gap between the electrodes

The length of electrodes and the gap between them are two key parameters in the design of Coulter counters. We study their impact by systematically varying the electrode length and the gap. Three different electrodes lengths and three different gaps between the electrodes are tested and the simulation results are shown in Figure V-5. For those tests we find that the sensitivity increases with the electrode length and also with the gap under the selected size variation.
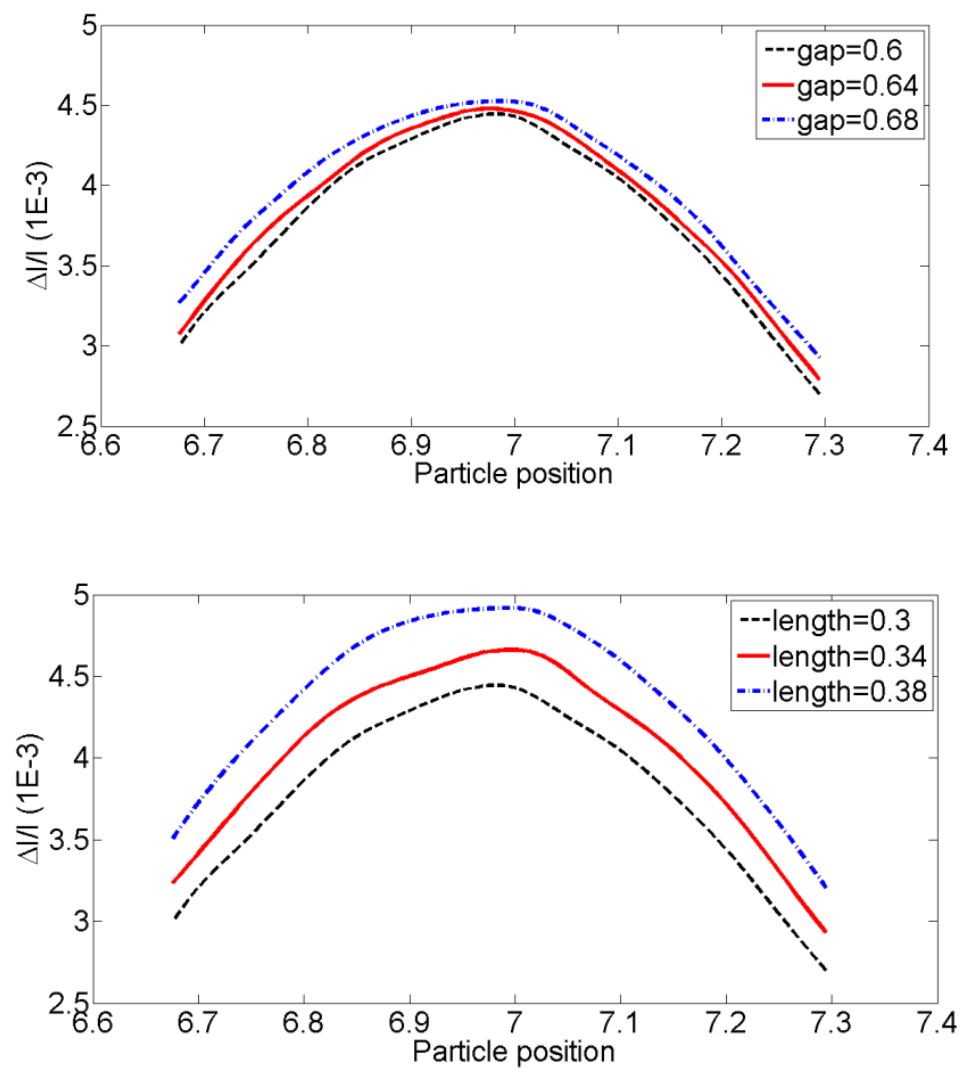

Figure V-5 Variation of current sensitivity with electrode length and gap. Top one is the impact of electrodes gap, bottom one is the impact of electrodes length. 


\subsection{The impacts of electrode position}

The current sensitivity is also affected by the location of the electrodes. Three electrode locations are selected and they are marked in Figure V-6. Here the particle radius is 0.03 for all the tests. In case A, the center is at $\mathrm{x}=6.2$, in case B we move the electrodes further downstream and the center is at $\mathrm{x}=6.6$, in case $\mathrm{C}$, the center is at $\mathrm{x}=7$. In all three cases the gap between the two electrodes is 0.6 . The conductivity distribution at $x=6.2,6.6$ and 7.0 are shown in Figure V-7. Due to the diffusion the conductivity is more spread out further downstream at $x=7.0$ than those at upstream.

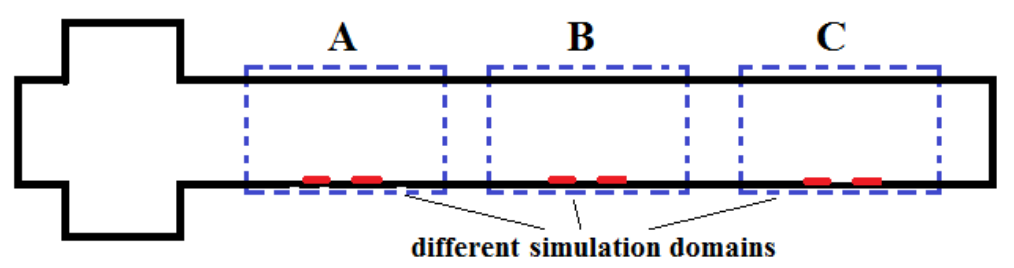

Figure V-6 Three different electrode locations along the channel.

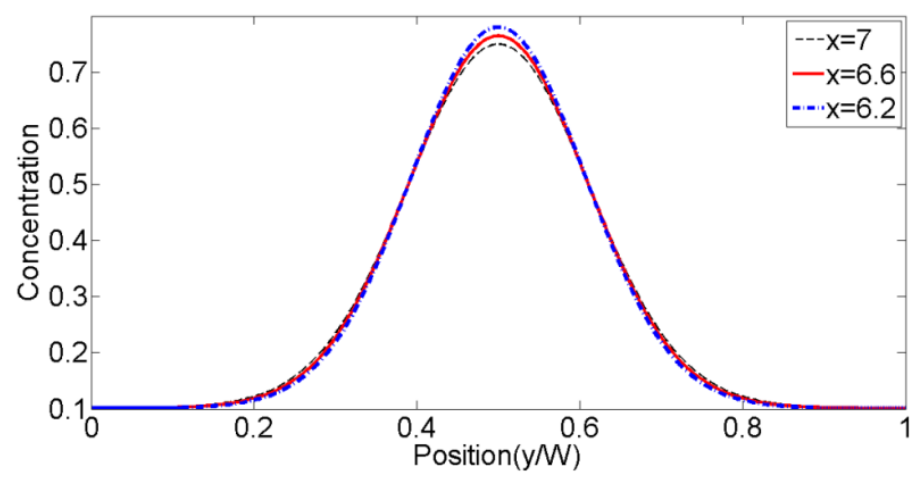

Figure V-7 Cross sectional conductivity distribution at the centers of region A, B and $\mathbf{C}$. 
The current sensitivity is shown in Figure V-8. It shows that when the electrodes are moved downstream, the current sensitivity decreases. This is because the sample stream is more spread out further downstream due to diffusion. Similar to the one shown in Figure V-3, a more concentrated sample stream results in higher current sensitivity than a less concentrated sample stream.

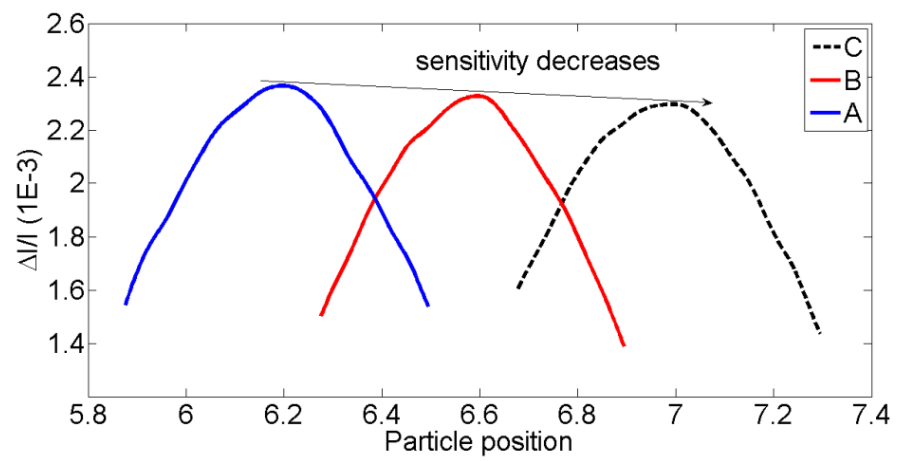

\section{Figure V-8 Impact of electrode position on the current sensitivity.}

\subsection{The impacts of sample flow rate}

The width of the focused sample stream can be adjusted by changing the sample flow rate. A low sheath flow rate leads to a narrow sample stream width and vice versa. Here we choose the tested particle size $\mathrm{r}=0.03$ and three different sheath flow rates ( $Q_{\text {sample }} / Q_{\text {sum }}=0.26, Q_{\text {sample }} / Q_{\text {sum }}=0.28, Q_{\text {sample }} / Q_{\text {sum }}=0.3$ ) to simulate the impact of sample stream width on the current sensitivity. The results are shown in Figure V-9. As the sample stream flow rate decreases, the sample stream width decreases, and the current sensitivity increases. This is because when the sample stream width decreases, the particle affects a relatively large portion of the conductive area and hence the sensitivity is high. 


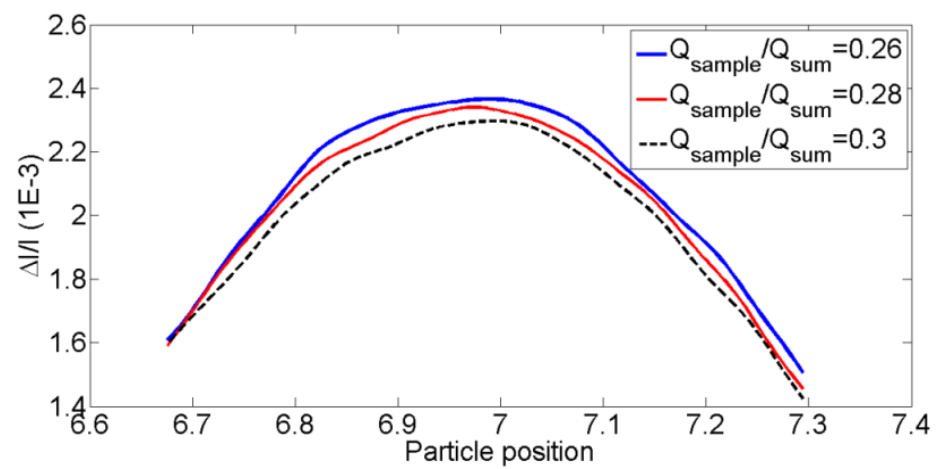

Figure V-9 Impact of the sheath stream flow rate on the current sensitivity.

5.6 The impacts of parallel electrodes distribution

The distribution of the electrodes can all be set as parallel. The electric potential distribution is shown in Figure V-10. Figure V-11 shows the current sensitivity (particle radius $=0.04)$. We can see that the maximum current sensitivity is over $2 \%$ which is nearly five times than the results in Figure V-3(0.4\%). The solution shows the current sensitivity under this parallel distribution is higher than that in the coplanar configuration. This conclusion is also confirmed by Sun et al. (2007).

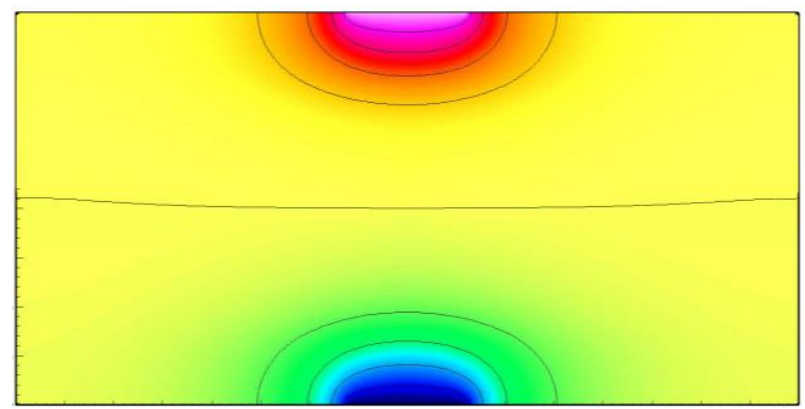

Figure V-10 Electric potential distribution under the parallel electrodes distribution. 


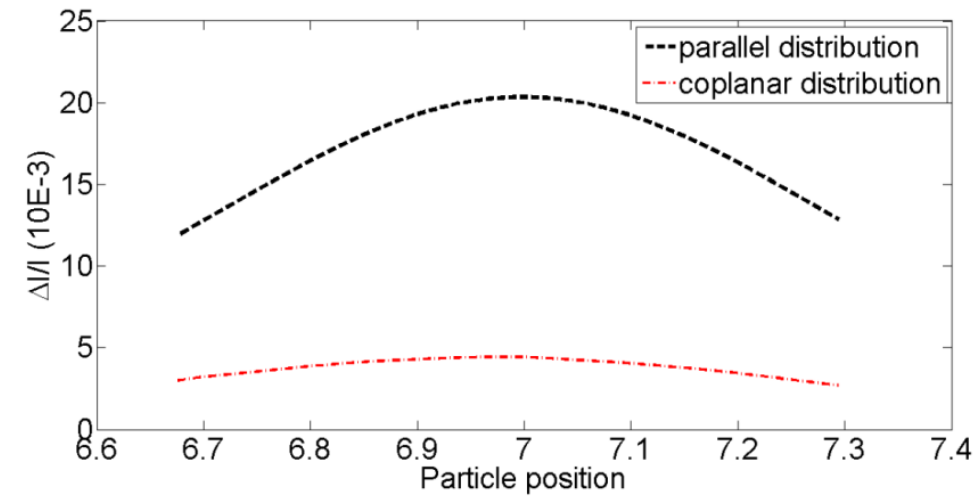

Figure V-11 Current sensitivity under the parallel electrodes distribution.

However, the parallel electrodes distribution is harder to fabricate than the coplanar electrodes distribution. Wood et al. (2007) showed that the parallel configuration needs two times of standard lift-off photo-lithography and sometimes the electrode is easy to fall off. On the other hand, Rodriguez-Trujillo et al. (2007) showed that the fabrication of the coplanar electrodes distribution only needs one-time lithography and there is no electrode problem with falling-off. 


\section{Summary}

We used the CFD method to study the Coulter principle based on a three-inlet microfluidic Coulter counter. The accuracy of the numerical methods was validated by comparing with analytical solutions. Different from some previous work, the mass diffusivity was considered in our work. From our simulations we concluded that diffusion lowers the current sensitivity. Our simulations also showed that the current sensitivity increases with particle size, electrode size, and the gap between the electrodes. We also showed that the sensitivity decreases as the sample stream width increases and as the electrodes are moved further downstream. At last the current sensitivity for the face to face (top and bottom) electrodes configuration is simulated and the merits of the two different electrodes distribution are discussed. 


\section{THREE DIMENSIONAL SIMULATION FOR COULTER PRINCIPLE}

This chapter will study the Coulter principle from the perspective of three dimensional simulation. The three dimensional simulation can give a better view to investigate the signal variation under the impact of the particle. The current signal variation is our research target. In the following paragraph we will study the impacts of particle size, particle position, molecular diffusivity, fluid viscosity, electrode position, electrode length and the gap between the electrodes, detection region location, and sample stream flow rates. At last an entire conclusion will be stated in the summary. 
6.1 The schematic of the simulation domain

The simulation domain is plotted in Figure VI-1. It shows the high-conductive sample flow distribution in a three-inlet channel. Similar as we treated it before, we focus on one region (rectangular area) at the downstream of the micro channel. The size of this region is shown in Figure VI-2. The size of the simulation domain is defined as $L=2 ; W=1 ; H=0.6$.
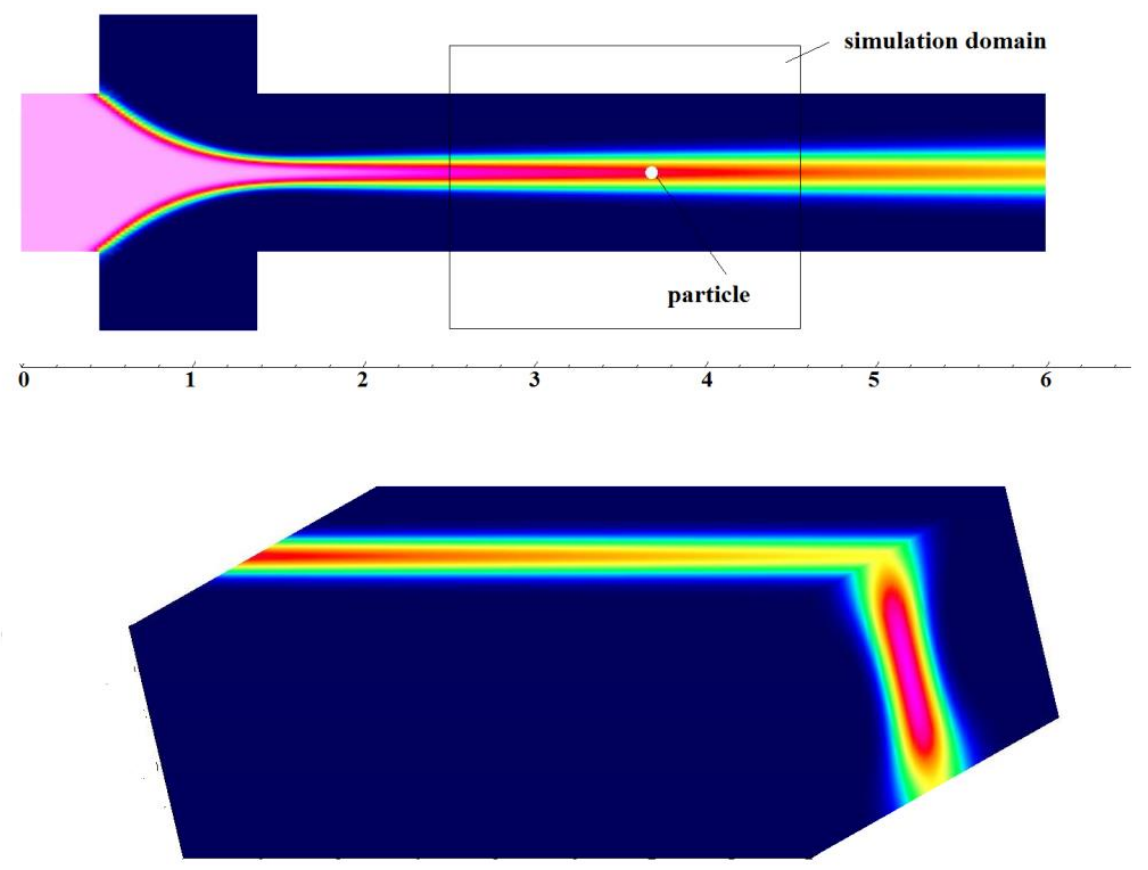

Figure VI-1 The three dimensional simulation domain.

Here the conductivity of the low-conductive sheath stream is 0.1 while the highconductive sample stream is 1 . The default particle motion is horizontal movement along the center symmetry axis of the micro channel. Two electrodes are fixed on the bottom of the simulation domain with symmetry distribution and the same length 
( $L_{11}=0.4, L_{12}=0.7, L_{21}=1.3, L_{22}=1.6$ ). The particle radius is 0.04 compared with the channel width. The conductivity of the particle is 0.1 . The default position of the simulation domain is set from $x=2.5$ to $x=4.5$.

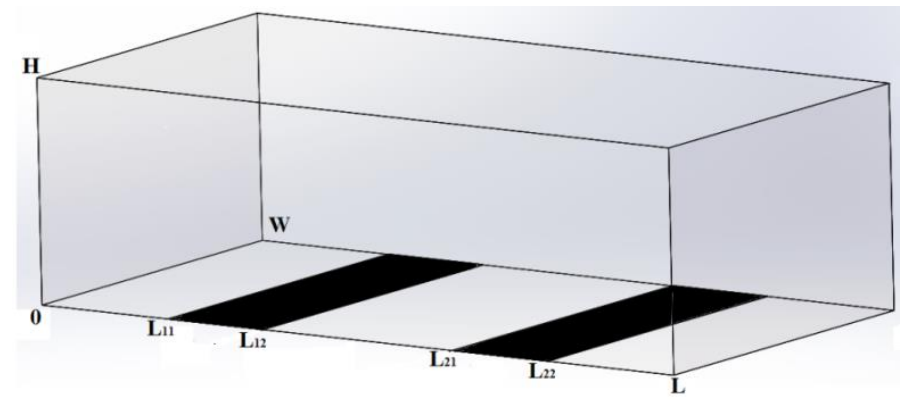

Figure VI-2 The size of the simulation and the electrodes configuration.

6.2 The impacts of Reynolds number

Figure VI-3 shows the impacts of the Reynolds number. We can see that when we increase the Reynolds number from one to ten, the signal sensitivity decreases slightly (the signal sensitivity on the peak deceases $3.6 \%$ ). This can be explained by the ion concentration distribution we talked above, the variation of the Reynolds number from one to ten gives slight influence to the ion concentration distribution. As a result, the signal sensitivity change is very small compared with the variation level of the Reynolds number. 


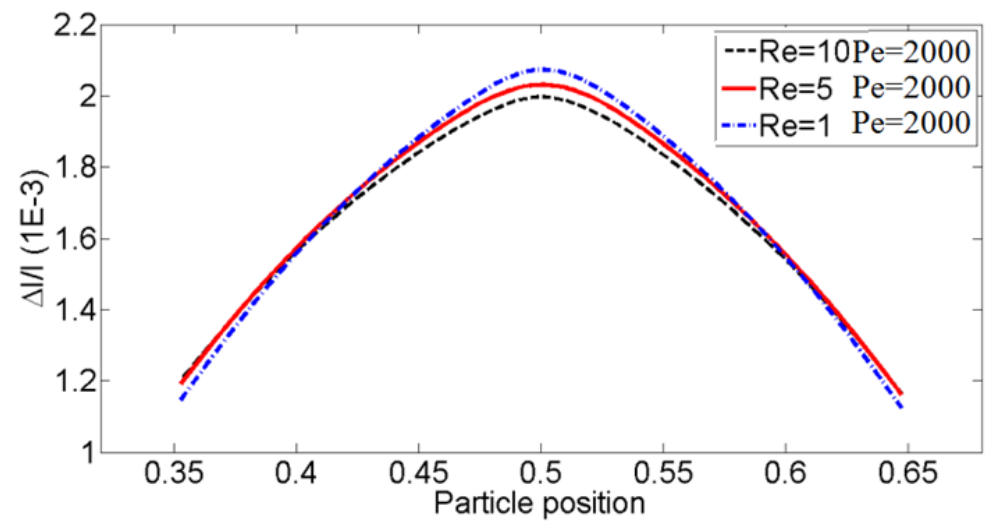

Figure VI-3 Impact of Reynolds number from one to ten on the current sensitivity.

6.3 The impacts of the Peclet number

Figure VI-4 shows the impacts of the Peclet number. We can see that when we decrease the Peclet number, the current sensitivity decreases. This can be explained by looking at the distribution of the current density. Here we extract the current density from the middle

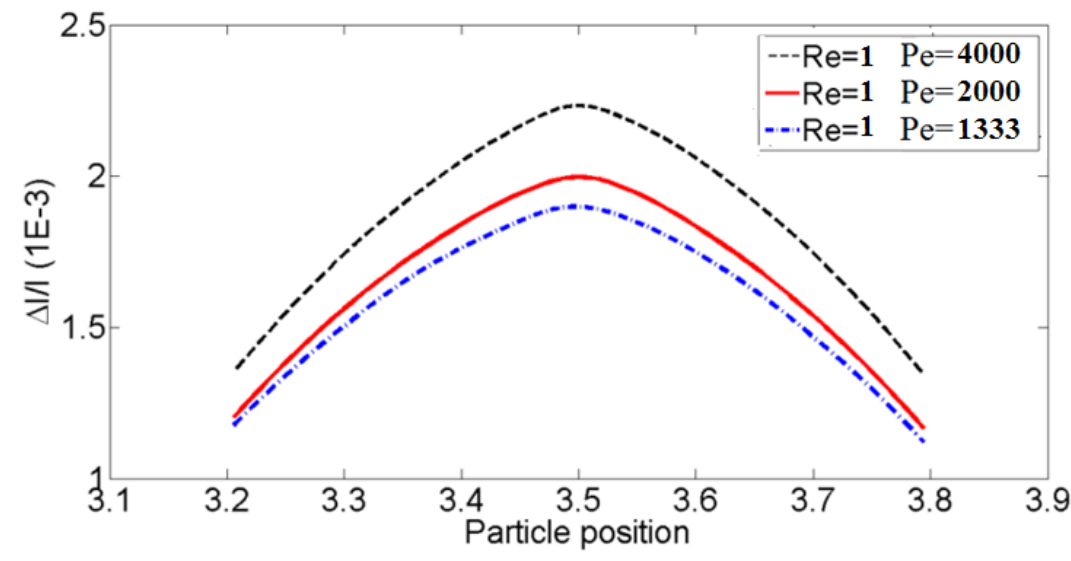

Figure VI-4 Impact of particle size on the current sensitivity. A larger particle results in higher sensitivity. 
cross section of the simulation domain as Figure VI-5 shows. The result from Figure VI-6 shows the current density distribution along this middle cross section without a particle present. We can find that the focused sample stream region has the relative high current density while the sheath stream region has the relative low current density. This is because of the distribution of the conductivity. If we decrease the Peclet number,

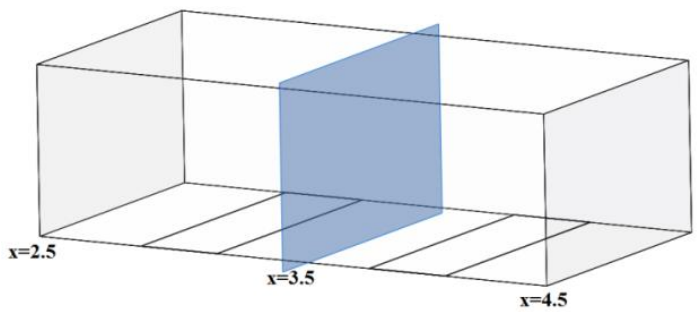

Figure VI-5 Middle cross section.

the conductivity of the sheath stream increases due to the diffusion phenomenon. As a result, more electrons will move through the sheath stream region and their motion will not be affected by the existence of the particle. So the current sensitivity decreases.

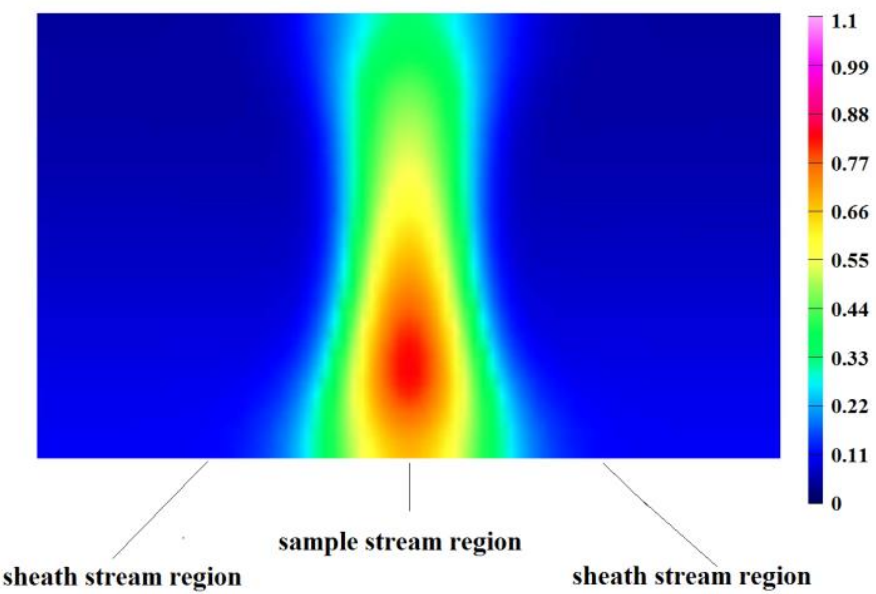

Figure VI-6 Current density distribution on the middle cross section. 
6.4 The impacts of the particle size

Figure VI-7 shows the impacts of the particle size. These three simulation cases are processed under the same Peclet number $\mathrm{Pe}=2000$ and $Q_{\text {sample }} / Q_{\text {sum }}=0.2$. A dimensionless number $f=\frac{r^{2}}{\left(Q_{\text {sample }} / Q_{\text {sum }}\right) W H}$ is defined to reflect the ratio of the particle size and focused sample stream area. We can conclude that the larger $t$, larger particle size and it results in larger current sensitivity due to the enlargement of the particle volume.

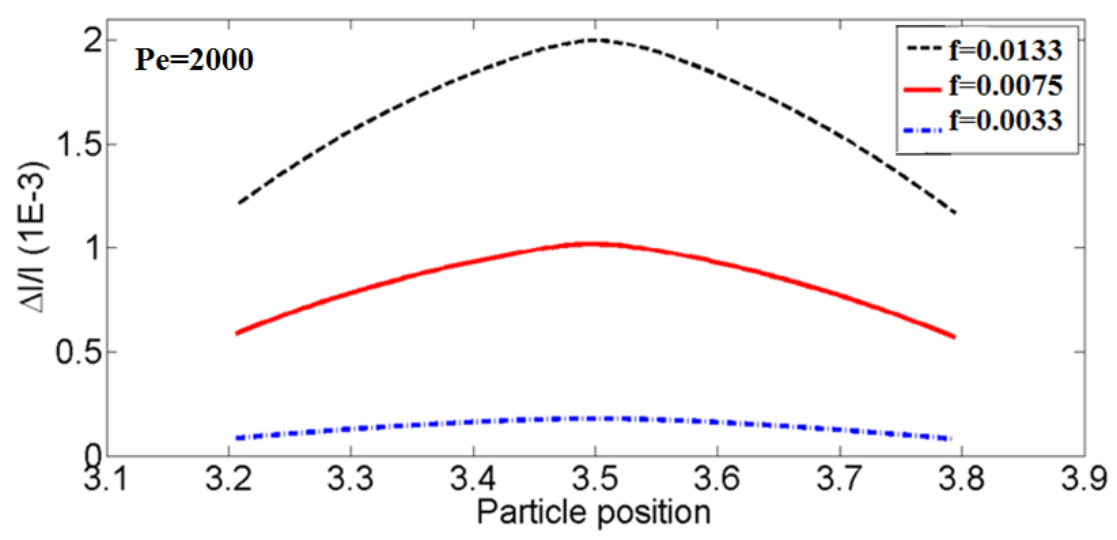

Figure VI-7 Impact of particle size on the current sensitivity.

\subsection{The impacts of the particle position}

In order to investigate the impacts of the vertical particle position, here we simulate three cases with different heights of the particle (the distance between the particle center to the bottom surface of the simulation domain is named particle height). Figure VI-8 shows the results and we can see that the higher of the height, the smaller of the current sensitivity. 
This phenomenon can be understood as the distribution of the current density which is shown in Figure VI-6. We find that the region which is close to the bottom surface edge has relative higher current sensitivity while the region which is far away from the bottom surface edge has relative lower current sensitivity. So it is clear that the case which the particle is close to the bottom edge can affect electrons motion more. As a result, the current sensitivity increases.

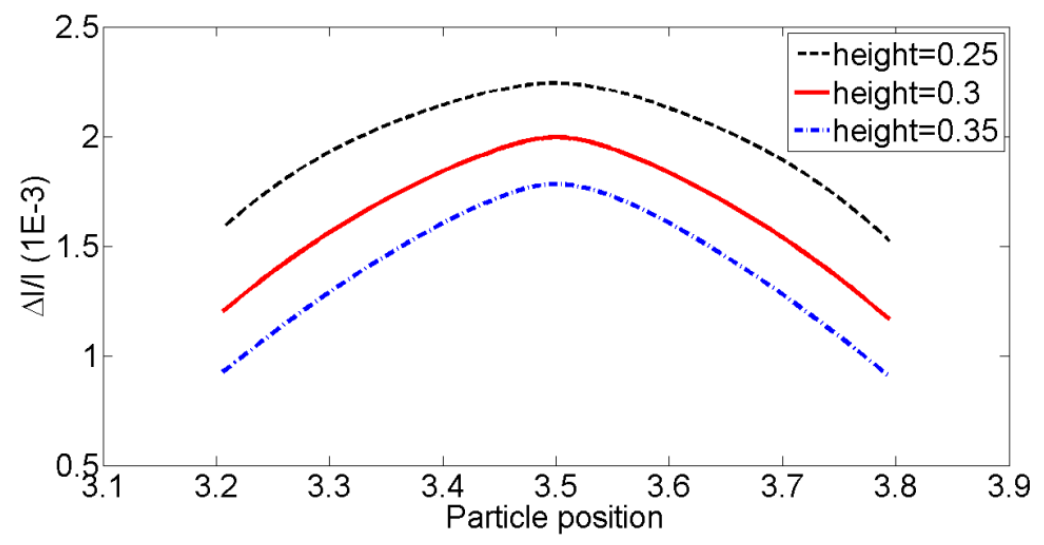

Figure VI-8 Impact of particle height on the current sensitivity.

6.6 The impacts of the sample stream flow rate

The variation of the sample stream flow rate can lead to the variation of the sample stream width. As we tested on the former chapter, the higher sample stream flow rate can achieve larger sample stream width. Here we choose the tested particle size $r=0.04$. Three different sample stream flow rates are tested $\left(Q_{\text {sample }} / Q_{\text {sum }}=0.2, Q_{\text {sample }} / Q_{\text {sum }}=0.25\right.$ and $Q_{\text {sample }} / Q_{\text {sum }}=0.3$ ) to simulate the impact of sample stream flow rate on the current sensitivity. 


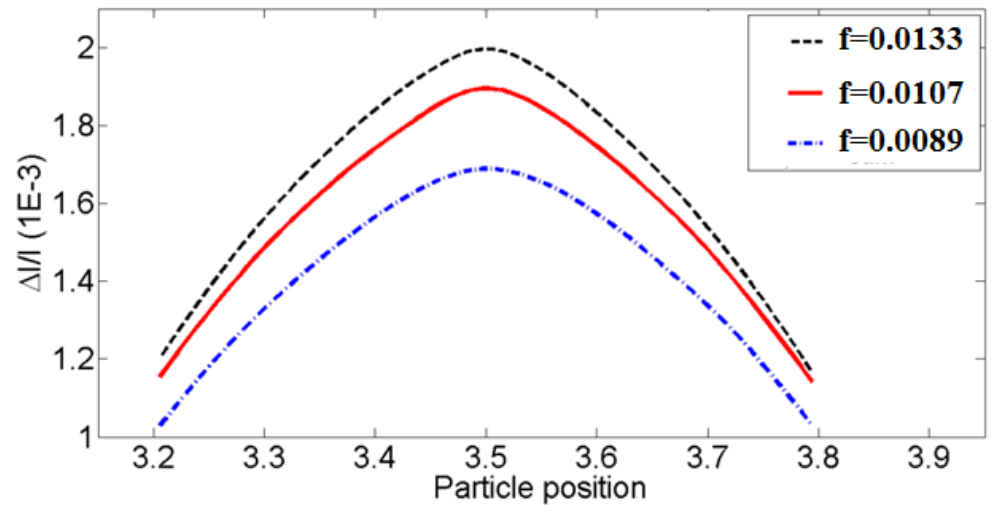

Figure VI-9 Impact of sample stream flow rates on the current sensitivity.

The results are shown in Figure VI-9. As the sample stream flow rates increases, the dimensionless number f decreases, the current sensitivity decreases. This is because when $\mathrm{f}$ decreases, the particle affects a relatively smaller portion of the conductive area and hence the sensitivity is relative lower.

\subsection{The impacts of the electrodes length/gap}

Here we use the similar method which we chose to deal with in the two dimensional simulation. We systematically vary the electrode length and the gap. Three different

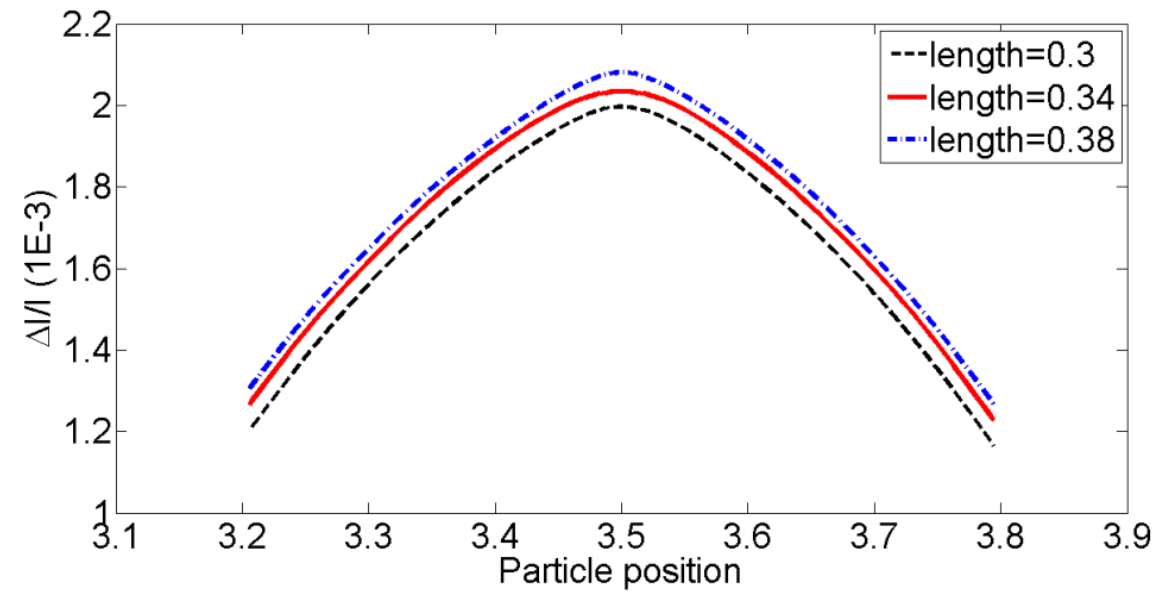




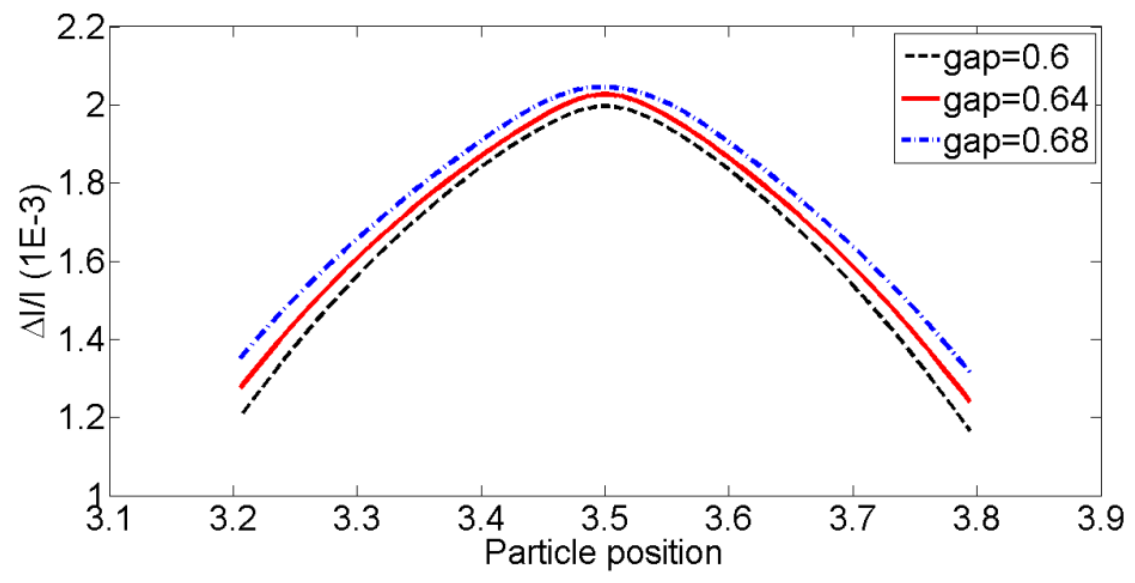

Figure VI-10 Impact of electrodes length and gap between the electrodes.

electrode lengths and three different gaps between the electrodes are tested and the simulation results are shown in Figure VI-10. For those tests we conclude that the sensitivity increases with the electrode length and also with the gap.

\subsection{The impacts of the electrodes position}

As we discussed in two dimensional simulation, the current sensitivity is also affected by the location of the electrodes. Here three different detection regions are selected and they are shown in Figure VI-11. The particle radius is 0.04 for all the tests. In case A, the simulation domain is $X_{0}=2.5, X_{1}=4.5$; in case B we move the electrodes further downstream and the domain is $X_{0}=3, X_{1}=5$, in case C, the domain is $X_{0}=3.5, X_{1}=5.5$. In all three cases the gap between the two electrodes is fixed as 0.6. 


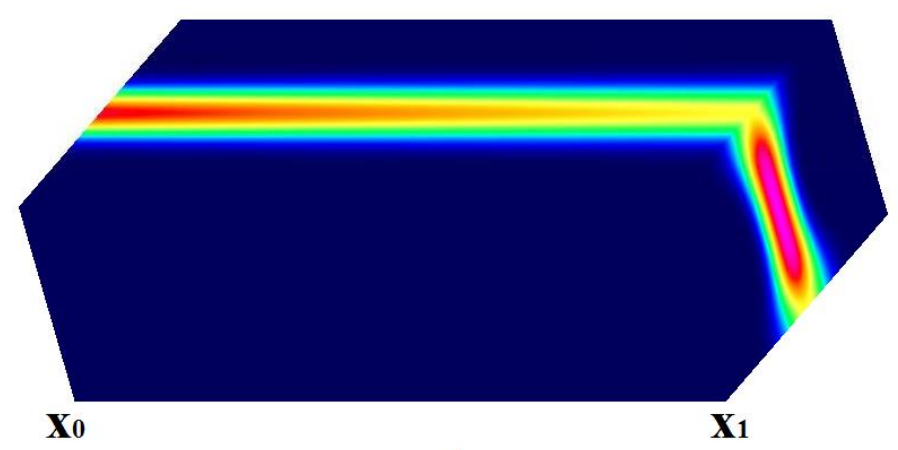

$\operatorname{caseA}$
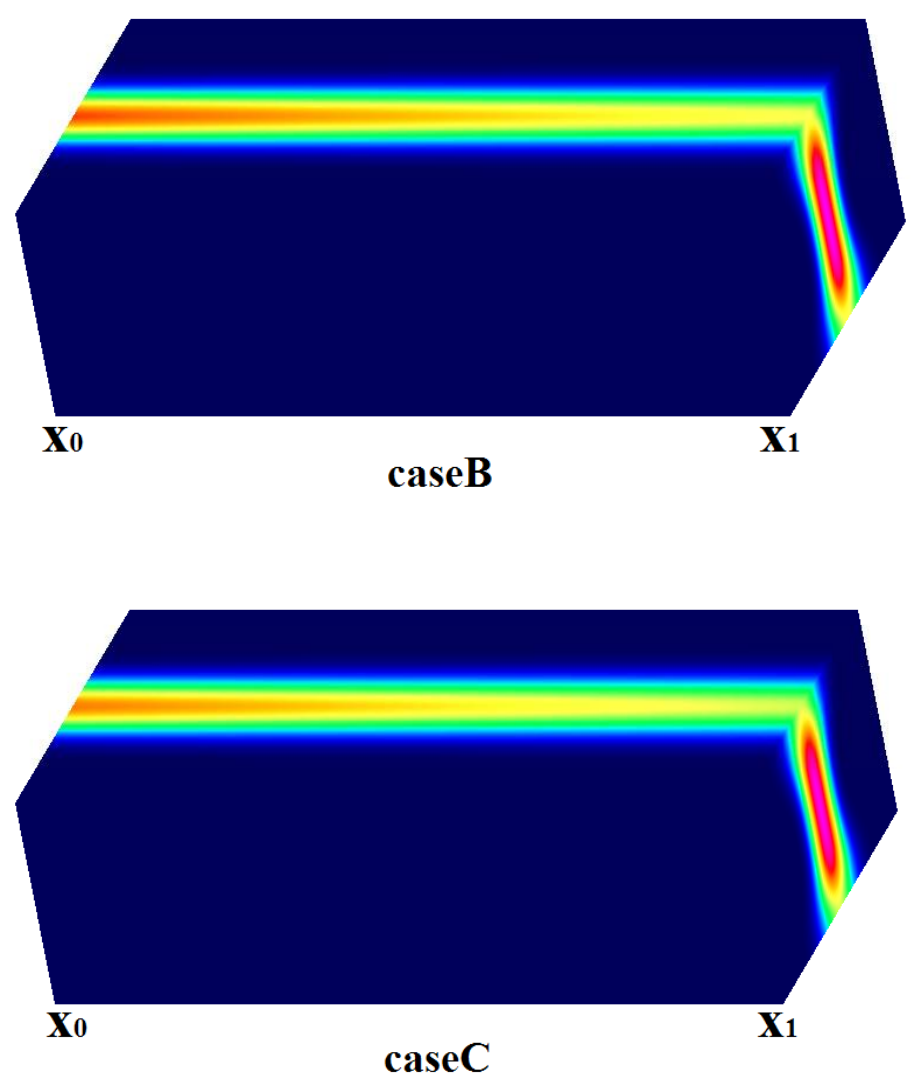

Figure VI-11 Three different positions of simulation domain.

The current sensitivity variation simulation result is shown in Figure VI-12. It shows that when the electrodes are moved downstream, the current sensitivity decreases. This conclusion is the same as we got from the two dimensional simulation about the impact 
of the molecular diffusivity. A sample stream with more concentrated concentration distribution results in higher current sensitivity than one with less concentrated concentration distribution.

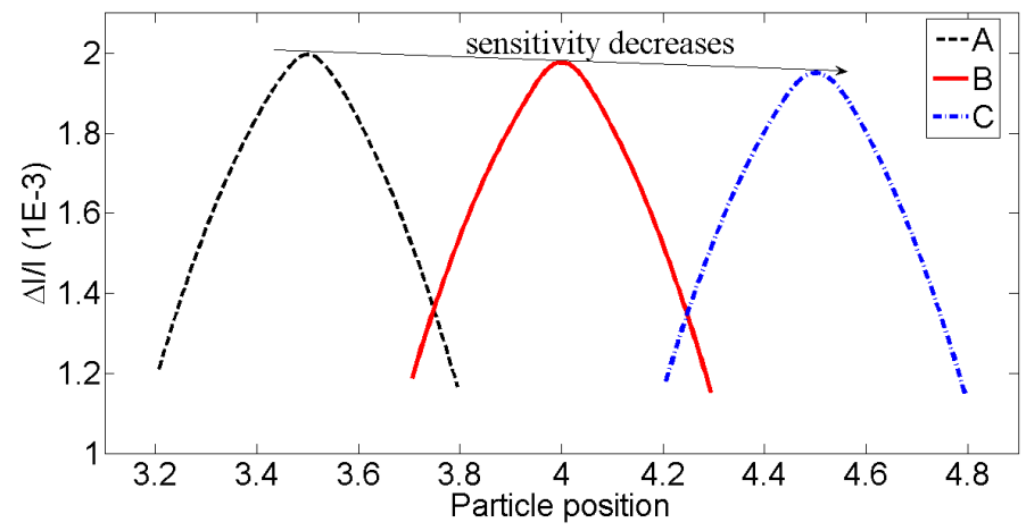

Figure VI-12 Impact of electrodes position translation.

6.9 The impacts of parallel electrodes distribution

Here we also simulate the current variation under the impact of the parallel electrodes distribution. Two cases with different electrodes distribution are shown in Figure VI-13.

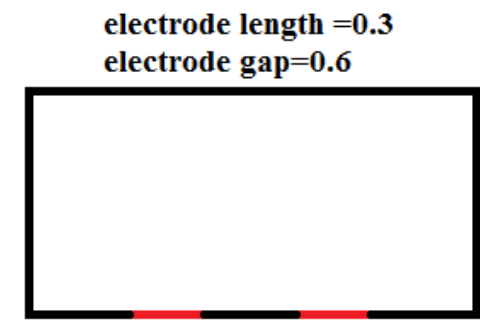

coplanar distribution

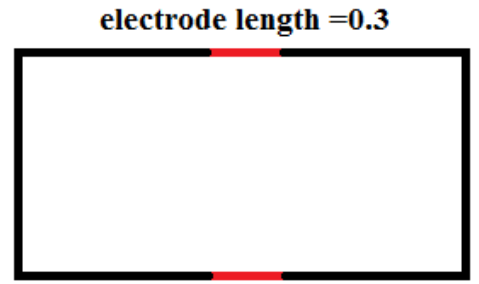

parallel distribution

Figure VI-13 Two different electrode distributions. 
The current variation results are shown in Figure VI-14. We can see that the max current variation in the case of parallel electrode distribution is larger than the max current variation in the case of coplanar electrode distribution. This conclusion is the same as we get from the two dimensional simulation.

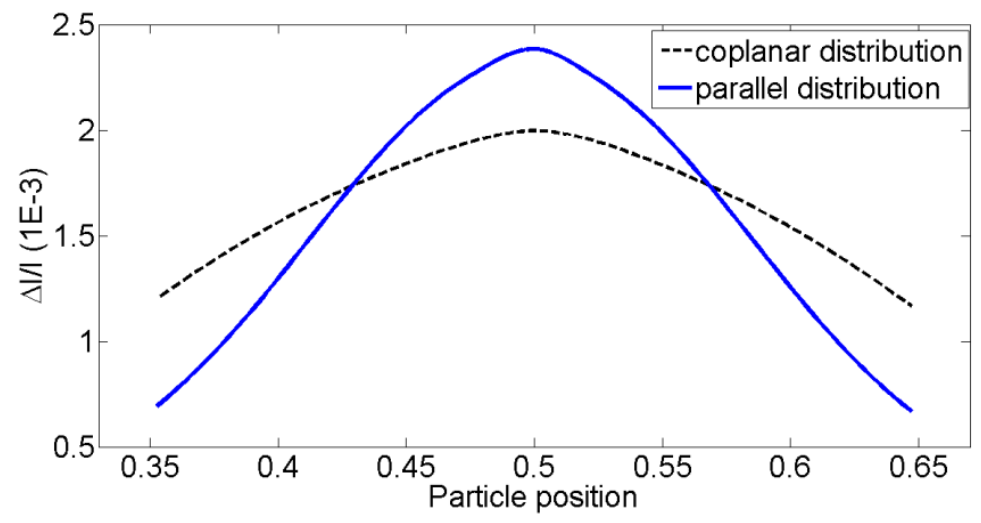

Figure VI-14 The comparison of two different electrodes distributions. 


\section{Summary}

This chapter has a detailed investigation about the Coulter principle under the impacts of the hydrodynamic focusing by 3D simulation. The impacts of several physical/design parameters are discussed. Based on our simulation results, we conclude that the larger molecular diffusivity coefficient can lead to a smaller current sensitivity due to the leak of the effective electrical conductivity. The larger the particle size, the larger current sensitivity because of the increasing of the blocking ability. For the impacts of the electrodes length and the gap between the electrodes, the results show that increasing the electrodes length and gap can get better current sensitivity under the domain size we choose; The particle position tests show that under the condition of the coplanar distribution of electrodes, decreasing the displacement between the particle to the bottom surface edge of the simulation domain can lead to a larger current sensitivity because the motion of more electrons are effected; The simulation for the electrodes position variation shows that the current sensitivity decreases as the electrodes moves towards downstream. The tests for the sample stream flow rates cases confirm that the increasing of the sample stream flow rates will decrease the current sensitivity because the effective portion of the particle over the detection domain is decreased. At last a comparison for two different electrodes configuration is simulated and the result shows that the parallel electrodes configuration can achieve better current sensitivity. 


\section{CONCLUSION AND FUTURE WORK}

In this section, a short summary about the work is given and the results are collected together with suggested improvements and targets of interest for future research. The summary will illustrate the dissertation background, the governing equation, the numerical simulation methods, and the results which are mentioned above. At last the summary will conclude with a discussion of the future work on the simulation aspect of this field. 


\subsection{Background}

First, the dissertation shows two different kinds of particle detection methods: light optical scattering and Coulter counter principle. The mechanism of each method is introduced. After that the Coulter counter principle is investigated and two important factors (hydrodynamic focusing, electrical field configuration with signal response) are introduced including the experiment designs, theorems, and numerical simulations. It points out that though many new novel designs of micro Coulter counter are invented; the details of the hydrodynamic focusing performance and its impacts to the DC detection method still need to be studied fundamentally. Especially the cases that ion concentration distribution and particle motion in micro channel are not been studied in details. Several difficult points at the numerical simulation aspects are discussed.

\subsection{Governing equation and numerical method}

Navier-Stokes and convection-diffusion equations are used to solve the ion concentration distribution; Laplace equation is used to solve the electrical potential distribution. In order to solve the particle motion, a grid meshing named "overlap mapping" is introduced based on the solver named "Overture". Then the numerical difference methods are discussed.

\subsection{Results and discussion}

The performances of the hydrodynamic focusing were discussed. Both two dimensional and three dimensional simulation results showed us that by fixing the Peclet number, the 
variation of the Reynolds number from one to ten has slight influence to the ion concentration distribution while smaller Reynolds number can get smoother concentration distribution if we fix the Schmidt number. Also, a larger sample stream flow rate can achieve larger ion concentration values because more ions enter into the channel at the same time period. Meanwhile the simulation results showed that the concentration distribution changed negligibly when the Reynolds number was from one to ten. The asymmetric flow rate situation was researched. During the two dimensional simulation, an analytical formula was depicted from the mass conservation theorem. The simulated offsets about the concentration peak to the middle axis matched well with the analytical solution. During the three dimensional simulation, the impacts of three different parameters (sample stream flow rate, sheath stream flow rates difference, micro channel height) were investigated. The sample stream flow rate did not affect the concentration peak bias while the other two parameters did.

The impacts of the hydrodynamic focusing on the particle motion were also tested. The results showed that the hydrodynamic focusing can increase the particle gap on the horizontal direction but push the particle into the middle region on the vertical direction. Multiple cases both on two dimensional and three dimensional simulation were tested to show that decreasing the sample stream flow rate can enlarge the horizontal gap between particles.

After that, the signal variation situation of micro Coulter counter principle based on a three-inlet microfluidic Coulter counter was simulated. The simulation considered the impacts of the hydrodynamic focusing. From our results we found that the signal 
sensitivity did not change significantly when the Reynolds number was from one to ten due to the reason of the ion concentration distribution as previously discussed. The diffusion phenomenon decreased the current signal sensitivity because it decreased the current density at the detection region (ion motion from the sample stream to the sheath stream). Also, the current sensitivity increased with particle size, electrode length, and the gap between the electrodes under the selected size variation, but decreased as the sample stream flow rate increased. Furthermore, the current sensitivity decreased when the electrodes moved further downstream (similar reason which was used to explain the impacts of the diffusion phenomenon). Lastly, we performed an investigation about the impacts of the particle height position. It showed that a closer particle location to the bottom electrodes can achieve a better current sensitivity.

In sum, this dissertation gave a relatively detailed study on the numerical simulation of the micro Coulter counter which uses hydrodynamic focusing. The total process covered the simulations about fluid field, concentration field, particle motion and electrical potential field. The impacts of the hydrodynamic focusing were investigated from the aspects of ion concentration distribution, particle motion and current sensitivity variation. The numerical results showed that the hydrodynamic focusing can improve the current sensitivity while the molecular diffusivity phenomenon can decrease it. Meanwhile, the impacts of several design parameters such as molecular diffusivity, sample stream flow rate, particle size, particle position and electrodes configuration were considered in our simulation. 


\subsection{Future work}

Though this dissertation is a relatively detailed study on the working mechanism and performances of the Coulter counter principle and the impacts of hydrodynamic focusing, there are still some interesting or difficult points which deserve further research. The following paragraphs list parts of them.

First, for particle detection, a DC power supply can only give the information of cell size so people try to use AC power supply to find additional information of the particles. The basic principle of AC power supply, also known as impedance spectroscopy (IS), is to measure the dielectric properties of a system by applying an $\mathrm{AC}$ power excitation signal and measuring the response signal (Cole KS (1941), Pauly and Schwan (1959)). The numerical simulation of the AC detection method can give us more and better information about the particle information. Several cases are done with no impacts of hydrodynamic focusing (Ito et al. (2003), Wu et al. (2010) and Richards et al. (2012)). More work can be focused in this field from the numerical simulation aspect by considering the hydrodynamic focusing and molecular diffusivity phenomenon.

Another topic is considering the particle motion under the impacts of the electrical force. Right now we assume that the particle is neutral and is not affected by the electrical force. Some experiments show that the polarization phenomenon exists in some certain environments. In those cases the external electrical force term needs to be added to the Navier-Stokes equation. Also the simulation of the ion concentration distribution needs to consider the impact of the electrical force, so convection-diffusion equation is not 
available any more, instead, a new equation named Nernst-Planck equation (Gjelstad et al. (2007), Belova et al. (2007)) is needed to solve it. Theoretically speaking, solving the Navier-Stokes equation, Nernst-Planck equation and Laplace equation together can give better results to investigate the performance of micro Coulter counter. However, the computation for the coupling of these three equations is really large, the transient particle motion simulation is worse to finish. Such simulations are rarely found from published papers. Considering the fast development of the high-performance computer, it can be a break in the future that finds out more detail information by numerical simulation with the help of new updated computing device. 


\section{REFERENCES}

[1] Ashish, V.J., Jiang, Zhe, Jun, Hu and Joan, Carletta (2006). Detection and counting of micro-scale particles and pollen using a multi-aperture Coulter counter. Measurement Science and Technology 17.

[2] Ateya, D.A., Erickson, J.S., Howell, P.B., Jr., Hilliard, L.R., Golden, J.P., and Ligler, F.S. (2008). The good, the bad, and the tiny: a review of microflow cytometry. Analytical and bioanalytical chemistry 391, 1485-1498.

[3] Belova, I.V., Allnatt, A.R., and Murch, G.E. (2007). Interdiffusion in strongly ionic insulating compounds: The Nernst-Planck equation. Philosophical Magazine 87, 4169-4180.

[4] Berge, L.I., Jossang, T., and Feder, J. (1990). Off-Axis Response for Particles Passing through Long Apertures in Coulter-Type Counters. Measurement Science \& Technology 1, 471-474.

[5] Berger, A., Talbot, L, Yao LS (1983). Flow in curved pipes. Annual review of fluid mechanics 15, 461-512.

[6] Bernabini, C., Holmes, D., and Morgan, H. (2011). Micro-impedance cytometry for detection and analysis of micron-sized particles and bacteria. Lab on a chip 11, 407412.

[7] Blonski, S., Domagalski, P., Dziubinski, M., and Kowalewski, T.A. (2011). Hydrodynamically modified seeding for micro-PIV. Archives of Mechanics 63, 163-182.

[8] Brown, D., Henshaw WD, Quinlan DJ (1997). Overture: An Object-oriented Framework for Solving Partial Differential Equations. Computer Science 1343, 177184. 
[9] Chapman, M.R., and Sohn, L.L. (2011). Label-free resistive-pulse cytometry. Methods in cell biology 102, 127-157.

[10]Chaudhry, M.A., and Schinzinger, R. (1992). Numerical Computation of the Schwarz-Christoffel Transformation Parameters for Conformal Mapping of Arbitrarily Shaped Polygons with Finite Vertices. Compel-the International Journal for Computation and Mathematics in Electrical and Electronic Engineering 11, 263275.

[11]Chih-Chang, C., Zhi-Xiong, Huang and Ruey-Jen, Yang (2007). Three-dimensional hydrodynamic focusing in two-layer polydimethylsiloxane (PDMS) microchannels. J Micromech Microeng 17, 1479-1486.

[12]Cho, S. (2009). Electrical resistance characterization of insulating membrane with multipore. Journal of Electroanalytical Chemistry 629, 69-72.

[13]Chung, T.D., and Kim, H.C. (2007). Recent advances in miniaturized microfluidic flow cytometry for clinical use. Electrophoresis 28, 4511-4520.

[14]Cole KS, C.R. (1941). Dispersion and Absorption in Dielectrics I. Alternating Current Characteristics. Journal of Chemical Physics 9.

[15]Cowan, M.P., and Harfield, J.G. (1990). The Linearity and Response of Focussed Apertures. Particle \& Particle Systems Characterization 7, 1-5.

[16]Cunningham, A., and Buonnacorsi, G.A. (1992). Narrow-angle forward light scattering from individual algal cells: implications for size and shape discrimination in flow cytometry. Journal of Plankton Research 14, 223-234.

[17]DeBlois, R., Bean CP (1970). Counting and Sizing of Submicron Particles by the Resistive Pulse Technique. Review of Scientific Instruments 41.

[18]Delillo, T.K., and Kropf, E.H. (2011). Numerical Computation of the SchwarzChristoffel Transformation for Multiply Connected Domains. Siam Journal on Scientific Computing 33, 1369-1394. 
[19]Domagalski, P.M. (2011). Hydrodynamic focusing of liquid in intersection of microchannels. (Lodz, Poland, Lodz Technical University).

[20]Dubelaar, G.B., Gerritzen, P.L., Beeker, A.E., Jonker, R.R., and Tangen, K. (1999). Design and first results of CytoBuoy: a wireless flow cytometer for in situ analysis of marine and fresh waters. Cytometry 37, 247-254.

[21]Gawad, S., Schild, L., and Renaud, P. (2001). Micromachined impedance spectroscopy flow cytometer for cell analysis and particle sizing. Lab on a chip 1, 76-82.

[22]Gjelstad, A., Rasmussen, K.E., and Pedersen-Bjergaard, S. (2007). Simulation of flux during electro-membrane extraction based on the Nernst-Planck equation. Journal of chromatography. A 1174, 104-111.

[23]Grégoire, A. (2007). Numerical Analysis and Optimization: An Introduction to Mathematical (New York, USA: Oxford).

[24]Gregg, E.C., and Steidley, K.D. (1965). Electrical counting and sizing of mammalian cells in suspension. Biophys J 5, 393-405.

[25]Guo, J.H., Pui, T.S., Rahman, A.R.A., and Kang, Y.J. (2012). Numerical Investigation of the Performance of Coulter Counter with Novel Structure. International Journal of Information and Electronics Engineering 2.

[26]Hairer, G., Pärr, GS , Svasek, P, Jachimowicz, A, and Vellekoop, MJ (2007). A Novel coaxial sheath flow device for sample focusing. In Eleventh International Conference on Miniaturized Systems for Chemistry and Life Sciences (Paris, France, Chemical and Biological Microsystems Society).

[27]Hashemi, N., Howell, P.B., Jr., Erickson, J.S., Golden, J.P., and Ligler, F.S. (2010). Dynamic reversibility of hydrodynamic focusing for recycling sheath fluid. Lab on a chip 10, 1952-1959.

[28]Henshaw, W. (1994). A Fourth-Order Accurate Method for the Incompressible Navier-Stokes Equations on Overlapping Grids. Journal of Computational Physics $113,13-25$. 
[29]Herzenberg, L.A., Parks, D., Sahaf, B., Perez, O., Roederer, M., and Herzenberg, L.A. (2002). The history and future of the fluorescence activated cell sorter and flow cytometry: a view from Stanford. Clin Chem 48, 1819-1827.

[30]Hoffman, J.D. (2001). Numerical methods for engineers and scientists, 2nd edn (New York: Marcel Dekker).

[31]Hou, H.H., Tsai, C.H., Fu, L.M., and Yang, R.J. (2009). Experimental and numerical investigation into micro-flow cytometer with 3-D hydrodynamic focusing effect and micro-weir structure. Electrophoresis 30, 2507-2515.

[32] Howard, M.S. (2005). Practical Flow Cytometry (John Wiley \& Sons).

[33]Howell, P.B., Jr., Golden, J.P., Hilliard, L.R., Erickson, J.S., Mott, D.R., and Ligler, F.S. (2008). Two simple and rugged designs for creating microfluidic sheath flow. Lab on a chip 8, 1097-1103.

[34]Huh, D., Gu, W., Kamotani, Y., Grotberg, J.B., and Takayama, S. (2005). Microfluidics for flow cytometric analysis of cells and particles. Physiol Meas 26, R73-98.

[35]Ito, T., Sun, L., and Crooks, R.M. (2003). Simultaneous determination of the size and surface charge of individual nanoparticles using a carbon nanotube-based Coulter counter. Analytical chemistry 75, 2399-2406.

[36]Ito, T., Sun, L., Henriquez, R.R., and Crooks, R.M. (2004). A carbon nanotube-based coulter nanoparticle counter. Acc Chem Res 37, 937-945.

[37]Ivorra, B., Redondo, J.L., Santiago, J.G., Ortigosa, P.M., and Ramos, A.M. (2013). Two- and three-dimensional modeling and optimization applied to the design of a fast hydrodynamic focusing microfluidic mixer for protein folding. Physics of Fluids 25, 032001-032017.

[38]Jiang, L., Wang, W., Chau, Y., and Yao, S. (2013). Controllable formation of aromatic nanoparticles in a three-dimensional hydrodynamic flow focusing microfluidic device. RSC Advances 3, 17762-17769. 
[39]Kawaguchi, C., Noda, T., Tsutsui, M., Taniguchi, M., Kawano, S., and Kawai, T. (2012). Electrical detection of single pollen allergen particles using electrodeembedded microchannels. Journal of Physics-Condensed Matter 24.

[40]Kerker, M. (1969). CHAPTER 7 - Analysis of Particle Size. In The Scattering of Light and Other Electromagnetic Radiation, M. Kerker, ed. (Academic Press), pp. 311-413.

[41]Kerker, M. (1983). Elastic and inelastic light scattering in flow cytometry. Cytometry $4,1-10$.

[42]Kersaudy-Kerhoas, M., Dhariwal, R., and Desmulliez, M.P. (2008). Recent advances in microparticle continuous separation. IET nanobiotechnology / IET 2, 1-13.

[43]Kobayashi, Y., and Martin, C.R. (1997). Toward a molecular Coulter® counter type device. Journal of Electroanalytical Chemistry 431, 29-33.

[44]Kulrattanarak, T., van der Sman, R.G., Schroen, C.G., and Boom, R.M. (2008). Classification and evaluation of microfluidic devices for continuous suspension fractionation. Adv Colloid Interface Sci 142, 53-66.

[45]Kummrow, A., Theisen, J., Frankowski, M., Tuchscheerer, A., Yildirim, H., Brattke, K., Schmidt, M., and Neukammer, J. (2009). Microfluidic structures for flow cytometric analysis of hydrodynamically focussed blood cells fabricated by ultraprecision micromachining. Lab on a chip 9, 972-981.

[46]Kunstmann-Olsen, C., Hoyland, J., and Rubahn, H.-G. (2012). Influence of geometry on hydrodynamic focusing and long-range fluid behavior in PDMS microfluidic chips. Microfluidics and Nanofluidics 12, 795-803.

[47]Lee, G., Chang CC, Huang SB, Yang RJ (2006). The hydrodynamic focusing effect inside rectangular microchannels. J Micromech Microeng 16, 1024-1032.

[48]Lee, G., Huang CI, Ke BJ, Huang GR, Hwei BH, Lai HF (2001). Hydrodynamic Focusing for a Micromachined Flow Cytometer. Journal of Fluids Engineering 123, 672-679. 
[49]Lee, M.G., Choi, S., and Park, J.K. (2009). Three-dimensional hydrodynamic focusing with a single sheath flow in a single-layer microfluidic device. Lab on a chip 9, 3155-3160.

[50]Lee, S., Izuo SI, Inatomi KI (2004). A CAD study on micro flow cytometer and its application to bacteria detection. IEEE Sensors 1, 308-311.

[51]Li, J., and Renardy, Y. (2000). Numerical Study of Flows of Two Immiscible Liquids at Low Reynolds Number. SIAM Review 42, 417-439.

[52]Lin, S.C., Yen, P.W., Peng, C.C., and Tung, Y.C. (2012). Single channel layer, single sheath-flow inlet microfluidic flow cytometer with three-dimensional hydrodynamic focusing. Lab on a chip 12, 3135-3141.

[53]MADHURI, K. (2007). A mems based Coulter counter for cell sizing. (Columbia, MO, University of Missouri-Columbia).

[54]Mao, X., Waldeisen, J.R., and Huang, T.J. (2007). "Microfluidic drifting"-implementing three-dimensional hydrodynamic focusing with a single-layer planar microfluidic device. Lab on a chip 7, 1260-1262.

[55]Maxwell, J.C., and Thomson, J.J. (1904). A treatise on electricity and magnetism, 3d edn (Oxford,: Clarendon).

[56]Munson BR, Y.D., Okiishi TH, Huebsch WW (2009). Fundamentals of Fluid Mechanics, 6 edn (Wiley).

[57]Narayan, S., Michael, SP, Luke, PL, and Andrew, AB (2004). Three-Dimensional Hydrodynamic Focusing in Polydimethylsiloxane (PDMS) Microchannels. Journal of microelectromechanical systems 13, 559-567.

[58]Nieuwenhuis, J.H., Kohl, F., Bastemeijer, J., Sarro, P.M., and Vellekoop, M.J. (2004). Integrated Coulter counter based on 2-dimensional liquid aperture control. Sensor Actuat B-Chem 102, 44-50.

[59]Pamme, N. (2007). Continuous flow separations in microfluidic devices. Lab on a chip 7, 1644-1659. 
[60]Pauly, H., and Schwan, H.P. (1959). [Impendance of a suspension of ball-shaped particles with a shell; a model for the dielectric behavior of cell suspensions and protein solutions]. Z Naturforsch B 14B, 125-131.

[61]Piotr, M., Marek, D, Pawel, B, Michal, MM, Lars RS (2007). Width variations of hydrodynamically focused streams in low to moderate Reynolds number. European Congress of Chemical Engineering.

[62]Qin, Z. (2009). Modeling of Ion Transport for Micro/Nano Size Particles in Coulter Counter Application. In Mechanical Engineering (Ohio, University of Akron).

[63]Qin, Z.P., Zhe, J.A., and Wang, G.X. (2011). Effects of particle's off-axis position, shape, orientation and entry position on resistance changes of micro Coulter counting devices. Measurement Science \& Technology 22.

[64]Rawstron, A.C., Child, J.A., de Tute, R.M., Davies, F.E., Gregory, W.M., Bell, S.E., Szubert, A.J., Navarro-Coy, N., Drayson, M.T., Feyler, S., et al. (2013). Minimal Residual Disease Assessed by Multiparameter Flow Cytometry in Multiple Myeloma: Impact on Outcome in the Medical Research Council Myeloma IX Study. J Clin Oncol.

[65]Rayleigh, L. (1892). On the influence of obstacles arranged in rectangular order upon the properties of a medium. In Philosophical Magazine, pp. 481-502.

[66]Richards, A.L., Dickey, M.D., Kennedy, A.S., and Buckner, G.D. (2012). Design and demonstration of a novel micro-Coulter counter utilizing liquid metal electrodes. J Micromech Microeng 22.

[67]Roberts, K., Parameswaran, M., Moore, M., and Muller, R.S. (1999). A silicon microfabricated aperture for counting cells using the aperture impedance technique. Canadian Journal of Electrical and Computer Engineering-Revue Canadienne De Genie Electrique Et Informatique 24, 109-113.

[68]Rodriguez-Trujillo, R., Castillo-Fernandez, O., Garrido, M., Arundell, M., Valencia, A., and Gomila, G. (2008). High-speed particle detection in a micro-Coulter counter with two-dimensional adjustable aperture. Biosensors \& Bioelectronics 24, 290-296. 
[69]Rodriguez-Trujillo, R., Mills, C.A., Samitier, J., and Gomila, G. (2007). Low cost micro-Coulter counter with hydrodynamic focusing. Microfluid Nanofluid 3, 171176.

[70] Saleh, O. (2003). A novel resistive pulse sensor for biological measurements. (New Jersey, USA, Princeton University).

[71]Saleh, O.A., and Sohn, L.L. (2002). An Artificial Nanopore for Molecular Sensing. Nano letters 3, 37-38.

[72] Saleh, O.A., and Sohn, L.L. (2003). Direct detection of antibody-antigen binding using an on-chip artificial pore. Proc Natl Acad Sci U S A 100, 820-824.

[73]Sato, H., Sasamoto, Y., Yagyu, D., Sekiguchi, T., and Shoji, S. (2007). 3D sheath flow using hydrodynamic position control of the sample flow. J Micromech Microeng 17, 2211.

[74]Scott, R., Sethu, P., and Harnett, C.K. (2008a). Three-dimensional hydrodynamic focusing in a microfluidic Coulter counter. The Review of scientific instruments 79, 046104.

[75] Simonnet, C., and Groisman, A. (2006). High-throughput and high-resolution flow cytometry in molded microfluidic devices. Analytical chemistry 78, 5653-5663.

[76]Song, Y., Zhang, H., Chon, C.H., Chen, S., Pan, X., and Li, D. (2010). Counting bacteria on a microfluidic chip. Analytica chimica acta 681, 82-86.

[77]Spencer, D., and Morgan, H. (2011). Positional dependence of particles in microfludic impedance cytometry. Lab on a Chip 11, 1234-1239.

[78] Stober, G., Steinbock, L.J., and Keyser, U.F. (2009). Modeling of colloidal transport in capillaries. Journal of Applied Physics 105, 084702.

[79]Sun, T., Green, N.G., Gawad, S., and Morgan, H. (2007). Analytical electric field and sensitivity analysis for two microfluidic impedance cytometer designs. Iet Nanobiotechnology 1, 69-79. 
[80]Sun, T., and Morgan, H. (2010). Single-cell microfluidic impedance cytometry: a review. Microfluid Nanofluid 8, 423-443.

[81]Tan, K.H., Chua, C.K., Leong, K.F., Cheah, C.M., Cheang, P., Abu Bakar, M.S., and Cha, S.W. (2003). Scaffold development using selective laser sintering of polyetheretherketone-hydroxyapatite biocomposite blends. Biomaterials 24, 31153123.

[82]Testa, G., and Bernini, R. (2012). Micro flow cytometer with 3D hydrodynamic focusing. pp. $82120 \mathrm{H}-82120 \mathrm{H}-82127$.

[83]Todd, S., Stephen Q (2005). Microfluidics: Fluid physics at the nanoliter scale. Reviews of Modern Physics 77, 977-1026.

[84] Trefethen, L.N. (1980). Numerical Computation of the Schwarz-Christoffel Transformation. Siam Journal on Scientific and Statistical Computing 1, 82-102.

[85]Tsai, C.H., Hou, H.H., and Fu, L.M. (2008). An optimal three-dimensional focusing technique for micro-flow cytometers. Microfluid Nanofluid 5, 827-836.

[86]Tsutsui, H., and Ho, C.M. (2009). Cell Separation by Non-Inertial Force Fields in Microfluidic Systems. Mech Res Commun 36, 92-103.

[87]Uehara, S., Tsutsui, M., Doi, K., Taniguchi, M., Kawano, S., and Kawai, T. (2013). Fluid Dynamics and Electrical Detection of \&lambda;DNA in Electrode-Embedded Nanochannels. Journal of Biomechanical Science and Engineering 8, 244-256.

[88] Wang, F., Wang H, Wang J, Wang HY, Peter L (2008). Microfluidic delivery of small molecules into mammalian cells based on hydrodynamic focusing. Biotechnology and bioengineering 100, 150-158.

[89] Wang, Y.-q., Wang, J.-y., Chen, H.-1., Zhu, Z.-c., and Wang, B. (2012). Prototype of a novel micro-machined cytometer and its 3D hydrodynamic focusing properties. Microsystem Technologies 18, 1991-2001.

[90]White, F.M. (1991). Viscous Fluid Flow 2edn (University of Rhode Island: McGrawhill, Inc.). 
[91]Wolff, A., Perch-Nielsen, I.R., Larsen, U.D., Friis, P., Goranovic, G., Poulsen, C.R., Kutter, J.P., and Telleman, P. (2003). Integrating advanced functionality in a microfabricated high-throughput fluorescent-activated cell sorter. Lab on a chip 3, $22-27$.

[92]Wood, D.K., Requa, M.V., and Cleland, A.N. (2007). Microfabricated highthroughput electronic particle detector. Review of Scientific Instruments 78.

[93]Wu, Y., Benson, J.D., Critser, J.K., and Almasri, M. (2010). MEMS-based Coulter counter for cell counting and sizing using multiple electrodes. J Micromech Microeng 20.

[94]Wu, Y., Han, X., Benson, J.D., and Almasri, M. (2012). Micromachined Coulter counter for dynamic impedance study of time sensitive cells. Biomed Microdevices $14,739-750$.

[95]Wu, Z., Nguyen NT (2005). Hydrodynamic focusing in microchannels under consideration of diffusive dispersion: theories and experiments. Sensors and Actuators B 107, 965-974.

[96]Xiang, Q., Xuan, X., Xu, B., and Li, D. (2005). Multi - Functional Particle Detection with Embedded Optical Fibers in a Poly(dimethylsiloxane) Chip. Instrumentation Science \& Technology 33, 597-607.

[97]Yang, A.S., and Wu, C.Y. (2006). Computational design approach to hydrodynamic focusing in a flow cytometer. Lect Notes Comput Sc 4115, 336-341.

[98]Zhang, H.P., Chon, C.H., Pan, X.X., and Li, D.Q. (2009). Methods for counting particles in microfluidic applications. Microfluid Nanofluid 7, 739-749.

[99]Zhe, J., Jagtiani A, Dutta P, Carletta J (2007). A micromachined high throughput Coulter counter for bioparticle detection and counting. J Micromech Microeng 17, 304-313.

[100] Zhuang, G., Jensen TG, Kutter JP (2008). THREE-DIMENSIONAL HYDRODYNAMIC FOCUSING OVER A WIDE REYNOLDS NUMBER RANGE 


\section{CURRICULUM VITAE}

\section{Skills}

Numerical simulation: $\quad 3 \mathrm{D}$ tet/hexcore/prisms grid generation, boundary layer mesh refining, full/local grids repairing/improving/replacing, hybrid grid meshing, injection simulation.

Programming/Software Hypermesh, Abaqus, Tgrid, ANSYS/Fluent, Star-CD, tools: SolidWorks, Tecplot, Paraview, C/C++.

\section{Education}

University of Louisville, Louisville, KY

Expected June 2014

Ph.D. Mechanical Engineering (CFD)

Tongii University, Shanghai, China

March 2009

M.S. Mechanical Engineering (Mechatronics)

Tongji University, Shanghai, China

June 2006

B.S. Mechanical Engineering (Heat transfer/Refrigeration)

\section{Experiences}

\section{Caterpillar Inc. 7/13-present}

- CFD analysis on the NRS (NOx reduction system) for the after-treatment of engine

$>$ Simulated the liquid particle trajectory, vapor and temperature distribution. Optimized the mixer angle, size and position to improve the NOx mixing performance.

$>$ Modeled the steady/transient air flow velocity distribution and air recirculation phenomenon for the exhausted pipe. Studied the impact of the pre-mixer and optimize its angle, size and position. Also considered the variation of the pipe geometry/number. 


\section{University of Louisville, Louisville, KY USA 8/09-7/13}

- Signal modeling for a Lab-on-a-Chip microfluidic System (Micro Coulter counter)

$>$ Modeled the ion concentration distribution and tested the cell motion.

$>$ Provided the electrical field simulation and analyzed the signal sensitivity variation.

- Gas/liquid micro flow modeling by Fluent (Multiphase Flow Model)

$>$ Simulated two-phase phenomena (Drop-Ejection, Fuel Tank Sloshing) by Fluent

$>$ Modeled Taylor bubble flow by VOF, estimated the bubble gap.

\section{Tongji University, ShangHai China 10/06-03/09}

- Designed an image edge detection algorithm by Matlab and detected the image edges by Gauss operator.

-Designed an 8-Cylinders Synchronous Hydraulic System. Advanced Modeling Environment for Simulation of engineering systems (AMESim) was used to simulate the performance.

\section{Publications}

Zhang M, Lian Y, "Numerical Investigation of the Coulter Principle in a Microfluidic Device". ASME 2013 Fluids Engineering Summer Meeting

Zhang M, Lian Y, Harnett C, Brehob E, "Investigation of Hydrodynamic Focusing in a Microfluidic Coulter Counter Device". Journal of Biomechanical Engineering, v 134, n 8, p 081001 (9 pp.), Aug. 2012

\section{Awards}

Excellent Student Scholarship, Tongji University, 2002-2005

KSEF (Kentucky Science and Engineering Foundation) Award, KY, 2010- 2012

Fellowship, University of Louisville, 2009-2013 